\title{
0/1/all CSPs, Half-Integral $A$-path Packing, and Linear-Time FPT Algorithms
}

\author{
Yoichi Iwata* \\ National Institute of Informatics \\ yiwata@nii.ac.jp
}

\author{
Yutaro Yamaguchi ${ }^{\dagger}$ \\ Osaka University \\ yutaro_yamaguchi@ist.osaka-u.ac.jp
}

\author{
Yuichi Yoshida ${ }^{\ddagger}$ \\ National Institute of Informatics \\ yyoshida@nii.ac.jp
}

\begin{abstract}
A recent trend in the design of FPT algorithms is exploiting the half-integrality of LP relaxations. In other words, starting with a half-integral optimal solution to an LP relaxation, we assign integral values to variables one-by-one by branch and bound. This technique is general and the resulting time complexity has a low dependency on the parameter. However, the time complexity often becomes a large polynomial in the input size because we need to compute half-integral optimal LP solutions.

In this paper, we address this issue by providing an $O(\mathrm{~km})$-time algorithm for solving the LPs arising from various FPT problems, where $k$ is the optimal value and $m$ is the number of edges/constraints. Our algorithm is based on interesting connections among $0 / 1 /$ all constraints, which has been studied in the field of constraints satisfaction, $A$-path packing, which has been studied in the field of combinatorial optimization, and the LPs used in FPT algorithms. With the aid of this algorithm, we obtain improved FPT algorithms for various problems, including Group Feedback Vertex Set, Subset Feedback Vertex Set, Node Multiway Cut, Node Unique label Cover, and Non-monochromatic Cycle Transversal. The obtained running time for each of these problems is linear in the input size and has the current smallest dependency on the parameter. In particular, these algorithms are the first linear-time FPT algorithms for problems including Group Feedback Vertex Set and Nonmonochromatic Cycle Transversal.
\end{abstract}

${ }^{*}$ Supported by JSPS KAKENHI Grant Number JP17K12643.

${ }^{\dagger}$ Supported by JSPS KAKENHI Grant Number JP16H06931 and JST ACT-I Grant Number JPMJPR16UR.

${ }^{\ddagger}$ Supported by JST ERATO Grant Number JPMJER1305 and JSPS KAKENHI Grant Number JP17H04676. 


\section{Contents}

1 Introduction 1

1.1 FPT Algorithms using Half-Integral LP Relaxations . . . . . . . . . . . . . . . 1

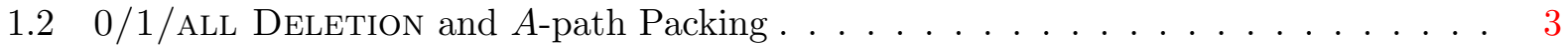

1.3 Related Work on Half-Integral $A$-path Packing . . . . . . . . . . . . . . . 4

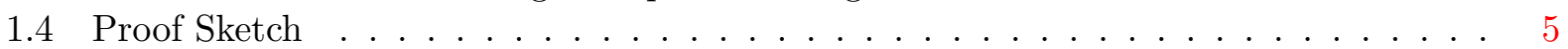

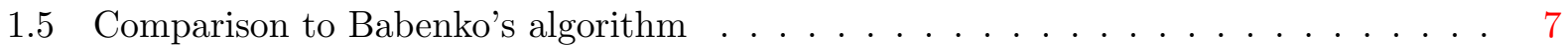

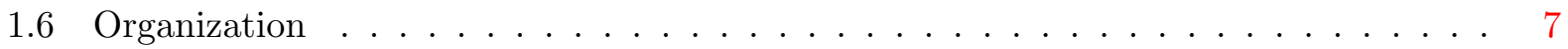

2 Definitions $\quad 8$

2.1 Basic Notations . . . . . . . . . . . . . . . . . . . . . 8

$2.20 / 1$ All Deletion and Half-Integral Relaxation . . . . . . . . . . . . . . 8

2.3 Single-Branching Pair and Incremental-Test Oracle . . . . . . . . . . . . . . . . 10

3 Half-Integral Packing and Covering 11

3.1 Preliminaries . . . . . . . . . . . . . . . . . . . . . 11

3.1 Basic $\mathcal{F}$-Packing . . . . . . . . . . . . . . . . . . 11

3.1 .2 Augmenting Path/Pair . . . . . . . . . . . . . . . . . . . . . . . . . . . . . . . . . .

3.2 Finding Augmenting Path/Pair . . . . . . . . . . . . . . . . . . . . . . . . . . . . . .

3.3 Constructing Half-Integral $\mathcal{F}$-Cover $\ldots \ldots \ldots \ldots \ldots$

3.4 Augmentation . . . . . . . . . . . . . . . . . . . . . 21

3.4.1 Simplification of Alternating Path . . . . . . . . . . . . . . 21

3.4.2 Augmentation by Augmenting Path . . . . . . . . . . . . . . . . . 23

3.4.3 Augmentation by Augmenting Pair . . . . . . . . . . . . . . 25

4 Farthest Cover $\quad 31$

5 Linear-Time FPT Algorithms

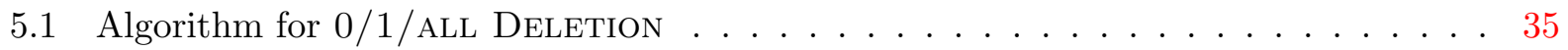

5.2 Applications to Other Problems . . . . . . . . . . . . . . . . . 39

$\begin{array}{ll}\text { A Proof of Persistency } & 46\end{array}$

$\begin{array}{ll}\text { B Axiomatic Model } & 47\end{array}$ 


\section{Introduction}

\subsection{FPT Algorithms using Half-Integral LP Relaxations}

Parameterized complexity is the subject of studying the complexity of parameterized problems. A parameterized problem with a parameter $k$ is fixed parameter tractable (FPT) if we can solve the problem in $f(k) \operatorname{poly}(n)$ time, where $n$ is the input size. Various parameterized problems are known to be FPT. See $[8,10]$ and references therein for a comprehensive list of FPT problems.

One of the motivations of studying parameterized complexity is to understand tractable subclasses of (NP-)hard problems; hence, the primary interest has been which parameterized problems admit FPT algorithms. However, from a practical point of view, the running time with respect to the input size must also be small. Indeed, linear-time FPT algorithms (i.e., FPT algorithms whose running times are linear in the input size) are proposed for several problems including Treewidth [4], Almost 2-SAT [18,31], Feedback Vertex Set (FVS) [3], Subset FVS [21], Directed FVS [22], and Node Unique Label Cover [23]. These works focused on reducing the running time with respect to the input size, and hence dependency on the parameter is often suboptimal. For example, Subset FVS admits an FPT algorithm running in $O^{*}\left(4^{k}\right)^{1}$ time [19] whereas the current best linear-time FPT algorithm has time complexity $O\left(25.6^{k} m\right)$ [21], where $m$ is the number of edges in the input graph.

The half-integrality of the LP relaxations has recently been used to design FPT algorithms for a broad range of problems $[9,15,17,19,20,33]$. To see the idea, let us consider a minimization problem whose goal is to find a solution of size $k$, and suppose that it admits a half-integral LP relaxation $^{2}$, that is, an LP relaxation with an optimal LP solution that only uses values in $\left\{0, \frac{1}{2}, 1\right\}$. The algorithm is based on the standard branch-and-bound framework. First, we compute a halfintegral (optimal) LP solution. We can stop if all the variables have integral values or the sum of the values in the LP solution exceeds $k$. Otherwise, we fix variables with values 1 . We then pick an arbitrary variable with value $\frac{1}{2}$, and branch into the case that its value is fixed to 0 and the case that its value is fixed to 1 . This approach has several big advantages. It can be applicable to various problems just by changing the LP, and has a small time complexity with respect to parameter $k$. For many problems including Almost 2-SAT [20], Node Multiway Cut [9], and GROUP FVS [19], the current smallest dependency on the parameter is indeed achieved by this approach.

A drawback of the abovementioned approach is that it is not trivial how to efficiently compute half-integral LP solutions. Iwata, Wahlström, and Yoshida [19] viewed half-integrality as a discrete relaxation. They showed that one can compute half-integral LP solutions for ALmost 2-SAT and (Edge) Unique Label Cover in time linear in the input size by reducing them to the $s$ - $t$ cut problem. Moreover, they showed that we can compute LP solutions with an extremal condition, which we call the farthest condition herein (see Section 2.2 for details), in the same running time. Using farthest solutions, the LP lower bound strictly increases for each branching. Consequently, they obtained linear-time FPT algorithms for these problems.

\footnotetext{
${ }^{1} O^{*}(\cdot)$ hides a polynomial dependency on the input size. When focusing on reducing the $f(k)$ part, the poly $(n)$ part is often ignored using this notation.

${ }^{2}$ Most of the LPs used in the FPT algorithms are not natural LP relaxations of the original problems, but are LP relaxations of rooted problems. For example, the rooted version of FVS is a problem of finding a minimum vertex subset $S$, such that the graph obtained by removing $S$ contains no cycles reachable from a prescribed vertex $s$. Note that the existence of a half-integral LP relaxation to the rooted problem does not imply a 2-approximability of the original problem.
} 
Table 1: Summary of our FPT results. Here, $d$ denotes the maximum domain size, $\Sigma$ is the alphabet set; $m$ denotes the number of edges/constraints in the input; and $T_{\Gamma}$ denotes the time complexity for performing group operations. All the algorithms are deterministic except for the $O\left(25.6^{k} m\right)$-time algorithm for SUBSET FVS. See Section 5.2 for the problem definitions.

\begin{tabular}{|c|c|c|c|}
\hline Problem & Smallest $f(k)$ & Existing linear-time FPT & Our result \\
\hline 0/1/ALL DELETION & $O^{*}\left(d^{2 k}\right)[19]$ & - & $O\left(d^{2 k} k m\right)$ \\
\hline Node Unique Label Cover & $O^{*}\left(|\Sigma|^{2 k}\right)[19]$ & $|\Sigma|^{O(k|\Sigma|)} m[23]$ & $O\left(|\Sigma|^{2 k} \mathrm{~km}\right)$ \\
\hline TWO-FAN DELETION & $O^{*}\left(9^{k}\right)[19]$ & - & $O\left(4^{k} k m\right)$ \\
\hline $\begin{array}{l}\text { MONOCHROMATICALLY } \\
\text { ORIENTABLE DELETION }\end{array}$ & - & - & $O\left(4^{k} k m\right)$ \\
\hline $\begin{array}{c}\text { SubSET PSEUDOFOREST } \\
\text { DELETION }\end{array}$ & - & - & $O\left(4^{k} k m\right)$ \\
\hline Node Multiway Cut & $O^{*}\left(2^{k}\right)[9]$ & $O\left(4^{k} m\right)[6,18]$ & $O\left(2^{k} \mathrm{~km}\right)$ \\
\hline GROUP FVS & $O^{*}\left(4^{k} T_{\Gamma}\right)[19]$ & - & $O\left(4^{k} k m T_{\Gamma}\right)$ \\
\hline Subset FVS & $O^{*}\left(4^{k}\right)[19]$ & $\begin{array}{c}O\left(25.6^{k} m\right) \text { (randomized) [21] } \\
2^{O(k \log k)} m \text { (deterministic) [21] }\end{array}$ & $O\left(4^{k} k m\right)$ \\
\hline $\begin{array}{c}\text { NON-MONOCHROMATIC } \\
\text { CYCLE TRANSVERSAL }\end{array}$ & $O^{*}\left(4^{k}\right)[33]$ & - & $O\left(4^{k} k m\right)$ \\
\hline
\end{tabular}

The $s$ - $t$ cut approach unfortunately does not work well for other problems such as Group FVS, Subset FVS, Node Multiway Cut, and Node Unique Label Cover because it is essentially applicable only to edge-deletion problems ${ }^{3}$ and because the auxiliary network size is generally not linear in the input size (e.g., for Subset Feedback Edge Set, the size of the network becomes $\left.2^{O(m)}\right)$. We need to resort to solving linear programs for these problems, and hence the resulting FPT algorithms have large dependencies on the input size. Among these problems, linear-time FPT algorithms have been obtained for Node Multiway Cut [6,18], Subset FVS [21], and Node Unique Label Cover [23] by problem-specific arguments without using LP relaxations. However, they have larger dependencies on $k$ (Table 1 ).

The main contribution of this study is the development of an algorithm that computes farthest half-integral solutions to those LPs in time linear in the input size. Using the connection of computing half-integral LP solutions and FPT algorithms, we obtain linear-time FPT algorithms for various problems, which are summarized in Table 1. In particular, for SubSET FVS, Node Multiway Cut, and Node Unique Label Cover, we substantially improve the dependency on the parameter. For the other problems including Group FVS and Non-monochromatic Cycle Transversal, we obtain the first linear-time FPT algorithms. We note that, for every problem in the table, the $f(k)$ part in the running time of our algorithm matches or improves the smallest known. All of these results are obtained by the same approach, i.e., the branch-and-bound framework combined with the efficient computation of half-integral LPs, which demonstrates its generality.

\footnotetext{
${ }^{3}$ Note that edge-deletion problems are easily reducible to the corresponding vertex-deletion problems in most cases by subdividing the edges and creating $k$ copies of the original vertices.
} 


\section{$1.2 \quad 0 / 1 /$ all Deletion and $A$-path Packing}

We consider the following problem, called 0/1/ALL DELETiOn, to establish a unified framework and provide linear-time FPT algorithms for the abovementioned problems using half-integral LP relaxations. Let $V$ be a set of variables. Each variable $v \in V$ has an individual domain $D(v)$. A function $\varphi$ on $V$ that maps each variable $v \in V$ to a value $\varphi(v) \in D(v)$ is called an assignment for $V$. We consider the following two types of binary constraints on $(u, v) \in V \times V$, called $0 / 1 /$ all constraints $[7]^{4}$.

1. Permutation constraint $\pi(\varphi(u))=\varphi(v)$, where $\pi: D(u) \rightarrow D(v)$ is a bijection.

2. Two-fan constraint $(\varphi(u)=a) \vee(\varphi(v)=b)$, where $a \in D(u)$ and $b \in D(v)$.

Let $C$ be a set of $0 / 1 /$ all constraints on $V$. We assume that $C$ contains at most one constraint for each pair of distinct variables $u, v \in V$. A constraint on $(u, v)$ in $C$ is denoted by $C_{u v}$. For a subset $U \subseteq V$, we denote the set $\left\{C_{u v} \in C \mid u, v \in U\right\}$ by $C[U]$.

0/1/All Deletion $\quad$ Parameter: $k, d$

Input: A set of variables $V$ each of which has a domain of size at most $d$, a set $C$ of $0 / 1$ /all constraints each of which is given as a table of size $O(d)$, a partial assignment $\varphi_{A}$ for a subset $A \subseteq V$, and an integer $k$.

Question: Is there a pair of a set $X \subseteq V$ of at most $k$ variables and a partial assignment $\varphi$ for $V \backslash X$ such that $(1) \varphi(v)=\varphi_{A}(v)$ holds for every $v \in A \backslash X$ and (2) $\varphi$ satisfies all of $C[V \backslash X]$ ?

The set $X$ in the question is called a deletion set. The task of the optimization version of this problem is to compute the size of a minimum deletion set. Various FPT problems can be expressed as $0 / 1$ Ald Deletion (by using a large $d$ ). Note that for several problems, we need exponentialsize domains, and hence a linear-time FPT algorithm for 0/1/ALL DELETION does not directly imply linear-time FPT algorithms for such problems. We show that, by giving constraints not as a table but as an oracle and by using a specialized branching strategy, we can obtain linear-time FPT algorithms even for such problems in a unified way. See Section 5.2 for details.

The primal graph for $C$ is a simple undirected graph $G=(V, E)$ such that an edge $u v \in E$ exists if and only if a constraint $C_{u v}$ on $(u, v)$ exists. An important property of the $0 / 1 /$ all constraints is that, when fixing the value of a variable $u \in V$ to $p \in D(u)$, the set of values of $v \in V$ satisfying the constraint $C_{u v}$ is either $D(v)$ (when the constraint is a two-fan with $a=p$ ) or a singleton $\{q\}$ (when the constraint is a permutation with $q=\pi(p)$ or a two-fan with $a \neq p$ and $b=q$ ). The latter-type implication is called a unit propagation.

When we are given a partial assignment $\varphi_{A}$ for a subset $A \subseteq V$, unit propagations occur along walks in the primal graph $G$ starting at the vertices in $A$. If the unit propagations along two different walks starting at (possibly the same) vertices in $A$ lead to a contradiction (i.e., implicate distinct singletons for the same variable), then at least one variable on the two walks must be contained in the deletion set. The concatenation of such two walks is a walk between the vertices in $A$ (called an $A$-walk) that is said to be conflicting (see Section 2.2 for a formal definition).

This observation provides a lower bound on the minimum size of a deletion set as follows. Suppose that a deletion set $X \subseteq V$ with $|X|=k$ exists, and let $\mathcal{F}=\mathcal{F}_{C, \varphi_{A}}$ be the set of all

\footnotetext{
${ }^{4}$ Precisely speaking, the permutation and two-fan constraints together with empty and complete constraints are obtained by enforcing arc-consistency on the $0 / 1$ /all constraints introduced in [7].
} 
conflicting $A$-walks in the primal graph $G$ for $C$ with respect to $\varphi_{A}$. Then, the remaining graph $G-X$ cannot have a walk in $\mathcal{F}$ (i.e., $X$ is a cover (or a hitting set) of $\mathcal{F}$ ). Hence, the minimum size of such a cover is at most $k$.

We now consider an LP relaxation of finding a minimum cover of the set $\mathcal{F}$ of all conflicting $A$-walks in $G$, called the $\mathcal{F}$-covering problem: we are required to find a function $x: V \rightarrow \mathbb{R}_{\geq 0}$ minimizing the total value $|x|:=\sum_{v \in V} x(v)$ under the constraint that $\sum_{v \in W} x(v) \geq 1$ for every $W \in \mathcal{F}$, where " $\sum_{v \in W}$ " means the summation over the occurrences of vertices $v$ in $W$ considering the multiplicity (e.g., $x(v)$ is summed twice if $W$ intersects $v$ twice). The dual of this LP is written down as follows, as the $\mathcal{F}$-packing problem: we are required to find a function $y: \mathcal{F} \rightarrow \mathbb{R}_{\geq 0}$ maximizing the total value $|y|:=\sum_{W \in \mathcal{F}} y(W)$ subject to $\sum_{W \in \mathcal{F}: v \in W} y(W) \leq 1$ for every $v \in V$, where we also consider the multiplicity of the occurrences of vertices in a walk in the summation

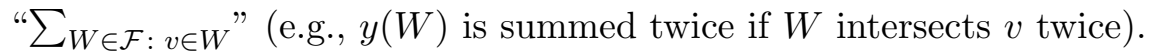

We then propose an $O(k m T)$-time algorithm for finding a pair of a half-integral $\mathcal{F}$-cover $x$ and a half-integral $\mathcal{F}$-packing $y$ with $|x|=|y| \leq \frac{k}{2}$ (if exists, and otherwise correctly concluding it), where $m=|C|$ and $T$ is the running time of a certain oracle for simulating unit propagations (see Section 2.3 for the detail). Note that, by LP duality, these $x$ and $y$ are both optimal solutions to the $\mathcal{F}$-covering and $\mathcal{F}$-packing problems, respectively.

Combining our algorithm with the result in [19] (see also Theorem 1 in Section 2.2), we can obtain an FPT algorithm for 0/1/ALL DELETION and, hence, FPT algorithms for other various problems. Section 5 provides the detailed discussion for each specific problem.

\subsection{Related Work on Half-Integral $A$-path Packing}

When $C$ contains only the permutation constraints, the integral version of the $\mathcal{F}$-packing problem has been studied under the name of non-returning A-path packing [26, 28,34]. This is the current most general case of tractable (integral) path packing problems. Our half-integral result suggests a conjecture that the integral packing of conflicting paths will also be tractable. We also believe that ideas behind our half-integral algorithm will be useful for obtaining a faster algorithm for integral path packing problems.

For a further special case (of non-returning $A$-path packing), called internally disjoint $t^{5} A$-path packing, the integral version of the dual covering problem coincides with NodE Multiway CuT, and its LP relaxation is used in the branch-and-bound FPT algorithm [9] and a 2-approximation algorithm [14] for this problem.

Several previous works in the field of combinatorial optimization can be found as regards the half-integral version of internally disjoint $A$-path packing. Garg et al. [14] and Pap [27, 29] found that both LPs always enjoy half-integral optimal solutions even if each non-terminal vertex has an individual integral capacity instead of 1. Hirai [16] and Pap [27, 29] developed algorithms for finding such half-integral optimal solutions, which both run in strongly polynomial time (i.e., the number of elementary operations performed through each algorithm does not depend on the capacity values). One makes use of a sophisticated algorithm for the submodular flow problem [13] whereas the other relies on the ellipsoid method to solve LPs whose coefficient matrices only have $0, \pm 1$ entries [12]. Specializing on the uncapacitated case, Babenko [1] provided an $O(k n m)$-time algorithm for finding a maximum half-integral packing, where $n$ and $m$ are the numbers of vertices and edges, respectively, and $k$ denotes the optimal value, which is at most $O(n)$. Our algorithm

\footnotetext{
${ }^{5}$ They can share terminals, but not inner vertex or edge.
} 

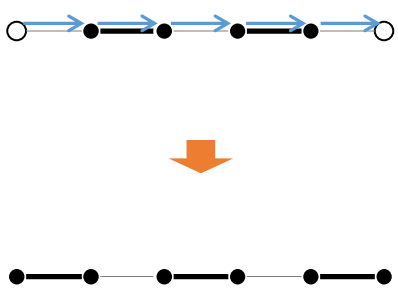

(1) connecting distinct vertices
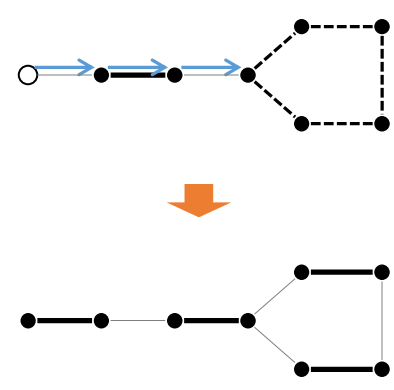

(2) path to an odd cycle
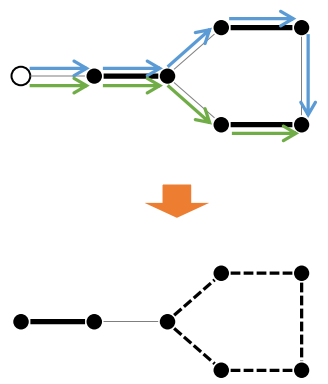

(3) prefix-sharing pair

Figure 1: Augmentations for half-integral matching. Thick solid lines denote edges of weight 1, dashed lines denote edges of weight $\frac{1}{2}$, and thin solid lines denote edges of weight 0

for the $\mathcal{F}$-packing/covering problems improves the previous best running time even against this (internally disjoint) special case.

\subsection{Proof Sketch}

Basically, we iteratively augment a half-integral $\mathcal{F}$-packing $y$, and construct a half-integral $\mathcal{F}$-cover $x$ of the same size when no augmentation is possible. Since the $\mathcal{F}$-covering and $\mathcal{F}$-packing problems are the dual LPs to each other, this implies that $x$ and $y$ are optimal solutions to these problems. In the case of the maximum $s$ - $t$ flow, when we failed to find an augmenting path, we can construct a minimum $s$ - $t$ cut by taking the set of edges on the flow that separates the vertices visited by the failed search from the unvisited vertices. Similarly, we can construct a minimum half-integral $\mathcal{F}$-cover by taking the set of vertices on the half-integral $\mathcal{F}$-packing that separates visited vertices from the unvisited vertices.

We describe the idea behind our algorithm for $\mathcal{F}$-packing by showing a relation to computing half-integral (non-bipartite) matchings, which are often called 2-matchings in the field of combinatorial optimization (see [32, Chapter 30] for the basics). Although we can easily obtain a maximum half-integral matching by a reduction to maximum bipartite matching [24], we propose a different approach herein. The idea behind this approach can be used for the half-integral $\mathcal{F}$-packing. We focus on special half-integral matchings that consist of vertex-disjoint edges of weight 1 and odd cycles $^{6}$ with each edge having weight $\frac{1}{2}$. A maximum half-integral matching with this special structure is known to always exist [2]. In each step, we search for an alternating path (a path that alternately uses edges of weight 0 and 1 and never uses the edges of weight $\frac{1}{2}$ ) from the vertices not used in the current matching. Augmentation can be categorized into three types (see Figure 1).

The first case is when we found an alternating path $P$ connecting two distinct vertices not used in the current matching. The current matching uses all the even edges in $P$. We can augment the matching size by 1 by taking all the odd edges and discarding all the even edges.

The second case is when we found an alternating path $P$ of length $2 a+1$ ending at a vertex on an odd cycle $C$ of length $2 b+1$. The current matching uses all the even edges in $P$ with weight 1 and all the edges in $C$ with weight $\frac{1}{2}$. Thus, the size of the current matching induced by $P$ and

\footnotetext{
${ }^{6}$ The number of edges in the cycle is odd.
} 
$C$ is $a+b+\frac{1}{2}$. We can easily augment this matching to an integral matching of size $a+b+1$ by alternately taking the edges in $P$ and $C$.

The third case is when we found a pair of prefix-sharing alternating paths $P$ of odd length and $Q$ of even length ending at the same vertex. This case corresponds to a blossom in Edmonds' algorithm for the maximum (integral) matching problem [11]. In Edmonds' algorithm, the alternation is applied to the common prefix and then the vertices on the cycles induced by $P$ and $Q$ are shrunk. In our approach, we can augment the matching size by $\frac{1}{2}$ by applying the alternation to the common prefix, then by transforming the cycle induced by $P$ and $Q$ to an odd cycle with each edge having weight $\frac{1}{2}$.

We use a similar approach in our algorithm for $\mathcal{F}$-packing/covering. We focus on a special type of half-integral $\mathcal{F}$-packings that consist of disjoint paths in $\mathcal{F}$ of weight 1 , called integral paths, and wheels, which are the sums of an odd number of walks in $\mathcal{F}$ of weight $\frac{1}{2}$ and correspond to odd cycles for matching. See Section 3.1.1 for the precise definition. Although a maximum half-integral packing with a similar special structure for a very special case of $\mathcal{F}$-packing, internally disjoint $A$ path packing, is known to always exist [25], the existence of such a special solution was previously unknown for any other cases. The correctness of our algorithm provides a constructive proof of the existence.

In each step, we search for an alternating path from the vertices in $A$ not used in the current packing. As opposed to the case of computing half-integral matchings, the alternating paths may use edges contained in wheels. However, for simplicity, we ignore such a case in the explanation that follows. Roughly speaking, an alternating path is a sequence of paths $P_{1}, \ldots, P_{\ell}$, where $P_{i}$ is a path internally disjoint from any integral path and wheel for odd $i$ and is fully contained in an integral path for even $i$. In the alternation operation, we replace the integral path containing $P_{2}$ with the path obtained by concatenating $P_{1}$ and the prefix of the integral path containing $P_{2}$. We then replace the integral path containing $P_{4}$ with ..., and so on. The definition of alternating paths is rather complicated in ensuring that each introduced path is in $\mathcal{F}$. However, it essentially plays the same role as that for the half-integral matching case.

Augmentation can be categorized into three types. Each of which corresponds to the one for the half-integral matching case. When we find an alternating path connecting two distinct vertices in $A$, we can augment the $\mathcal{F}$-packing by 1 by applying the alternation. When we find an alternating path ending at a vertex on a wheel that is the sum of $2 a+1$ walks in $\mathcal{F}$ of weight $\frac{1}{2}$, we can augment the $\mathcal{F}$-packing by $\frac{1}{2}$ by decomposing the wheel into $a$ integral paths and introducing a new integral path. We call these two types of alternating path as an augmenting path. When we find a pair of prefix-sharing alternating paths $P$ and $Q$ ending at the same vertex (with some additional conditions), we can augment the $\mathcal{F}$-packing by $\frac{1}{2}$ by applying the alternation to the common prefix and then by introducing a new wheel. We call this type of a pair of alternating paths as an augmenting pair.

We need to test the membership in $\mathcal{F}$ in constant time to achieve the linear time complexity. To this end, we exploit an observation that the algorithm only tests membership against some special walks.

To obtain linear-time FPT algorithms, we need to compute a farthest minimum half-integral $\mathcal{F}$-cover, which is an $\mathcal{F}$-cover satisfying some extremal condition, in linear time. In the existing work using the $s$ - $t$ cut approach [19], this is achieved by exploiting a structural property of all the minimum $s$ - $t$ cuts [30]. We use a different approach because we do not have the corresponding structural property for all the minimum half-integral $\mathcal{F}$-covers. Naively, we can find a farthest 
minimum half-integral $\mathcal{F}$-cover by at most $n$ computations of the minimum half-integral $\mathcal{F}$-cover. We show that we can regard the whole sequence of computations as at most $2 k$ computations of augmenting path/pair search by properly deciding the order of these computations using the maximum half-integral $\mathcal{F}$-packing. Thus, it runs in linear time in total.

Another obstacle for obtaining linear-time FPT algorithms is the existence of two-fan constraints. Because 2-SAT can be expressed as 0/1/All Deletion with $k=0$ and $d=2$, any linear-time FPT algorithm for 0/1/ALL DELETION must be able to solve 2-SAT in linear time. The standard linear-time algorithm for 2-SAT uses the strongly connected component decomposition of the implication graph, and the existing linear-time FPT algorithms for Almost 2-SAT, a parameterized version of 2-SAT, also use the strongly connected component decomposition of an auxiliary network $[18,19,31]$. We cannot use this approach because the size of the auxiliary network becomes super-linear for our problems. In our algorithm, we do not use the strongly connected component decomposition, but instead use a parallel unit-propagation, which is an alternative linear-time algorithm for 2-SAT.

\subsection{Comparison to Babenko's algorithm}

Because our algorithm for the $\mathcal{F}$-packing/covering problems improves the previous best running time even against the internally-disjoint special case, we compare our algorithm with Babenko's algorithm to clarify the reason that we obtain such an improvement. While both the algorithms iteratively augment a packing, the approaches are completely different. The main difference is the existence of augmenting pairs and the algorithm for computing augmenting paths/pairs.

In our algorithm, we focus on packings with a special structure. Both the definition of alternating paths and the algorithm for searching augmenting paths/pairs strongly rely on this structure. While the existence of a maximum half-integral packing with the special structure was already known for internally disjoint $A$-path packing [25], Babenko's algorithm does not directly exploit the structure, but uses a much weaker structure. This is because his augmentation strategy does not preserve the special structure because it does not consider a notion corresponding to augmenting pairs of our algorithm.

In our algorithm, we directly compute an augmenting path/pair in $O(m)$ time and hence we can compute a maximum packing of size $k$ in $O(k m)$ time. In contrast, in Babenko's algorithm, an auxiliary network and its $s$ - $t$ flow $f$ are constructed from the current packing, then an $f$-augmenting path for the standard maximum flow problem is computed. From the obtained $f$-augmenting path, we can either augment the current packing or we can find a set of vertices that can be safely contracted to some vertex in $A$. Because contractions occur at most $O(n)$ time per augmentation, the running time becomes $O(k n m)$.

\subsection{Organization}

We introduce the notions used throughout the paper in Section 2. Section 3 shows a fast algorithm that computes a maximum half-integral $\mathcal{F}$-packing and transforms it into a minimum half-integral $\mathcal{F}$-cover. Section 4 presents a fast algorithm for computing a farthest minimum half-integral $\mathcal{F}$ cover. We provide linear-time FPT algorithms in Section 5 using this algorithm. 


\section{Definitions}

\section{$2.1 \quad$ Basic Notations}

The multiplicity function $\mathbf{1}_{S}: U \rightarrow \mathbb{Z}_{\geq 0}$ for a multiset $S$ on the ground set $U$ is defined such that $\mathbf{1}_{S}(a)$ is the number of times that $a \in U$ appears in $S$. For two multisets $A$ and $B$ on the same ground set $U$, we denote by $A \backslash B$ the multiset such that $\mathbf{1}_{A \backslash B}(a)=\max \left\{\mathbf{1}_{A}(a)-\mathbf{1}_{B}(a), 0\right\}$ holds for any element $a \in U$. For a function $f: U \rightarrow \mathbb{R}$ and a multiset $S$ on the ground set $U$, we define $f(S):=\sum_{a \in U} \mathbf{1}_{S}(a) f(a)$. For a value $i \in \mathbb{R}$, we define $f^{-1}(i):=\{a \in U \mid f(a)=i\}$.

All the graphs in this study are undirected. However, we sometimes need to take care of the direction of edges. For an undirected graph $G=(V, E)$, we use the symbol $\hat{E}$ when we take care of the direction of the edges, i.e., $u v=v u$ for $u v \in E$ but $u v \neq v u$ for $u v \in \hat{E}$. For simplicity, we assume that the graphs are simple; if a graph contains multiple edges or self-loops, we can easily obtain an equivalent simple graph by subdividing the edges. For vertex $v \in V$, we denote the set of incident edges by $\delta(v)$. For a subset $U \subseteq V$, we denote the induced subgraph by $G[U]=(U, E[U])$.

For an undirected graph $G=(V, E)$, we define a walk in $G$ as an ordered list $W=\left(v_{0}, \ldots, v_{\ell}\right)$ of vertices such that $v_{i-1} v_{i} \in E$ for all $i=1, \ldots, \ell$. The integer $\ell$ is called the length of the walk. We denote the first and last vertices of $W$ by $s(W)=v_{0}$ and by $t(W)=v_{\ell}$, respectively, and we say that $W$ starts from $s(W)$ and ends at $t(W)$. We denote the multisets of vertices, inner vertices, and (undirected) edges appeared in $W$ by $V(W)=\left\{v_{0}, \ldots, v_{\ell}\right\}$, by $V_{\text {in }}(W)=\left\{v_{1}, \ldots, v_{\ell-1}\right\}=$ $V(W) \backslash\{s(W), t(W)\}$, and by $E(W)=\left\{v_{0} v_{1}, \ldots, v_{\ell-1} v_{\ell}\right\}$, respectively. For an edge $e=u v \in \hat{E}$, we simply use the same symbol $e$ to denote the walk $(u, v)$. A walk $W$ is called a (simple) path if $\mathbf{1}_{V(W)}(v) \leq 1$ for every $v \in V$. A walk $W$ is called a closed walk if $s(W)=t(W)$. A closed walk $W$ is called a (simple) cycle if $\ell \geq 3$ and $\mathbf{1}_{V(W) \backslash\{s(W)\}}(v) \leq 1$ for every $v \in V$ (which implies $\mathbf{1}_{V(W)}(s(W))=2$ ). We may regard a walk $W$ as a subgraph by ignoring the direction and the multiplicity. We say that a walk $W$ is internally disjoint from a subgraph $G^{\prime}=\left(V^{\prime}, E^{\prime}\right)$ if none of the inner vertices of $W$ are in $V^{\prime}$ and none of the edges of $W$ are in $E^{\prime}$. For a walk $W=\left(v_{0}, \ldots, v_{\ell}\right)$, we define the reversed walk as $W^{-1}=\left(v_{\ell}, \ldots, v_{0}\right)$. For a walk $W_{1}=\left(v_{0}, \ldots, v_{\ell^{\prime}}\right)$ and a walk $W_{2}=\left(v_{\ell^{\prime}}, \ldots, v_{\ell}\right)$ (where $0 \leq \ell^{\prime} \leq \ell$ ), we define the concatenation of the two walks as $W_{1} \circ W_{2}=\left(v_{0}, \ldots, v_{\ell}\right)$. The notation $W_{1} \circ W_{2}$ implicitly implies $t\left(W_{1}\right)=s\left(W_{2}\right)$.

\subsection{0/1/all Deletion and Half-Integral Relaxation}

We first recall 0/1/ALL DeLETION defined in Section 1.2. We are given a set $V$ of variables $v$ with individual domains $D(v)$, a set $C$ of $0 / 1 /$ all constraints (permutation and two-fan constraints) that can be represented by a simple undirected graph $G$, called the primal graph, and a partial assignment $\varphi_{A}$ for a subset $A \subseteq V$. The task is to determine whether a deletion set $X \subseteq V$ of at most $k$ variables exists, for which there exists an assignment $\varphi$ for $V \backslash X$ such that (1) $\varphi(v)=\varphi_{A}(v)$ holds for every $v \in A \backslash X$, and (2) $\varphi$ satisfies every constraint $C_{u v} \in C[V \backslash X]$.

As described in Section 1.2, an important property of the 0/1/all constraints is that, when fixing the value of a variable $u \in V$ to $p \in D(u)$, the set of values of $v \in V$ satisfying the constraint $C_{u v}$ is either $D(v)$ or a singleton $\{q\}$. We define $C_{u v}(p):=$ all in the former case and define $C_{u v}(p):=q$ in the latter case. We extend this definition to walks in the primal graph as follows. For a walk $(s)$ of length zero and an element $p \in D(s)$, we define $C_{(s)}(p):=p$. For a walk $W=W^{\prime} \circ e$ starting from $s \in V$ and an element $p \in D(s)$, we define $C_{W}(p):=$ all when $C_{W^{\prime}}(p)=$ all and $C_{W}(p):=C_{e}\left(C_{W^{\prime}}(p)\right)$ when $C_{W^{\prime}}(p) \neq$ all. Suppose that an assignment $\varphi$ satisfying all the 
constraints exists. Then, either $C_{W}(\varphi(s(W)))=$ all or $\varphi(t(W))=C_{W}(\varphi(s(W)))$ holds for any walk $W$ in the primal graph.

Let $\varphi_{A}$ be a partial assignment for a subset $A \subseteq V$. For a walk $W$ with $s(W) \in A$, we define $\operatorname{imp}_{\varphi_{A}}(W):=C_{W}\left(\varphi_{A}(s(W))\right)$, which represents the set of assignments for $t(W)$ induced by $W$. A walk $W$ is called a $\varphi_{A}$-implicational walk if $s(W) \in A$ and $\operatorname{imp}_{\varphi_{A}}(W) \neq$ all. A $\varphi_{A}$-implicational walk $W$ is called $\varphi_{A}$-conflicting if $t(W) \in A$ and $\operatorname{imp}_{\varphi_{A}}(W) \neq \varphi_{A}(t(W))$ hold. We omit the prefix/subscript $\varphi_{A}$ if it is clear from the context. We use the following lemma.

Lemma 1. For any two $\varphi_{A}$-implicational walks $P$ and $Q$ ending at the same vertex, $P \circ Q^{-1}$ is $\varphi_{A}$-conflicting if and only if $\operatorname{imp}_{\varphi_{A}}(P) \neq \operatorname{imp}_{\varphi_{A}}(Q)$.

Proof. We prove the lemma by induction on the length of $Q$. When the length of $Q$ is zero, $P \circ Q^{-1}=P$ is conflicting if and only if $\operatorname{imp}(P) \neq \varphi_{A}(t(P))=\operatorname{imp}(Q)$. When $Q=Q^{\prime} \circ v u$, we consider three cases.

(Case 1) If $P \circ u v$ is not implicational, $C_{u v}$ must be a two-fan $(\varphi(u)=a) \vee(\varphi(v)=b)$ and $\operatorname{imp}(P)=a$. Because $Q$ is implicational, we have $\operatorname{imp}(Q)=a$. Therefore, we have $\operatorname{imp}(P)=$ $\operatorname{imp}(Q)$. Because any prefix of a conflicting walk is implicational, $P \circ Q^{-1}$ is not conflicting. Thus, the lemma holds for this case.

(Case 2) If $P \circ u v$ is implicational and $C_{u v}$ is a permutation $\pi(\varphi(u))=\varphi(v)$, we have $\operatorname{imp}(P \circ$ $u v)=\pi(\operatorname{imp}(P))$ and $\operatorname{imp}\left(Q^{\prime}\right)=\pi(\operatorname{imp}(Q))$. Therefore, $\operatorname{imp}(P \circ u v)=\operatorname{imp}\left(Q^{\prime}\right)$ if and only if $\operatorname{imp}(P)=\operatorname{imp}(Q)$ holds. Thus, from the induction hypothesis, the lemma holds for this case.

(Case 3) If $P \circ u v$ is implicational and $C_{u v}$ is a two-fan $(\varphi(u)=a) \vee(\varphi(v)=b)$, we have $\operatorname{imp}(P) \neq a, \operatorname{imp}(Q)=a, \operatorname{imp}(P \circ u v)=b$, and $\operatorname{imp}\left(Q^{\prime}\right) \neq b$. Therefore, from the induction hypothesis, $P \circ Q^{-1}$ is conflicting. Thus, the lemma holds for this case.

For two walks $P$ and $Q$ ending at the same vertex, we write $P \not \equiv Q$ if $P \circ Q^{-1}$ is conflicting and $P \equiv Q$ if $P \circ Q^{-1}$ is not conflicting, but both $P$ and $Q$ are implicational ${ }^{7}$. The abovementioned lemma implies that $(\equiv)$ is an equivalence relation. This is a key property in our algorithms. Appendix B presents an alternative axiomatic definition of implicational/conflicting walks and show that any path systems admitting this key property can be expressed as the set of implicational/conflicting walks with $0 / 1$ /all constraints. We obtain the following corollaries from Lemma 1.

Corollary 1. If a walk $W$ is conflicting, then $W^{-1}$ is also conflicting.

Corollary 2. For three implicational walks $P, Q$, and $R$ ending at the same vertex, if $P \circ Q^{-1}$ is conflicting, then at least one of $P \circ R^{-1}$ and $R \circ Q^{-1}$ is conflicting.

We now introduce a half-integral relaxation ${ }^{8}$ of $0 / 1 /$ ALL DeLETION. Let $\mathcal{F}_{C, \varphi_{A}}$ denote the set of all $\varphi_{A}$-conflicting walks whose internal vertices do not intersect with $A^{9}$. When $C$ and $\varphi_{A}$ are clear from the context, we simply write $\mathcal{F}$ to refer to $\mathcal{F}_{C, \varphi_{A}}$. Note that from Corollary 1, we can ignore the direction of walks in $\mathcal{F}$. A function $x: V \rightarrow\left\{0, \frac{1}{2}, 1\right\}$ is called a half-integral $\mathcal{F}$-cover if $x(V(W)) \geq 1$ for every $W \in \mathcal{F}$. The size of $x$ is defined as $|x|=x(V)$. A function $y: \mathcal{F} \rightarrow\left\{0, \frac{1}{2}, 1\right\}$

\footnotetext{
${ }^{7}$ When at least one of $P$ or $Q$ is not implicational, neither $P \equiv Q$ nor $P \not \equiv Q$ holds.

${ }^{8}$ Originally we should define this as an LP relaxation, but we discuss it by assuming its half integrality, which is proved via our algorithm and LP duality.

${ }^{9}$ This constraint is only for simplicity. From Corollary 2, if $P \circ Q^{-1}$ is conflicting for some walks $P$ and $Q$ ending in $A$, at least one of $P$ and $Q$ is conflicting. Therefore, a maximum packing of conflicting walks that uses none of such walks always exists.
} 
is called a half-integral $\mathcal{F}$-packing if for every vertex $v \in V$, it holds that $\sum_{W \in \mathcal{F}} \mathbf{1}_{V(W)}(v) y(W) \leq 1$. The size of $y$ is defined as $|y|=y(\mathcal{F})$. From the LP-duality, we have $|x| \geq|y|$ for any pair of halfintegral $\mathcal{F}$-cover $x$ and half-integral $\mathcal{F}$-packing $y$. Any deletion set $X$ for $0 / 1 /$ ALL DeLetion must intersect every $\varphi_{A}$-conflicting walk; hence $\mathbf{1}_{X}$ is an integral $\mathcal{F}$-cover ${ }^{10}$. Therefore, the size of the minimum half-integral $\mathcal{F}$-cover provides a lower bound on the size of the minimum deletion set. For a half-integral $\mathcal{F}$-cover $x$, let $R(x)$ denote the set of vertices $t$ such that an implicational walk $W$ with $x(V(W))=0$ ending at $t$ exists.

We can prove the following property called persistency by a careful consideration of the results in [19] (see Appendix A for a detailed discussion).

Theorem 1. Let $C$ be a set of $0 / 1 /$ all constraints on a variable set $V$ and $\varphi_{A}$ be a partial assignment for a subset $A \subseteq V$. For any minimum half-integral $\mathcal{F}_{C, \varphi_{A}}$-cover $x$, there exists a minimum deletion set $X$ containing every vertex $u$ with $x(u)=1$ but avoiding every vertex in $R(x)$.

We say that a minimum half-integral $\mathcal{F}$-cover $x^{\prime}$ dominates a minimum half-integral $\mathcal{F}$-cover $x$ if $R(x) \subsetneq R\left(x^{\prime}\right)$ holds. A minimum half-integral $\mathcal{F}$-cover $x$ is called farthest if there exists no minimum half-integral $\mathcal{F}$-cover dominating $x$. Suppose that we have an $O(T)$-time algorithm for computing a farthest minimum half-integral $\mathcal{F}$-cover. From Theorem 1, it is not difficult to obtain an $O\left(d^{2 k} T\right)$-time FPT algorithm for 0/1/ALL DELETION. Moreover, the base $d$ of the exponent can be improved to a constant for several special cases. We give a detailed discussion in Section 5.1.

\subsection{Single-Branching Pair and Incremental-Test Oracle}

As shown in Section 5.2, various important NP-hard problems can be expressed as a special case of $0 / 1$ All Deletion. However, the domain size $d$ is often $\omega(1)$ (or even $\exp (m)$ for several cases, where $m$ is the number of edges/constraints). Hence, if every constraint is given as the table of size $d$, the total size of these tables already becomes super-linear. For obtaining linear-time FPT algorithms, we use oracles instead of the explicit expression of the constraints to efficiently check whether a given walk is implicational/conflicting or not.

A pair $(P, Q)$ of implicational walks ending at the same vertex is called single-branching if either $P \circ Q^{-1}$ forms a simple path or they can be written as $P=R \circ P^{\prime}$ and $Q=R \circ Q^{\prime}$ for a (possibly zero-length) path $R$ and two walks $P^{\prime}$ and $Q^{\prime}$ for which $P^{\prime} \circ Q^{\prime-1}$ forms a simple cycle that is internally disjoint from $R$. In our algorithm, we need to test whether a given walk is implicational and whether $P \circ Q^{-1}$ is conflicting for a given single-branching pair $(P, Q)$. To efficiently answer these queries, we use a tuple $(U, \mathcal{I}, \mathcal{A}, \mathcal{T})$, called an incremental-test oracle, of a set $U$ and functions $\mathcal{I}, \mathcal{A}$, and $\mathcal{T}$ satisfying the following.

- Init $\mathcal{I}: A \rightarrow U$.

- Append $\mathcal{A}: U \times \hat{E} \rightarrow U \cup\{$ all $\}$. Let $\mathcal{A}^{*}$ be a function such that

$$
\mathcal{A}^{*}((s))=\left\{\begin{array}{ll}
\mathcal{I}(s) & (s \in A) \\
\text { all } & (s \notin A)
\end{array} \quad \text { and } \quad \mathcal{A}^{*}(W \circ e)=\left\{\begin{array}{ll}
\mathcal{A}\left(\mathcal{A}^{*}(W), e\right) & \left(\mathcal{A}^{*}(W) \neq \text { all }\right) \\
\text { all } & \left(\mathcal{A}^{*}(W)=\text { all }\right)
\end{array} .\right.\right.
$$

Then, for any walk $W, \mathcal{A}^{*}(W) \neq$ all if and only if $W$ is implicational.

\footnotetext{
${ }^{10}$ Note that the converse may not hold. For example, we have $\mathcal{F}=\emptyset$ when $A=\emptyset$. Therefore, $X=\emptyset$ is the minimum integral $\mathcal{F}$-cover. In contrast, $X=\emptyset$ may not be a deletion set. Thus, the half-integral relaxation does not always lead to a 2-approximation algorithm.
} 
- Test $\mathcal{T}: U \times U \rightarrow\{$ true, false $\}$. For any single-branching pair $(P, Q), \mathcal{T}\left(\mathcal{A}^{*}(P), \mathcal{A}^{*}(Q)\right)=$ true if and only if $P \circ Q^{-1}$ is conflicting.

The running time of the incremental-test oracle is defined as the maximum running time of the three functions. In general, we can naively implement the incremental-test oracle by setting $U:=\bigcup_{u \in V} D(u), \mathcal{I}(s):=\varphi_{A}(s), \mathcal{A}(x, e)=C_{e}(x)$, and $\mathcal{T}(x, y):=$ true iff $x \neq y$. We can obtain a constant-time oracle by this naive implementation for several cases including NodE MulTiWAY Cut. Meanwhile, the naive implementation takes $O(m)$ time for several other cases including Subset Feedback Vertex Set. We will see in Section 5.2 that we can implement a constanttime oracle for these cases by exploiting the constraint that the inputs to the test function are restrited to single-branching pairs.

\section{$3 \quad$ Half-Integral Packing and Covering}

We prove the following theorem in this section.

Theorem 2. Let $C$ be a set of $0 / 1 /$ all constraints on variables $V$ and $\varphi_{A}$ be a partial assignment for a subset $A \subseteq V$. Given the primal graph of $C$, the set $A$, an incremental-test oracle for $\left(C, \varphi_{A}\right)$, and an integer $k$, we can compute a pair of minimum half-integral $\mathcal{F}_{C, \varphi_{A}}$-cover $x$ and maximum half-integral $\mathcal{F}_{C, \varphi_{A}}$-packing y with $|x|=|y| \leq \frac{k}{2}$ or correctly conclude that the size of the minimum half-integral $\mathcal{F}_{C, \varphi_{A}}$-cover is at least $\frac{k+1}{2}$ in $O(k m T)$ time, where $m$ is the number of constraints and $T$ is the running time of the incremental-test oracle.

Our algorithm is based on a simple augmentation strategy summarized as follows. Starting with $y(W)=0$ for every $W \in \mathcal{F}$, we repeatedly update a half-integral $\mathcal{F}$-packing $y$ that always consists of only two types of conflicting walks defined in Section 3.1.1. We search an augmenting path/pair (see Section 3.1.2) in each iteration using Algorithm 1 described in Section 3.2. If one is found, we can improve the current $\mathcal{F}$-packing $y$ in linear time by Lemmas 2 and 3 (Augmentation); otherwise, as shown in Section 3.3, we can naturally construct a half-integral $\mathcal{F}$-cover of size $|y|$, which guarantees the optimality of $y$ with the aid of the LP-duality. Since each augmentation increases $|y|$ by at least $\frac{1}{2}$, the number of iterations is bounded by $k+1$. Algorithm 1 can be implemented in linear time by Lemma 6, which concludes Theorem 2.

\subsection{Preliminaries}

\subsubsection{Basic $\mathcal{F}$-Packing}

In what follows, we focus on $\mathcal{F}$-packings that consist of only two types of conflicting walks. One is a simple path $I \in \mathcal{F}$ of weight 1 , which is called an integral path. The other is a wheel defined as follows and consists of an odd number of conflicting walks of weight $\frac{1}{2}$.

Definition 1. A pair of a simple cycle $C=H_{1} \circ \ldots \circ H_{d}$ of weight $\frac{1}{2}$ and (possibly zero-length) paths $\left\{S_{1}, \ldots, S_{d}\right\}$ of weight 1 is called a wheel if it satisfies the following conditions (Figure 2).

1. $d$ is an odd positive integer.

2. For any $i, S_{i}$ is a path from $A$ to $s\left(H_{i}\right)=t\left(H_{i-1}\right)$ (where $H_{0}=H_{d}$ ) that is internally disjoint from $C$. 


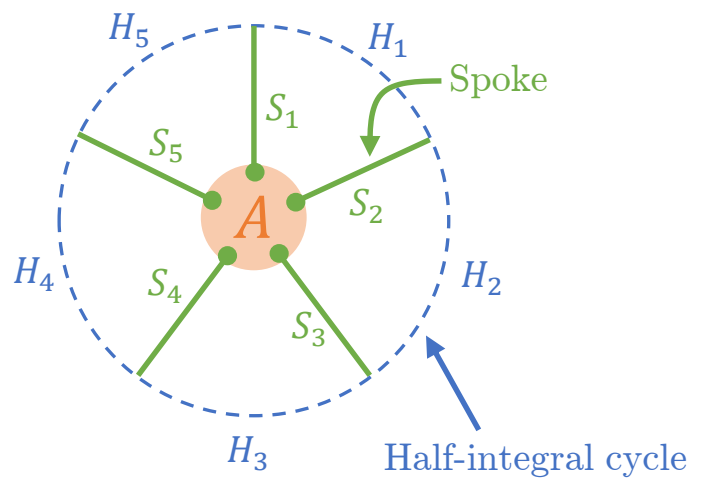

Figure 2: Wheel of degree 5.

3. For any distinct $i$ and $j, S_{i}$ and $S_{j}$ share no vertices.

4. For any $i, S_{i} \circ H_{i} \circ S_{i+1}^{-1} \in \mathcal{F}$, where $S_{d+1}=S_{1}$.

The integer $d$ is called the degree of the wheel, the cycle $C$ is called the half-integral cycle of the wheel, and the paths $\left\{S_{1}, \ldots, S_{d}\right\}$ are called the spokes of the wheel.

Note that a wheel of degree 1 is a closed walk $S_{1} \circ C \circ S_{1}^{-1} \in \mathcal{F}$ of weight $\frac{1}{2}$, and a wheel of degree $d \geq 3$ is a sum of $d$ simple paths $\left\{S_{1} \circ H_{1} \circ S_{2}^{-1}, \ldots, S_{d} \circ H_{d} \circ S_{1}^{-1}\right\} \subseteq \mathcal{F}$ of weight $\frac{1}{2}$.

A half-integral $\mathcal{F}$-packing $y$ is called a basic $\mathcal{F}$-packing if it is a sum of integral paths and wheels such that each vertex is contained in at most one of the integral paths and the wheels. In our algorithm, a basic $\mathcal{F}$-packing $y$ is dealt with as a weighted graph ${ }^{11}$ so that we can efficiently update integral paths and wheels in $y$. We denote by $V(y)$ and $E(y)$ the sets of vertices and (undirected) edges, respectively, that are contained in some integral path or wheel in $y$ (i.e., of positive weights), and particularly by $V_{1}(y)$ and $E_{1}(y)$, the sets of those contained in some integral path or spoke in $y$ (i.e., are of weight 1 ). A walk is called internally disjoint from $y$ if it is internally disjoint from the subgraph $(V(y), E(y))$.

For a basic $\mathcal{F}$-packing $y$, we define two functions $F_{y}$ (Forward) and $B_{y}$ (Backward) as follows (Figure 3). Let $P$ be a positive-length path contained in an integral path $I$ in $y$. From Corollary 1 , we can assume that $I$ has the same direction as $P$. We then define paths $F_{y}(P)$ and $B_{y}(P)$ such that $I=F_{y}(P) \circ P \circ B_{y}(P)^{-1}$ holds. For a vertex $v$ contained in a spoke $S$ in $y$, we denote by $F_{y}(v)$ the path from $t(S)$ to $v$ along $S$ and by $B_{y}(v)$ the path from $s(S)$ to $v$ along $S$ (i.e., $\left.F_{y}(v) \circ B_{y}(v)^{-1}=S^{-1}\right)$. For a path $P$ contained in a spoke $S$ in $y$ in the opposite direction to $S$, we define $F_{y}(P):=F_{y}(s(P))$ and $B_{y}(P):=B_{y}(t(P))$ (i.e., $F_{y}(P) \circ P \circ B_{y}(P)^{-1}=S^{-1}$ ). We omit the subscript $y$ if it is clear from the context.

\subsubsection{Augmenting Path/Pair}

We first define an alternating path to define our augmenting path/pair.

Definition 2. For a basic $\mathcal{F}$-packing $y$, a concatenation of paths $P=P_{1} \circ \cdots \circ P_{p}$ is called a $y$-alternating path if it satisfies all the following conditions.

\footnotetext{
${ }^{11}$ The weight is naturally defined for each vertex $v$ and edge $e$ by $\sum_{W \in \mathcal{F}} \mathbf{1}_{V(W)}(v) y(W)$ and $\sum_{W \in \mathcal{F}} \mathbf{1}_{E(W)}(e) y(W)$, respectively.
} 


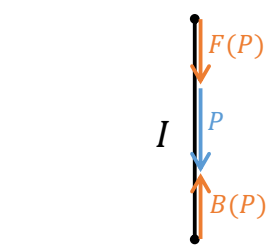

$P$ is contained in an integral path $I$

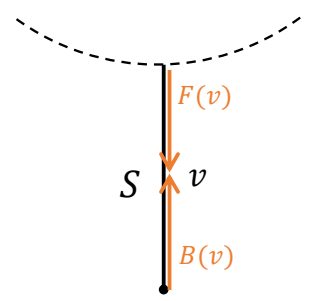

$v$ is contained in a spoke $S$

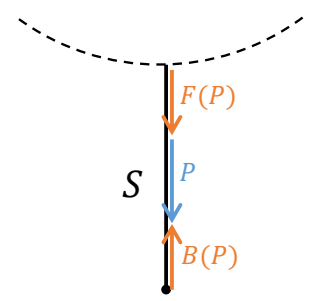

$P$ is contained in a spoke $S$

Figure 3: Definition of $F_{y}$ and $B_{y}$.

1. The edges in $E(P)$ are distinct (i.e., $\mathbf{1}_{E(P)}(e) \leq 1$ for every $\left.e \in E\right)$.

2. Every vertex in $P$ that is not contained in any integral path or spoke in $y$ appears in $P$ at most once $^{12}$ (i.e., $\mathbf{1}_{V(P)}(v) \leq 1$ for every $v \in V \backslash V_{1}(y)$ ).

3. $s(P) \in A \backslash V(y)$.

4. Each $P_{i}$ is a path of positive length satisfying the following conditions.

(a) For any odd $i, P_{i}$ is internally disjoint from $y$, and no internal vertex of $P_{i}$ is in $A$.

(b) For any even $i, P_{i}$ is contained in an integral path or a spoke in $y$. In the latter case, $P_{i}$ has the opposite direction to the spoke.

5. Let us define $B\left(P_{0}\right):=(s(P))$. The following conditions are satisfied for any $i^{13}$ (see Figure 4$)$.

(a) If $i$ is odd, $B\left(P_{i-1}\right) \circ P_{i}$ is implicational.

(b) If $i$ is even and $P_{i}$ is contained in an integral path, then $B\left(P_{i-2}\right) \circ P_{i-1} \not \equiv F\left(P_{i}\right)$, and none of the $P_{j}$ 's are contained in $B\left(P_{i}\right)$ for $j>i$. Moreover, if $B\left(P_{i-2}\right) \circ P_{i-1} \not \equiv B\left(P_{i}\right) \circ P_{i}^{-1}$, none of the $P_{j}$ 's are contained in $F\left(P_{i}\right)$ for $j>i$.

(c) If $i$ is even and $P_{i}$ is contained in a spoke, then $B\left(P_{i-2}\right) \circ P_{i-1} \equiv B\left(P_{i}\right) \circ P_{i}^{-1}$, and none of the $P_{j}$ 's are contained in $B\left(P_{i}\right)$ for $j>i$.

Each $P_{i}$ is called a segment of $P$. If $P$ consists of only a single segment, it is called single-segment. A zero-length path $P=(s)$ with $s \in A \backslash V(y)$ is considered as a $y$-alternating path with zero segments.

We define $T_{y}(P)$ (Tail) for a $y$-alternating path $P=P_{1} \circ \cdots \circ P_{p}$ as follows: $T_{y}(P):=B_{y}\left(P_{p-1}\right) \circ$ $P_{p}$ if $p$ is odd, and $T_{y}(P):=B_{y}\left(P_{p}\right)$ if $p$ is even. Note that $T_{y}(P)$ is always implicational. We omit the subscript $y$ if it is clear from the context.

Definition 3. A $y$-alternating path $P=P_{1} \circ \ldots \circ P_{p}$ is called a $y$-augmenting path if $p$ is odd and one of the following conditions is satisfied.

\footnotetext{
${ }^{12}$ From the other properties of the $y$-alternating paths, a vertex contained in an integral path or a spoke can appear twice in $P$. Hence, $P$ may not be a path in the precise sense.

${ }^{13}$ Technically, the conditions "none of the $P_{j}$ 's are contained in ..." mean that $P$ does not admit a shortcut (e.g., if there is some $P_{j}$ with $j>i$ contained in $B\left(P_{i}\right)$, we can obtain another $y$-alternating path $P_{1} \circ \cdots \circ P_{i-1} \circ W \circ P_{j+1} \circ \cdots P_{p}$, where $W$ is the path from $s\left(P_{i}\right)$ to $t\left(P_{j}\right)$ along the integral path or spoke). As in the case of matroid intersection, we need a shortcut-less alternating path.
} 


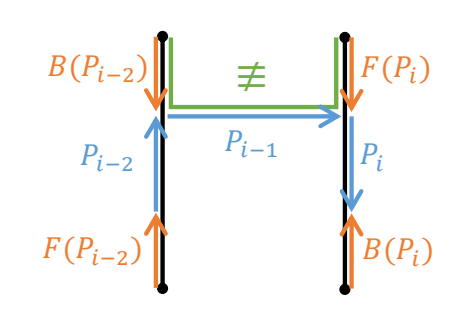

$P_{i}$ is contained in an integral path

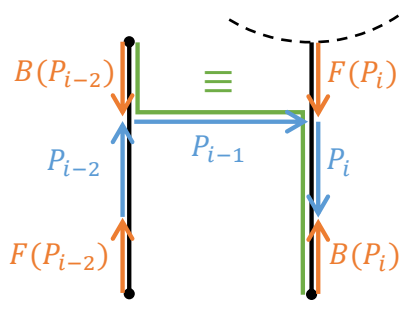

$P_{i}$ is contained in a spoke

Figure 4: Conditions for alternating paths.

1. $t(P) \in A \backslash V(y)$, and $T(P)$ is conflicting.

2. $t(P)$ is contained in a half-integral cycle, but in no spokes (i.e., $\left.t(P) \in V(y) \backslash V_{1}(y)\right)$.

3. $t(P)$ is contained in a spoke $S$, and the following two conditions are satisfied.

(a) $T(P) \not \equiv B(t(P))$.

(b) For any $P_{j}$ contained in the spoke $S, t(P)$ is contained in $F\left(P_{j}\right)$.

Definition 4. Let $P=P_{1} \circ \cdots \circ P_{p}$ and $Q=Q_{1} \circ \cdots \circ Q_{q}$ be a pair of $y$-alternating paths ending at the same vertex. $(P, Q)$ is called a $y$-augmenting pair if it satisfies all the following conditions.

1. $P$ and $Q$ can be written as $P=R \circ P^{\prime}$ and $Q=R \circ Q^{\prime}$, respectively, for some walk $R$ such that $P^{\prime}$ and $Q^{\prime}$ share no edges.

2. $T(P) \not \equiv T(Q)$.

3. At least one of $p$ and $q$ is odd, and if both of $p$ and $q$ are odd, $t(P) \notin V(y)$.

4. For any $P_{i}$ contained in an integral path, none of the $Q_{j}$ 's are contained in $B\left(P_{i}\right)$ in the opposite direction. Moreover, none of the $Q_{j}$ 's are contained in $F\left(P_{i}\right)$ in the same direction if $B\left(P_{i-2}\right) \circ P_{i-1} \not \equiv B\left(P_{i}\right) \circ P_{i}^{-1}$. The symmetric condition holds for any $Q_{i}$ contained in an integral path.

Note that for any $y$-alternating path $P$, the condition 4 is always satisfied against $(P, P)$. Therefore, in testing the condition 4 against $(P, Q)$, we only need to test pairs $\left(P_{i}, Q_{j}\right)$ such that at least one of $P_{i}$ or $Q_{j}$ is not contained in the common prefix $R$.

Using a $y$-augmenting path/pair, we can improve a basic $\mathcal{F}$-packing $y$ in linear time by the following lemmas, whose proofs are given in Section 3.4.

Lemma 2. Given a basic $\mathcal{F}$-packing y and a y-augmenting path, a basic $\mathcal{F}$-packing of size at least $|y|+\frac{1}{2}$ can be constructed in linear time.

Lemma 3. Given a basic $\mathcal{F}$-packing y and a y-augmenting pair, a basic $\mathcal{F}$-packing of size $|y|+\frac{1}{2}$ can be constructed in linear time.

Figure 5 illustrates an example of augmentations. We use the following example which corresponds to the internally-disjoint $A^{\prime}$-paths with $A^{\prime}=\{a, b, c, d\}$. Every vertex $u$ has the same domain $D(u)=\{a, b, c, d\}$ and each edge $u v$ has an equality (identity permutation) constraint 

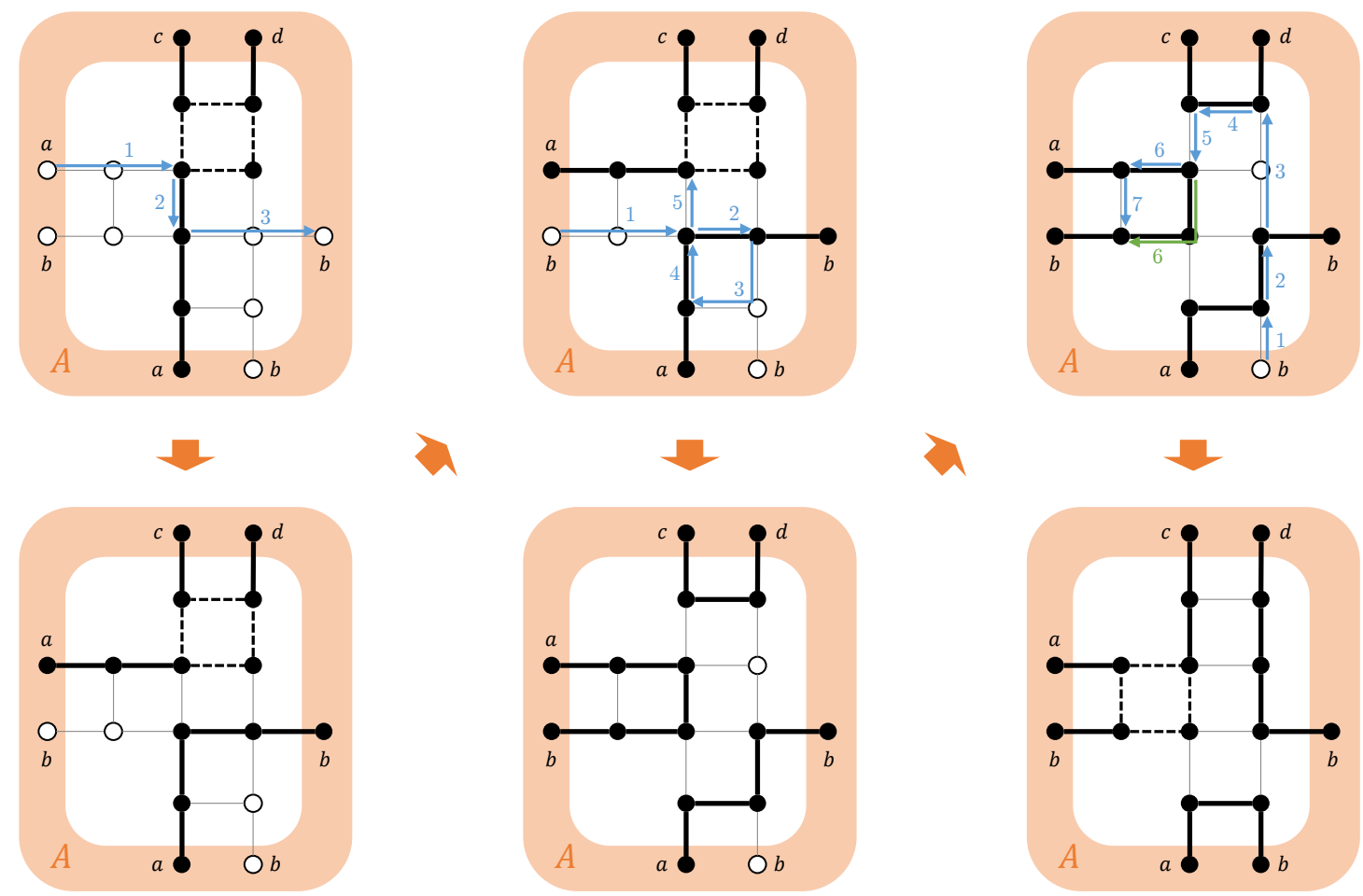

Figure 5: Example of augmentations. Thick solid lines denote edges of weight 1, dashed lines denote edges of weight $\frac{1}{2}$, and thin solid lines denote edges of weight 0 . Blue or green arrows denote segments of augmenting paths/pairs. A number $i$ beside an arrow means that it is the $i$-th segment.

$\varphi(u)=\varphi(v)$. An alphabet beside a vertex $u \in A$ shows the value $\varphi_{A}(u)$. Any walks starting from $A$ is implicational, and a walk $W$ is conflicting if and only if $\varphi_{A}(s(W)) \neq \varphi_{A}(t(W))$. Roughly speaking, for an augmenting path $P$ connecting two distinct vertices in $A$ (i.e., when it satisfies the condition 1 of Definition 3), we take the symmetric difference of $E(y)$ and $E(P)$ (Figure 5, left). For an augmenting path $P$ ending at a vertex on a wheel (i.e., when it satisfies the condition 2 or 3 of Definition 3), we take the symmetric difference and then decompose the wheel into integral paths (Figure 5, middle). For an augmenting pair $\left(R \circ P^{\prime}, R \circ Q^{\prime}\right)$ with a common prefix $R$, we take the symmetric difference of $E(y)$ and $E(R)$, and then introduce a new wheel whose half-integral cycle is $P^{\prime} \circ Q^{\prime-1}$ (Figure 5, right). In addition to this basic augmentation strategy, we need several operations in ensuring that the obtained packing is a basic $\mathcal{F}$-packing. See the proofs in Section 3.4 for more detail.

\subsection{Finding Augmenting Path/Pair}

In this subsection, we propose an algorithm for computing a $y$-augmenting path or pair. Algorithm 1 describes a rough sketch of the algorithm. We will describe later the details of an efficient implementation using the incremental-test oracle. Note that in this subsection, we prove only the soundness of the algorithm (i.e., the algorithm never returns a path or pair which is not $y$ augmenting). The completeness of the algorithm (i.e., the algorithm always finds a $y$-augmenting path or pair if exists) follows from Lemma 7 proved in the next subsection. 


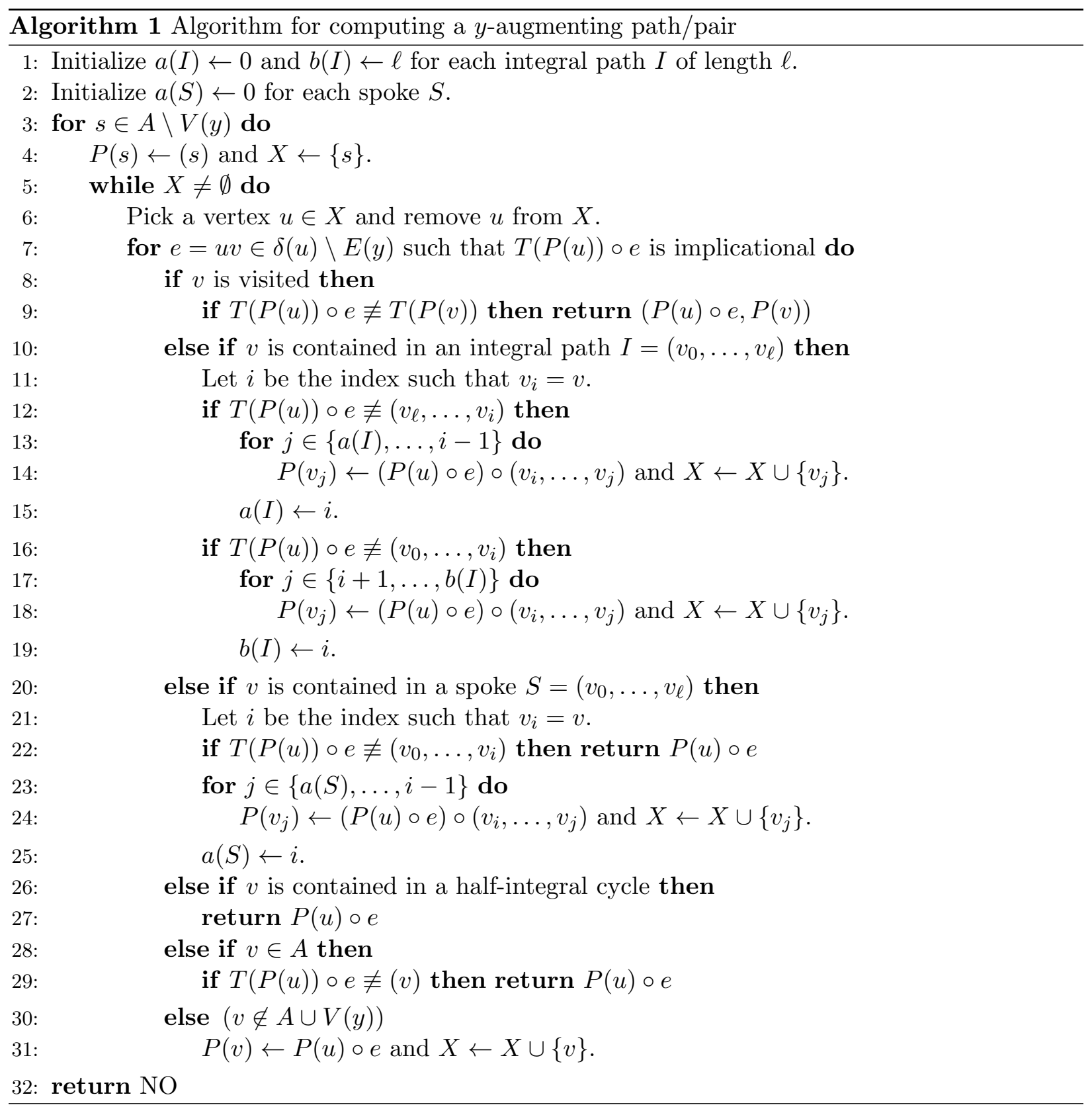

Lemma 4. Any path returned by Algorithm 1 is a y-augmenting path, and any pair returned by the algorithm is a y-augmenting pair.

In the algorithm, we hold two indices $a(I)$ and $b(I)$ for each integral path $I$ initialized as $a(I)=0$ and $b(I)=\ell$, respectively, where $\ell$ is the length of $I$; an index $a(S)$ for each spoke $S$ initialized as $a(S)=0$; a set of active vertices $X$ initialized as $X=\emptyset$; and a $y$-alternating path $P(v)$ for each visited vertex $v$, where we call a vertex $v$ visited if $v$ has been ever pushed into $X$. We define boundaries as the set of vertices consisting of $v_{a(I)}$ and $v_{b(I)}$ for each integral path $I=\left(v_{0}, \ldots, v_{\ell}\right)$ and $v_{a(S)}$ for each spoke $S=\left(v_{0}, \ldots, v_{\ell}\right)$. We preserve the following invariants during the execution 
of the algorithm.

Lemma 5. The following invariants hold at any iteration of Algorithm 1.

1. For any visited $v$, the following holds:

(a) $P(v)$ is a y-alternating path,

(b) $V(P(v))$ contains only visited vertices or boundaries, and

(c) $P(v)$ has an odd number of segments if and only if $v \notin A \cup V(y)$.

2. For any visited $u$ and $v$ with $s(P(u))=s(P(v)),(P(u), P(v))$ satisfies the condition 1 of $y$-augmenting pairs (Definition 4).

3. For any integral path $I=\left(v_{0}, \ldots, v_{\ell}\right)$, the following holds:

(a) $a(I) \leq b(I)$,

(b) $v_{i}$ is visited if and only if $i<a(I)$ or $b(I)<i$, and

(c) for any $P(v)$ with segments $P_{1} \circ \cdots \circ P_{p}$ and any segment $P_{i}$ contained in $I, P_{i}$ is contained in $\left(v_{a(I)}, \ldots, v_{0}\right)$ or $\left(v_{b(I)}, \ldots, v_{\ell}\right)$ in these directions; moreover, if $B\left(P_{i-2}\right) \circ P_{i-1} \not \equiv$ $B\left(P_{i}\right) \circ P_{i}^{-1}$ holds, then $s\left(P_{i}\right)=v_{a(I)}=v_{b(I)}$ holds.

4. For any spoke $S=\left(v_{0}, \ldots, v_{\ell}\right)$, the following holds:

(a) $v_{i}$ is visited if and only if $i<a(S)$, and

(b) for any $P(v)$, all the segments of $P(v)$ contained in $S$ are contained in $\left(v_{a(S)}, \ldots, v_{0}\right)$.

We now prove Lemma 4 and 5. All the invariants are clearly satisfied at the beginning. In each iteration, we pick an arbitrary vertex $u$ from $X$ (line 6). From the invariant $1, P(u)$ is a $y$-alternating path. We then iterate over the edges $e=u v \in \delta(u) \backslash E(y)$ such that $T(P(u)) \circ e$ is implicational.

When $v$ is already visited and $T(P(u)) \circ e \not \equiv T(P(v))$ holds, we return a pair $(P(u) \circ e, P(v))$ (line 9).

Claim 1. $(P(v), P(u) \circ e)$ returned at line 9 is a y-augmenting pair.

Proof. First, we prove $e \notin E(P(u))$, which implies that $P(u) \circ e$ is a $y$-alternating path. If $e \in$ $E(P(u)), e^{-1}$ is either the last edge of $P(u)$ or the last edge of an odd segment $P_{i}$ of $P(u):=P_{1} \circ \cdots \circ$ $P_{p}$. In the former case, $T(P(u)) \circ e=T(P(v)) \circ e^{-1} \circ e \equiv T(P(v))$ holds, which is a contradiction. In the latter case, from the invariant 2 against $(P(u), P(v))$, we have $P(v)=P_{1} \circ \cdots \circ P_{i-1} \circ W$, where $W$ is the path satisfying $W \circ e^{-1}=P_{i}$. Because $P_{i+1}$ and $P_{p}$ are contained in the same integral path or spoke in the same direction, we have $B\left(P_{i-1}\right) \circ P_{i} \equiv B\left(P_{i+1}\right) \circ P_{i+1}^{-1}$ from the condition 5 of $y$-alternating paths. Therefore, we have $T(P(u)) \circ e=B\left(P_{p}\right) \circ e=B\left(P_{i+1}\right) \circ P_{i+1}^{-1} \circ e \equiv$ $B\left(P_{i-1}\right) \circ P_{i} \circ e=B\left(P_{i-1}\right) \circ W \circ e^{-1} \circ e=T(P(v)) \circ e^{-1} \circ e \equiv T(P(v))$, which is a contradiction.

Next, we prove that $(P(v), P(u) \circ e)$ satisfies all the conditions of the $y$-augmenting pairs (Definition 4). Suppose that $s(P(u)) \neq s(P(v))$ holds. Then, the vertex $v$ has already been popped from $X$ and, thus, we have $P(u)=P(v) \circ e^{-1}$, which is a contradiction. Therefore, from the invariant 2 , the condition 1 is satisfied. The condition 2 is satisfied because $T(P(v)) \not \equiv T(P(u)) \circ e=T(P(u) \circ e)$. Meanwhile, the condition 3 is satisfied because the number of segments of $P(u) \circ e$ is odd and because, from the invariant $1, v \in V(y)$ implies that the number of segments of $P(v)$ is even. The condition 4 follows from the invariant 3 . 
When $v$ is not visited, we consider five cases: (Case 1) $v$ is contained in an integral path; (Case 2) $v$ is contained in a spoke; (Case 3) $v$ is contained in a half-integral cycle (but not in spokes); (Case 4) $v \in A$ (but not in $V(y)$ ); or (Case 5) $v \notin A \cup V(y)$. Note that $e$ is not contained in $E(P(u))$ when $v$ is not visited. Therefore, $P(u) \circ e$ is a $y$-alternating path. In case 3 , we return $P(u) \circ e$, which is a $y$-augmenting path satisfying the condition 2 of Definition 3 . In case 4 , if $T(P(u)) \circ e \not \equiv(v)$ holds, we return $P(u) \circ e$, which is a $y$-augmenting path satisfying the condition 1 of Definition 3, and otherwise, we do nothing. In case 5, we set $P(v) \leftarrow P(u) \circ e$ and insert $v$ into $X$ (line 31). Because $e \notin E(y)$ holds, all the invariants are clearly preserved. Finally, we consider the remaining two cases.

(Case 1) Let $I=\left(v_{0}, \ldots, v_{\ell}\right)$ be the integral path containing $v$ and let $i$ be the index such that $v_{i}=v$. Because $v$ is not visited, $a(I) \leq i \leq b(I)$ holds from the invariant 3. If $T(P(u)) \circ e \not \equiv$ $\left(v_{\ell}, \ldots, v_{i}\right)$ holds, we set $P\left(v_{j}\right) \leftarrow(P(u) \circ e) \circ\left(v_{i}, \ldots, v_{j}\right)$ and insert $v_{j}$ into $X$ for each index $j \in\{a(I), \ldots, i-1\}$, and then update $a(I) \leftarrow i$ (lines 12-15). Similarly, if $T(P(u)) \circ e \not \equiv\left(v_{0}, \ldots, v_{i}\right)$ holds, we set $P\left(v_{j}\right) \leftarrow(P(u) \circ e) \circ\left(v_{i}, \ldots, v_{j}\right)$ and insert $v_{j}$ into $X$ for each index $j \in\{i+1, \ldots, b(I)\}$, and then update $b(I) \leftarrow i$ (lines 16-19).

Claim 2. The lines 12-19 preserve all the invariants.

Proof. The invariants 1b, 1c, 2, 3a, 3b, and 4 are clearly preserved. Let $a(I)$ and $b(I)$ denote the indices before the updates. For an index $j \in\{a(I), \ldots, i-1\}$, let $P_{1} \circ \cdots \circ P_{p}$ be the segments of $P\left(v_{j}\right)$. If $B\left(P_{p-2}\right) \circ P_{p-1} \not \equiv B\left(P_{p}\right) \circ P_{p}^{-1}$ holds, we have $T(P(u)) \circ e=B\left(P_{p-2}\right) \circ P_{p-1} \not \equiv$ $B\left(P_{p}\right) \circ P_{p}^{-1}=\left(v_{0}, \ldots, v_{i}\right)$. Therefore, both $a(I)$ and $b(I)$ are updated to $i$. The same argument applies to the case of $j \in\{i+1, \ldots, b(I)\}$. Thus, the invariant $3 \mathrm{c}$ is preserved.

Finally, we prove the invariant 1 a by showing that $P\left(v_{j}\right)$ is a $y$-alternating path for any newly visited $v_{j}$. The conditions 1-4 of $y$-alternating paths (Definition 2) are clearly satisfied. Consider the case of $j \in\{a(I), \ldots, i-1\}$. The proof for the case of $j \in\{i+1, \ldots, b(I)\}$ is symmetric. Let $P_{1} \circ \cdots \circ P_{p}$ be the segments of $P\left(v_{j}\right)$. Because $B\left(P_{p-2}\right) \circ P_{p-1}=T(P(u)) \circ e \not \equiv\left(v_{\ell}, \ldots, v_{i}\right)=F\left(P_{p}\right)$ holds, the condition 5 is satisfied for $p$. Let $P_{k}$ be a segment with $k<p$ contained in the same integral path $I$. From the invariant $3 c$ and because $a(I) \leq j<i \leq b(I), P_{p}=\left(v_{i}, \ldots, v_{j}\right)$ is contained in $F\left(P_{k}\right)$, and $B\left(P_{k-2}\right) \circ P_{k-1} \equiv B\left(P_{k}\right) \circ P_{k}^{-1}$ holds. Therefore, the condition 5 is satisfied for $k$.

(Case 2) Let $S=\left(v_{0}, \ldots, v_{\ell}\right)$ be the spoke containing $v$ and let $i$ be the index such that $v_{i}=v$. Because $v$ is not visited, $a(S) \leq i$ holds from the invariant 4 . If $T(P(u)) \circ e \not \equiv\left(v_{0}, \ldots, v_{i}\right)$ holds, we return a path $P(u) \circ e$ (line 22); otherwise, we set $P\left(v_{j}\right) \leftarrow(P(u) \circ e) \circ\left(v_{i}, \ldots, v_{j}\right)$ and insert $v_{j}$ into $X$ for each index $j \in\{a(S), \ldots, i-1\}$, and then update $a(S) \leftarrow i$ (lines 23-25).

Claim 3. $P(u) \circ$ e returned at line 22 is a y-augmenting path.

Proof. Because $T(P(u)) \circ e \not \equiv\left(v_{0}, \ldots, v_{i}\right)=B(t(P(u) \circ e))$ holds, the condition 3a of the $y$-augment paths (Definition 3) is satisfied. From the invariant 4, for any segment $P_{k}$ of $P(u) \circ e$ contained in the spoke $S, t(P(u) \circ e)=v_{i}$ is contained in $F\left(P_{k}\right)$. Therefore, the condition 3b is satisfied.

Claim 4. Lines 23-25 preserve all the invariants.

Proof. All the invariants, except for the 1a, are clearly preserved. We prove the invariant 1a by showing that $P\left(v_{j}\right)$ is a $y$-alternating path for any newly visited $v_{j}$. The conditions $1-4$ of the 
$y$-alternating paths (Definition 2) are clearly satisfied. Let $P_{1} \circ \cdots \circ P_{p}$ be the segments of $P\left(v_{j}\right)$. Because $B\left(P_{p-2}\right) \circ P_{p-1}=T(P(u) \circ e) \equiv\left(v_{0}, \ldots, v_{i}\right)=B\left(P_{p}\right) \circ P_{p}^{-1}$ holds, the condition 5 is satisfied for $p$. Let $P_{k}$ be a segment with $k<p$ contained in the same spoke $S$. From the invariant $4, P_{k}$ must be contained in $\left(v_{a(S)}, \ldots, v_{0}\right)$. Therefore, $P_{p}$ is contained in $F\left(P_{k}\right)$. Thus, the condition 5 is satisfied for $k$.

\section{Implementation Detail}

We exploit the incremental-test oracle as follows to achieve the linear-time complexity. For each integral path $I=\left(v_{0}, \ldots, v_{\ell}\right)$ and for each index $i \in\{0, \ldots, \ell\}$, we precompute $\mathcal{A}^{*}\left(\left(v_{0}, \ldots, v_{i}\right)\right)$ and $\mathcal{A}^{*}\left(\left(v_{\ell}, \ldots, v_{i}\right)\right)$. For each spoke $S=\left(v_{0}, \ldots, v_{\ell}\right)$ and for each index $i \in\{0, \ldots, \ell\}$, we precompute $\mathcal{A}^{*}\left(\left(v_{0}, \ldots, v_{i}\right)\right)$. These precomputations can be done in $O(n T)$ time, where $T$ is the running time of the oracle.

For each visited vertex $v$, instead of explicitly holding $P(v)$, we hold (1) $\operatorname{prev}(v)$ that represents the edge picked at line 7 in the iteration when $P(v)$ is assigned and $(2)$ tail $(v):=\mathcal{A}^{*}(T(P(v)))$. When the algorithm finds a $y$-augmenting path or pair, we restore $P(v)$ using the table of $\operatorname{prev}(v)$ in $O(n)$ time. For an edge $e=u v \in \delta(u) \backslash E(y)$, we have $\mathcal{A}^{*}(T(P(u)) \circ e)=\mathcal{A}(\operatorname{tail}(u)$, e), which can be computed in $O(T)$ time. For any index $j$ picked at line 13 or 23 , we have tail $\left(v_{j}\right)=$ $\mathcal{A}^{*}\left(T\left(P\left(v_{j}\right)\right)\right)=\mathcal{A}^{*}\left(\left(v_{0}, \ldots, v_{j}\right)\right)$, which has been precomputed. For any index $j$ picked at line 17 , we have tail $\left(v_{j}\right)=\mathcal{A}^{*}\left(T\left(P\left(v_{j}\right)\right)\right)=\mathcal{A}^{*}\left(\left(v_{\ell}, \ldots, v_{j}\right)\right)$, which has been precomputed. When $v \notin$ $A \cup V(y)$, we have $\operatorname{tail}(v)=\mathcal{A}(\operatorname{tail}(u), e)$. Thus, we can compute tail $(w)$ in $O(T)$ time for each newly visited vertex $w$.

Next, we show that each of the equivalence tests in the algorithm can be done in $O(T)$ time. Let $e=u v$ be the edge picked at line 7. First, we consider the equivalence test at line 9 . If $\operatorname{prev}(v)=e^{-1}$ holds, we have $T(P(u)) \circ e \equiv T(P(v))$; otherwise, $(T(P(u)) \circ e, T(P(v)))$ forms a single-branching pair from the invariant 2, for which we can test $T(P(u)) \circ e \not \equiv T(P(v))$ by asking $\mathcal{T}(\mathcal{A}(\operatorname{tail}(u), e), \operatorname{tail}(v))$ in $O(T)$ time. Next, we consider the equivalence test at line 12 . Because $\left(T(P(u) \circ e),\left(v_{\ell}, \ldots, v_{i}\right)\right)$ forms a single-branching pair, we can test $T(P(u)) \circ e \not \equiv\left(v_{\ell}, \ldots, v_{i}\right)$ by asking $\mathcal{T}\left(\mathcal{A}(\operatorname{tail}(u), e), \mathcal{A}^{*}\left(\left(v_{\ell}, \ldots, v_{i}\right)\right)\right)$. This can be done in $O(T)$ time because $\mathcal{A}^{*}\left(\left(v_{\ell}, \ldots, v_{i}\right)\right)$ has been precomputed. The same argument applies to the equivalence tests at lines 16 and 22 . The equivalence test at line 29 can be done in $O(T)$ time by asking $\mathcal{T}(\mathcal{A}(\operatorname{tail}(u), e), \mathcal{I}(v))$.

Now, we have shown that $\operatorname{tail}(v)$ can be computed in $O(T)$ time for each visited vertex $v$, and that all the equivalence tests can be done in $O(T)$ time for each edge picked at line 7 . Because each vertex is pushed into $X$ at most once and because each edge is processed at most twice (in both directions), we obtain the following lemma.

Lemma 6. Given an $O(T)$-time incremental-test oracle, Algorithm 1 runs in $O(m T)$ time.

\subsection{Constructing Half-Integral $\mathcal{F}$-Cover}

In this subsection, we prove that if Algorithm 1 fails to find a $y$-augmenting path or pair, we can construct a half-integral $\mathcal{F}$-cover of the same size as follows. Let $a$ and $b$ be the tables used in Algorithm 1. First, we initialize $x(v) \leftarrow 0$ for all $v \in V$. For each integral path $I=\left(v_{0}, \ldots, v_{\ell}\right)$, we set $x\left(v_{a(I)}\right) \leftarrow \frac{1}{2}$ and $x\left(v_{b(I)}\right) \leftarrow \frac{1}{2}$ if $a(I) \neq b(I)$, and set $x\left(v_{a(I)}\right) \leftarrow 1$ if $a(I)=b(I)$. We set $x\left(v_{a(S)}\right) \leftarrow \frac{1}{2}$ for each spoke $S=\left(v_{0}, \ldots, v_{\ell}\right)$. From the construction, we have $|x|=|y|$. We show that the function $x$ is an $\mathcal{F}$-cover. 
Lemma 7. If Algorithm 1 fails to find a y-augmenting path or pair, the function $x$ constructed as above is a half-integral $\mathcal{F}$-cover of size $|y|$.

We use the following lemma to prove this lemma.

Lemma 8. For any implicational walk $Q$ with $x(V(Q))=0$, the vertex $t(Q)$ is visited and $T(P(t(Q))) \equiv Q$ holds.

Proof. We prove the lemma by induction on the length of $Q$. For any vertex $s \in A$ with $x(s)=0$, $s$ is visited, and $T(P(s))=(s)$ holds. Therefore, the statement holds when the length of $Q$ is zero. Let $Q=Q^{\prime} \circ u v$ be an implicational walk with $x(V(Q))=0$. From the induction hypothesis, $u$ is visited, and $T(P(u)) \equiv Q^{\prime}$ holds. Therefore, we have $T(P(u)) \circ u v \equiv Q^{\prime} \circ u v=Q$. If $u v \notin E(y)$ and $v$ is contained in a half-integral cycle but in no spokes, the algorithm returns a $y$-augmenting path at line 27. We consider the following four cases: (Case 1) $v \notin V(y)$; (Case 2) $u v \notin E(y)$ and $v$ is contained in an integral path; (Case 3) $u v \notin E(y)$ and $v$ is contained in a spoke; or (Case 4) $u v \in E(y)$.

(Case 1) Consider the iteration when $u$ is picked at line 6. If $v$ is already visited, $T(P(u)) \circ u v \equiv$ $T(P(v))$ holds because, otherwise, the algorithm returns a $y$-augmenting pair. Therefore, we have $T(P(v)) \equiv T(P(u)) \circ u v \equiv Q$. If $v$ is not visited yet and $v \in A$, we have $T(P(u)) \circ u v \equiv(v)$. Therefore, we have $T(P(v))=(v) \equiv T(P(u)) \circ u v \equiv Q$. If $v$ is not visited yet and $v \notin A$, then $v$ becomes visited, and we have $T(P(v))=T(P(u)) \circ u v \equiv Q$.

(Case 2) Let $I=\left(v_{0}, \ldots, v_{\ell}\right)$ be the integral path containing $v$ and let $i$ be the index such that $v_{i}=v$. Consider the iteration when $u$ is picked at line 6 . Let $a(I)$ and $b(I)$ denote the values at the beginning of this iteration (hence, $x\left(v_{a(I)}\right)$ and $x\left(v_{b(I)}\right)$ might be zero). If $i<a(I)$ holds, then $v$ is already visited, and $T(P(v))=\left(v_{0}, \ldots, v_{i}\right)$ holds. If $T(P(u)) \circ u v \not \equiv\left(v_{0}, \ldots, v_{i}\right)$ additionally holds, the algorithm returns a $y$-augmenting pair at line 9 . Therefore, we have $T(P(v))=\left(v_{0}, \ldots, v_{i}\right) \equiv$ $T(P(u)) \circ u v \equiv Q$. The same argument applies to the case of $i>b(I)$.

We now consider the remaining case that $a(I) \leq i \leq b(I)$ holds. Because $\left(v_{0}, \ldots, v_{i}\right) \not \equiv$ $\left(v_{\ell}, \ldots, v_{i}\right)$ holds, at least one of $T(P(u)) \circ u v \not \equiv\left(v_{0}, \ldots, v_{i}\right)$ or $T(P(u)) \circ u v \not \equiv\left(v_{\ell}, \ldots, v_{i}\right)$ holds. If both hold, $a(I)$ and $b(I)$ are both set to $i$ after this iteration. Therefore, we have $x(v)=1$, which is a contradiction. If $T(P(u)) \circ u v \equiv\left(v_{0}, \ldots, v_{i}\right)$ holds, $a(I)$ is set to $i$ after this iteration. Because $x(v)=0, a(I)$ must be greater than $i$ at the end of the algorithm. Therefore, we have $T(P(v))=\left(v_{0}, \ldots, v_{i}\right) \equiv T(P(u)) \circ u v \equiv Q$. The same argument applies to the case of $T(P(u)) \circ u v \equiv\left(v_{\ell}, \ldots, v_{i}\right)$.

(Case 3) Let $S=\left(v_{0}, \ldots, v_{\ell}\right)$ be the spoke containing $v$ and $i$ be the index such that $v_{i}=v$. Consider the iteration when $u$ is picked at line 6. Let $a(S)$ denote the value at the beginning of this iteration. If $i<a(S)$ holds, then $v$ is already visited, and $T(P(v))=\left(v_{0}, \ldots, v_{i}\right)$ holds. If $T(P(u)) \circ u v \not \equiv\left(v_{0}, \ldots, v_{i}\right)$ additionally holds, the algorithm returns a $y$-augmenting pair at line 9 . Therefore, we have $T(P(v))=\left(v_{0}, \ldots, v_{i}\right) \equiv T(P(u)) \circ u v \equiv Q$.

We now consider the remaining case that $a(I) \leq i$ holds. If $T(P(u)) \circ u v \not \equiv\left(v_{0}, \ldots, v_{i}\right)$ holds, the algorithm returns a $y$-augmenting path at line 22. Therefore, $T(P(u)) \circ u v \equiv\left(v_{0}, \ldots, v_{i}\right)$ holds and $a(I)$ is set to $i$ after this iteration. Because $x(v)=0, a(I)$ must be greater than $i$ at the end of the algorithm. Therefore, we have $T(P(v))=\left(v_{0}, \ldots, v_{i}\right) \equiv T(P(u)) \circ u v \equiv Q$. 
(Case 4) Note that in this case, $u v$ must be contained in an integral path or a spoke because the vertices contained in half-integral cycles are never visited. Let $\left(v_{0}, \ldots, v_{\ell}\right)$ be the integral path or the spoke containing $u v$ and let $i$ and $j$ be the indices such that $v_{i}=v$ and $v_{j}=u$. Because $u$ is visited, $T(P(u))$ is either $\left(v_{0}, \ldots, v_{j}\right)$ or $\left(v_{\ell}, \ldots, v_{j}\right)$, and w.l.o.g., we can assume the former case. If $i=j-1$, then $v$ is also visited, and we have $T(P(v))=\left(v_{0}, \ldots, v_{j-1}\right) \equiv\left(v_{0}, \ldots, v_{j}\right) \circ u v=T(P(u)) \circ u v \equiv Q$. If $i=j+1$ and $v$ is not visited, then we have $x(v) \geq \frac{1}{2}$, which is a contradiction. Therefore, $v$ is also visited, and we have $T(P(v))=\left(v_{0}, \ldots, v_{j+1}\right)=\left(v_{0}, \ldots, v_{j}\right) \circ u v=T(P(u)) \circ u v \equiv Q$.

Proof of Lemma \%. Suppose that there exists a conflicting walk $W \in \mathcal{F}$ with $x(V(W))<1$. Because $x(v) \in\left\{0, \frac{1}{2}, 1\right\}$ holds, $x(V(W))$ is either 0 or $\frac{1}{2}$. If $x(V(W))=0$, by applying Lemma 8 against $W$, we have $W \equiv T(P(t(W)))=(t(W))$, which is a contradiction. If $x(V(W))=\frac{1}{2}$, let $v$ be the vertex with $x(v)=\frac{1}{2}$ on $W$. By splitting $W$ at $v$, we obtain two implicational walks $Q_{1}$ and $Q_{2}$ such that $W=Q_{1} \circ Q_{2}^{-1}$ and $t\left(Q_{1}\right)=t\left(Q_{2}\right)=v$. Because $x(v)=\frac{1}{2}, v$ is contained in an integral path or a spoke $I=\left(v_{0}, \ldots, v_{\ell}\right)$. Let $i$ be the index such that $v_{i}=v$ and, w.l.o.g., we can assume that $a(I)=i$. If $I$ is an integral path, $b(I)$ must be greater than $i$ because, otherwise, we have $x(v)=1$.

If the length of $Q_{1}$ is zero, $i$ is either 0 or $\ell$. In the latter case, $I$ is an integral path, and $\ell=i=a(I)<b(I) \leq \ell$, which is a contradiction. Thus, we have $Q_{1}=\left(v_{0}\right)$.

If $Q_{1}$ has a positive length, let $Q_{1}=R \circ u v$. By applying Lemma 8 against $R, u$ is visited, and $Q_{1}=R \circ u v \equiv T(P(u)) \circ u v$ holds. If $u v$ is contained in $I, u$ must be $v_{i-1}$ because $v_{i+1}$ is not visited. Therefore, we have $Q_{1} \equiv T(P(u)) \circ u v=\left(v_{0}, \ldots, v_{i-1}\right) \circ u v=\left(v_{0}, \ldots, v_{i}\right)$. If $u v$ is not contained in $I$, consider the iteration when $u$ is picked at line 6 . We then have $Q_{1} \equiv T(P(u)) \circ u v \equiv\left(v_{0}, \ldots, v_{i}\right)$ because, otherwise, $b(I)$ is set to $i$, or the algorithm returns a $y$-augmenting path at line 22 .

We now have proved that $Q_{1} \equiv\left(v_{0}, \ldots, v_{i}\right)$ holds. By applying the same argument to $Q_{2}$, we have $Q_{2} \equiv\left(v_{0}, \ldots, v_{i}\right)$. Therefore, we have $Q_{1} \equiv Q_{2}$, which is a contradiction.

\subsection{Augmentation}

\subsubsection{Simplification of Alternating Path}

Before the proofs of Lemmas 2 and 3, we introduce a useful procedure to simplify an augmenting path/pair obtained by Algorithm 1. We first define such an operation in a formal manner and prove the validity just after the definition.

Definition 5. For a basic $\mathcal{F}$-packing $y$ and a $y$-alternating path $P=P_{1} \circ \cdots \circ P_{p}$ with $p \geq 2$, the simplification $\left(y^{\prime}, P^{\prime}\right)$ of $(y, P)$ is defined as follows (Figure 6).

- A function $y^{\prime}$ is constructed from $y$ as follows:

- if $P_{2}$ is contained in an integral path $I$, replace $I$ with an integral path $P_{1} \circ F\left(P_{2}\right)^{-1}$;

- if $P_{2}$ is contained in a spoke $S$, replace $S$ with a spoke $P_{1} \circ F\left(P_{2}\right)^{-1}$.

- A walk $P^{\prime}$ is defined as a concatenation $\left(B_{y}\left(P_{2}\right) \circ P_{3}\right) \circ P_{4} \circ P_{5} \circ \cdots \circ P_{p}$ of $\max \{p-2,1\}$ paths $^{14}$.

Lemma 9. For any basic $\mathcal{F}$-packing $y$ and any $y$-alternating path $P=P_{1} \circ \cdots \circ P_{p}$ with $p \geq 2$, the simplification $\left(y^{\prime}, P^{\prime}\right)$ of $(y, P)$ satisfies the following.

\footnotetext{
${ }^{14}$ When $p=2, P^{\prime}=B_{y}\left(P_{2}\right)$.
} 

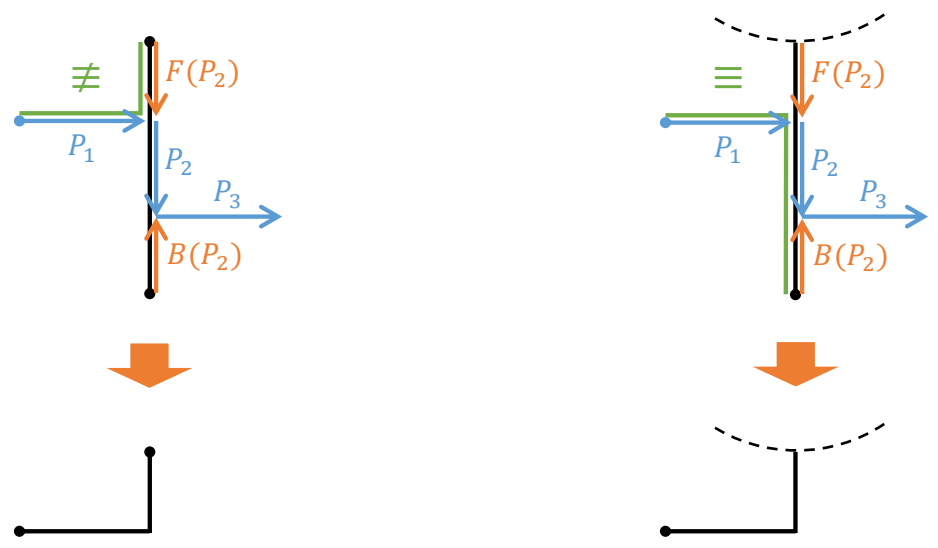

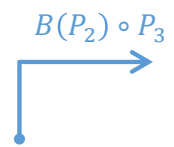

$P_{2}$ is contained in an integral path
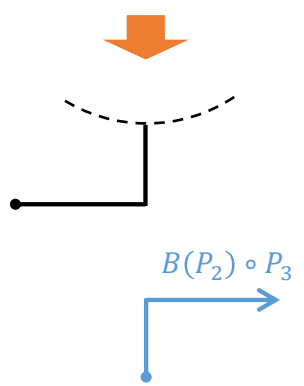

$P_{2}$ is contained in a spoke

Figure 6: Simplification of a basic $\mathcal{F}$-packing and an alternating path.

1. $y^{\prime}$ is a basic $\mathcal{F}$-packing with $\left|y^{\prime}\right|=|y|$.

2. For any even $i \geq 4$, the following holds.

(a) If $P_{i}$ is contained in an integral path in $y$, it is also contained in an integral path in $y^{\prime}$. Moreover, $F_{y^{\prime}}\left(P_{i}\right) \equiv F_{y}\left(P_{i}\right)$ and $B_{y^{\prime}}\left(P_{i}\right) \equiv B_{y}\left(P_{i}\right)$ hold.

(b) If $P_{i}$ is contained in a spoke in $y$, it is also contained in a spoke in $y^{\prime}$ in the direction toward $A$. Moreover, $F_{y^{\prime}}\left(P_{i}\right)=F_{y}\left(P_{i}\right)$ and $B_{y^{\prime}}\left(P_{i}\right) \equiv B_{y}\left(P_{i}\right)$ hold.

3. $P^{\prime}=\left(B_{y}\left(P_{2}\right) \circ P_{3}\right) \circ P_{4} \circ P_{5} \circ \cdots \circ P_{p}$ is a $y^{\prime}$-alternating path, where $B_{y}\left(P_{2}\right) \circ P_{3}$ is the first segment, and $P_{i}(i \geq 4)$ is the $(i-2)$-th segment ${ }^{15}$.

Proof. First, we prove the first claim. When $P_{2}$ is contained in an integral path $I$, from the condition $5 \mathrm{~b}$ of the $y$-alternating paths (Definition 2), $P_{1} \not \equiv F\left(P_{2}\right)$ holds (i.e., $I^{\prime}:=P_{1} \circ F\left(P_{2}\right)^{-1} \in$ $\mathcal{F})$. Because $P_{1}$ is internally disjoint from $F\left(P_{2}\right)$ and $s\left(P_{1}\right) \notin V(y), I^{\prime}$ is a simple path. Thus, we can replace $I$ with the integral path $I^{\prime}$. When $P_{2}$ is contained in a spoke $S_{i}$ of a wheel, from the condition $5 \mathrm{c}, P_{1} \equiv B\left(P_{2}\right) \circ P_{2}^{-1}$ holds. From the definition of the wheel (Definition 1), $S_{i-1} \circ H_{i-1} \circ S_{i}^{-1}$ and $S_{i} \circ H_{i} \circ S_{i+1}^{-1}$ are both in $\mathcal{F}$. As $B\left(P_{2}\right) \circ P_{2}^{-1} \circ F\left(P_{2}\right)^{-1}=S_{i}$ holds, we have $S_{i}^{\prime}:=P_{1} \circ F\left(P_{2}\right)^{-1} \equiv S_{i}$. When the degree of the wheel is at least three, $S_{i-1} \circ H_{i-1} \circ S_{i}^{\prime-1}$ and $S_{i}^{\prime} \circ H_{i} \circ S_{i+1}^{-1}$ are both in $\mathcal{F}$. When the degree of the wheel is one, $S_{1}^{\prime} \circ H_{1} \circ S_{1}^{\prime-1}$ is in $\mathcal{F}$. Thus, this replacement of the spoke preserves the condition for the wheel.

Next, we prove the second claim. We can observe the following for any even $i \geq 4$.

- If both of $P_{2}$ and $P_{i}$ are contained in the same integral path in $y$, from the condition 5b in Definition 2, $P_{i}$ is contained in $F_{y}\left(P_{2}\right)$, and $P_{1} \equiv B_{y}\left(P_{2}\right) \circ P_{2}^{-1}$ holds. Thus, $P_{i}$ is contained in the integral path $P_{1} \circ F_{y}\left(P_{2}\right)^{-1}$ in $y^{\prime}$. If they have the same direction, $P_{2}$ is contained in

\footnotetext{
${ }^{15}$ When $p=2, P^{\prime}$ consists of the single segment $B_{y}\left(P_{2}\right)$.
} 
$B_{y}\left(P_{i}\right)$, and we can write $B_{y}\left(P_{i}\right)=\left(B_{y}\left(P_{2}\right) \circ P_{2}^{-1}\right) \circ W$ for some subpath $W$. Then, it holds that $F_{y^{\prime}}\left(P_{i}\right)=F_{y}\left(P_{i}\right)$ and $B_{y^{\prime}}\left(P_{i}\right)=P_{1} \circ W \equiv\left(B_{y}\left(P_{2}\right) \circ P_{2}^{-1}\right) \circ W=B_{y}\left(P_{i}\right)$. If they have the opposite direction, $P_{2}$ is contained in $F_{y}\left(P_{i}\right)$, and we can write $F_{y}\left(P_{i}\right)=\left(B_{y}\left(P_{2}\right) \circ P_{2}^{-1}\right) \circ W$ for some subpath $W$. Then, it holds that $F_{y^{\prime}}\left(P_{i}\right)=P_{1} \circ W \equiv\left(B_{y}\left(P_{2}\right) \circ P_{2}^{-1}\right) \circ W=F_{y}\left(P_{i}\right)$ and $B_{y^{\prime}}\left(P_{i}\right)=B_{y}\left(P_{i}\right)$.

- If both $P_{2}$ and $P_{i}$ are contained in the same spoke in $y$, from the condition 5c in Definition 2, $P_{i}$ is contained in $F_{y}\left(P_{2}\right)$, and $P_{1} \equiv B_{y}\left(P_{2}\right) \circ P_{2}^{-1}$ holds. Thus, $P_{i}$ is contained in the spoke $P_{1} \circ F_{y}\left(P_{2}\right)^{-1}$ in $y^{\prime}$. Because both of them are directed toward $A$, we can write $B_{y}\left(P_{i}\right)=$ $\left(B_{y}\left(P_{2}\right) \circ P_{2}^{-1}\right) \circ W$ for some subpath $W$. Then, it holds that $F_{y^{\prime}}\left(P_{i}\right)=F_{y}\left(P_{i}\right)$ and $B_{y^{\prime}}\left(P_{i}\right)=$ $P_{1} \circ W \equiv\left(B_{y}\left(P_{2}\right) \circ P_{2}^{-1}\right) \circ W=B_{y}\left(P_{i}\right)$.

- Otherwise, the integral path or the spoke containing $P_{i}$ does not change. Thus, we have $F_{y^{\prime}}\left(P_{i}\right)=F_{y}\left(P_{i}\right)$ and $B_{y^{\prime}}\left(P_{i}\right)=B_{y}\left(P_{i}\right)$.

Finally, we prove the third claim. Because $B_{y}\left(P_{2}\right)$ does not contain any $P_{i}$, the conditions 14 in Definition 2 are satisfied. From the second claim and the property that $A \circ W \equiv B \circ W$ holds for any implicational walks $A \circ W$ and $B$ satisfying $A \equiv B$, none of the three equivalence relations appeared in the condition 5 change. As we have seen in the proof of the second claim, $E\left(B_{y^{\prime}}\left(P_{i}\right)\right) \backslash E\left(B_{y}\left(P_{i}\right)\right) \subseteq E\left(P_{1}\right)$ and $E\left(F_{y^{\prime}}\left(P_{i}\right)\right) \backslash E\left(F_{y}\left(P_{i}\right)\right) \subseteq E\left(P_{1}\right)$ hold. Therefore, for any $i \geq 4$, none of the $P_{j}$ 's with $j>i$ are newly contained in $B_{y^{\prime}}\left(P_{i}\right)$ or $F_{y^{\prime}}\left(P_{i}\right)$. Thus, the condition 5 is satisfied.

We obtain the following corollaries from Lemma 9 by repeatedly applying the simplifying operation.

Corollary 3. Given a basic $\mathcal{F}$-packing $y$, a y-alternating path $P=P_{1} \circ \cdots \circ P_{p}$, and an even integer $p^{\prime} \leq p$, a basic $\mathcal{F}$-packing $y^{\prime}$ of the same size and a $y^{\prime}$-alternating path $P^{\prime}$ satisfying the following conditions can be constructed in linear time.

1. $P^{\prime}$ can be written as $P^{\prime}=\left(B^{\prime} \circ P_{p^{\prime}+1}\right) \circ P_{p^{\prime}+2} \circ \cdots \circ P_{p}$ for some implicational path $B^{\prime} \equiv B_{y}\left(P_{p^{\prime}}\right)$.

2. For any even $i \geq p^{\prime}+2$, the following holds.

(a) If $P_{i}$ is contained in an integral path in $y$, it is also contained in an integral path in $y^{\prime}$. Moreover, $F_{y^{\prime}}\left(P_{i}\right) \equiv F_{y}\left(P_{i}\right)$ and $B_{y^{\prime}}\left(P_{i}\right) \equiv B_{y}\left(P_{i}\right)$ hold.

(b) If $P_{i}$ is contained in a spoke in $y$, it is also contained in a spoke in $y^{\prime}$ in the direction toward A. Moreover, $F_{y^{\prime}}\left(P_{i}\right)=F_{y}\left(P_{i}\right)$ and $B_{y^{\prime}}\left(P_{i}\right) \equiv B_{y}\left(P_{i}\right)$ hold.

Corollary 4. Given a basic $\mathcal{F}$-packing y and a y-alternating path $P=P_{1} \circ \cdots \circ P_{p}$, a basic $\mathcal{F}$ packing $y^{\prime}$ of the same size and a single-segment $y^{\prime}$-alternating path $P^{\prime}$ satisfying $P^{\prime} \equiv T_{y}(P)$ can be constructed in linear time.

\subsubsection{Augmentation by Augmenting Path}

The subsection aims to prove Lemma 2. 

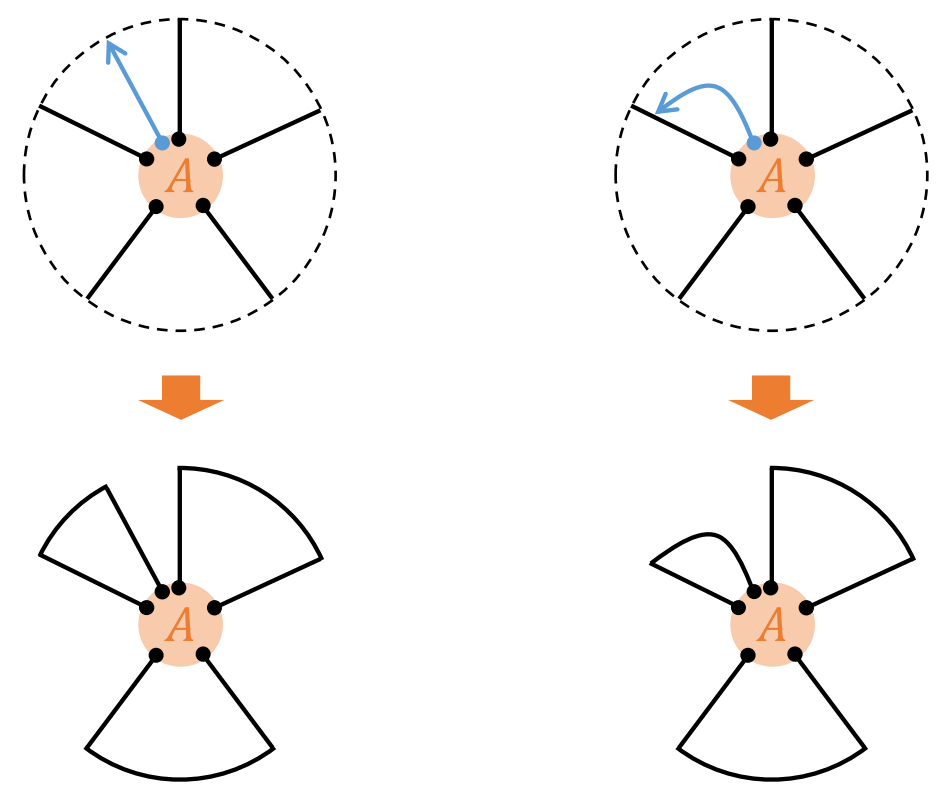

$t$ is contained in a half-integral cycle

$t$ is contained in a spoke

Figure 7: Augmentation by a single-segment augmenting path.

Proof of Lemma 2. Let $P=P_{1} \circ \cdots \circ P_{p}$ be the given $y$-augmenting path and $t=t(P)$. If $p>1$, by Corollary 4 , we obtain in linear time a basic $\mathcal{F}$-packing $y^{\prime}$ of the same size and a single-segment $y^{\prime}$ alternating path $P^{\prime}$ such that $t\left(P^{\prime}\right)=t$ and $P^{\prime} \equiv T_{y}(P)$. We now show that $P^{\prime}$ is a $y^{\prime}$-augmenting path. If $P$ satisfies the condition 1 or 2 of the $y$-augmenting paths (Definition 3$), P^{\prime}$ is a $y^{\prime}$ augmenting path because $t\left(P^{\prime}\right)=t$ is still contained in $A \backslash V\left(y^{\prime}\right)$ or $V\left(y^{\prime}\right) \backslash V_{1}\left(y^{\prime}\right)$. If $P$ satisfies the condition 3, the spoke $S$ that contains $t$ in $y$ might not exist in $y^{\prime}$. However, from the condition $3 \mathrm{~b}$, $t$ is still contained in a spoke $S^{\prime}$ in $y^{\prime}$, which may not be identical to $S$. By the same argument as in the proof of the second statement of Lemma 9, we have $B_{y^{\prime}}(t) \equiv B_{y}(t)$. Therefore, we have $T_{y^{\prime}}\left(P^{\prime}\right)=P^{\prime} \equiv T_{y}(P) \not \equiv B_{y}(t) \equiv B_{y^{\prime}}(t)$. Thus, $P^{\prime}$ is a $y^{\prime}$-augmenting path.

We can now concentrate on the case when $p=1$ (Figure 7). If the condition 1 is satisfied, $P$ is a conflicting path containing no vertices in $V(y)$. Therefore, we can obtain a basic $\mathcal{F}$-packing of size $|y|+1$ by inserting the integral path $P$. Otherwise, let $\left(\left\{S_{1}, \ldots, S_{d}\right\}, H_{1} \circ \cdots \circ H_{d}\right)$ be the wheel containing $t$. W.l.o.g., we can assume that $t$ is contained in $H_{d}$ or $S_{d}$.

If $t$ is contained in $H_{d}$, let $F$ be the prefix subpath of $H_{d}$ to $t$ and $B$ be the suffix subpath of $H_{d}$ from $t$ (i.e., $F \circ B=H_{d}$ ). We have $S_{d} \circ F \not \equiv S_{1} \circ B^{-1}$ because $S_{d} \circ H_{d} \circ S_{1}^{-1} \in \mathcal{F}$ Thus, at least one of $S_{d} \circ F \circ P^{-1}$ and $S_{1} \circ B^{-1} \circ P^{-1}$ is in $\mathcal{F}$, and w.l.o.g., we can assume the former case. Then, we can obtain a basic $\mathcal{F}$-packing of size $|y|+\frac{1}{2}$ by decomposing the wheel into $(d-1) / 2$ integral paths $\left\{S_{1} \circ H_{1} \circ S_{2}^{-1}, S_{3} \circ H_{3} \circ S_{4}^{-1}, \ldots, S_{d-2} \circ H_{d-2} \circ S_{d-1}^{-1}\right\}$ and by inserting an integral path $S_{d} \circ F \circ P^{-1}$.

If $t$ is contained in $S_{d}$, we have $P \not \equiv B(t)$. Then, we can obtain a basic $\mathcal{F}$-packing of size $|y|+\frac{1}{2}$ by decomposing the wheel into $(d-1) / 2$ integral paths $\left\{S_{1} \circ H_{1} \circ S_{2}^{-1}, S_{3} \circ H_{3} \circ S_{4}^{-1}, \ldots, S_{d-2} \circ\right.$ $\left.H_{d-2} \circ S_{d-1}^{-1}\right\}$ and by inserting an integral path $P \circ B(t)^{-1}$. 


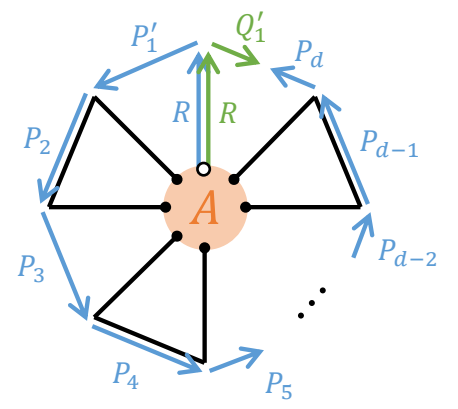

(a)
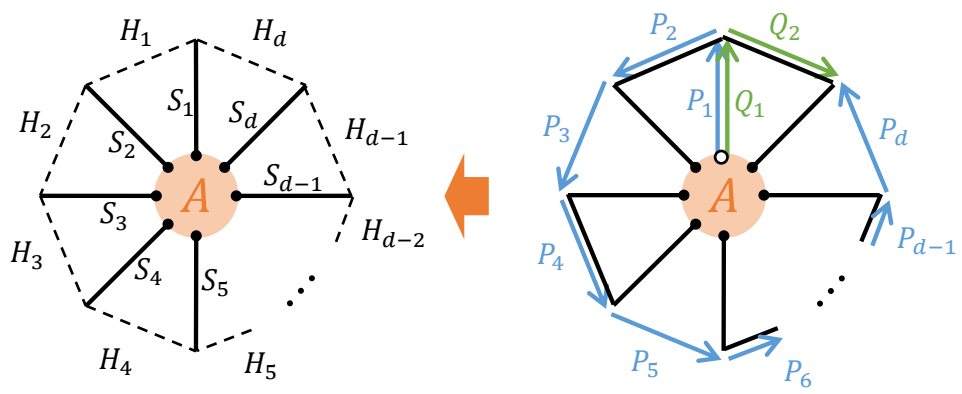

(b)

Figure 8: Applying Lemma 10.

\subsubsection{Augmentation by Augmenting Pair}

This subsection aims to prove Lemma 3.

Intuitively, we want to augment $y$ as follows. We first simplify the common prefix $R$ of $(P, Q)$ by applying Corollary 3, and then obtain a new wheel whose half-integral cycle is $P^{\prime} \circ Q^{\prime-1}$. However, it is not that easy. This approach does not work when $P^{\prime}$ or $Q^{\prime}$ intersects with spokes or intersects with the same integral path multiple times. Therefore, we augment $y$ by gradually simplifying the augmenting pair. First, we prove the lemma against a special case.

Lemma 10. Given a basic $\mathcal{F}$-packing $y$ and a y-augmenting pair $\left(P=P_{1} \circ \cdots \circ P_{p}, Q=Q_{1} \circ \cdots \circ Q_{q}\right)$ satisfying all the following conditions, a basic $\mathcal{F}$-packing of size $|y|+\frac{1}{2}$ can be constructed in linear time.

1. None of the $P_{i}$ 's are contained in the spokes.

2. None of the integral paths contain multiple $P_{i}$ 's.

3. One of the following two conditions is satisfied:

(a) $p \geq q=1$ or

(b) $p \geq q=2, P_{1}=Q_{1}$, and $P_{2}$ and $Q_{2}$ are contained in an integral path in the opposite direction.

Proof. When $(P, Q)$ satisfies the condition $3 \mathrm{a}$, we further divide the case into the following two cases: $p=1$ or $p \geq 2$.

If $p=1,(P, Q)$ is a single-branching pair such that $P \not \equiv Q$. Therefore, we can obtain a basic $\mathcal{F}$-packing of size $|y|+\frac{1}{2}$ by introducing a new wheel $P \circ Q^{-1}$ of degree one.

In $p \geq 2$, let $R$ be the common prefix and let us write $P_{1}=R \circ P_{1}^{\prime}$ and $Q_{1}=R \circ Q_{1}^{\prime}$. We set $d:=p+1$ and $P_{d}:=(t(P))$ if $p$ is even; otherwise, we set $d:=p$. We define paths $\left\{H_{1}, \ldots, H_{d}\right\}$ and $\left\{S_{1}, \ldots, S_{d}\right\}$ as follows (Figure $8(\mathrm{a})$ ).

- $H_{1}:=P_{1}^{\prime}$.

- $H_{i}:=P_{i}$ for $i \in\{2, \ldots, d-1\}$.

- $H_{d}:=P_{d} \circ Q_{1}^{-1}$. 
- $S_{1}:=R$.

- $S_{i}:=F\left(P_{i}\right)$ for even $i \in\{2,4, \ldots, d-1\}$.

- $S_{i}:=B\left(P_{i-1}\right)$ for odd $i \in\{3,5, \ldots, d\}$.

We now show that these paths form a wheel. The first condition of the wheel (Definition 1) is trivially satisfied. As none of the integral paths in $y$ contain multiple $P_{i}$ 's, the second and third conditions are satisfied. We can see that the fourth condition is satisfied as follows.

- $S_{1} \circ H_{1} \circ S_{2}^{-1}=R \circ P_{1}^{\prime} \circ F\left(P_{2}\right)^{-1}=P_{1} \circ F\left(P_{2}\right)^{-1} \in \mathcal{F}$.

- $S_{i} \circ H_{i} \circ S_{i+1}^{-1}=F\left(P_{i}\right) \circ P_{i} \circ B\left(P_{i}\right)^{-1} \in \mathcal{F}$ for even $i \in\{2,4, \ldots, d-1\}$.

- $S_{i} \circ H_{i} \circ S_{i+1}^{-1}=B\left(P_{i-1}\right) \circ P_{i} \circ F\left(P_{i+1}\right)^{-1} \in \mathcal{F}$ for odd $i \in\{3,5, \ldots, d-2\}$.

- $S_{d} \circ H_{d} \circ S_{1}^{-1}=B\left(P_{d-1}\right) \circ P_{d} \circ Q_{1}^{\prime-1} \circ R^{-1}=T(P) \circ T(Q)^{-1} \in \mathcal{F}$.

Thus, we can obtain a basic $\mathcal{F}$-packing of size $|y|+\frac{1}{2}$ by removing the $(d-1) / 2$ integral paths intersecting $P$ and by inserting the wheel of degree $d$.

Finally, we consider the case when $(P, Q)$ satisfies the condition $3 \mathrm{~b}$. Note that $p$ must be odd from the condition 3 of $y$-augmenting pairs (Definition 4). Let $d:=p$. We define paths $\left\{H_{1}, \ldots, H_{d}\right\}$ and $\left\{S_{1}, \ldots, S_{d}\right\}$ as follows (Figure $8(\mathrm{~b})$ ).

- $H_{i}:=P_{i+1}$ for $i \in\{1, \ldots, d-1\}$.

- $H_{d}:=Q_{2}^{-1}$.

- $S_{1}:=P_{1}$.

- $S_{i}:=B\left(P_{i}\right)$ for even $i \in\{2,4, \ldots, d-1\}$.

- $S_{i}:=F\left(P_{i+1}\right)$ for odd $i \in\{3,5, \ldots, d-2\}$.

- $S_{d}:=B\left(Q_{2}\right)$.

We now show that these paths form a wheel. The first condition of the wheel is trivially satisfied. The second and third conditions are satisfied because none of the integral paths in $y$ contain multiple $P_{i}^{\prime}$ 's. We can see that the fourth condition is satisfied as follows.

- $S_{1} \circ H_{1} \circ S_{2}^{-1}=P_{1} \circ P_{2} \circ B\left(P_{2}\right)^{-1}=P_{1} \circ\left(B\left(P_{2}\right) \circ P_{2}^{-1}\right)^{-1}=Q_{1} \circ F\left(Q_{2}\right)^{-1} \in \mathcal{F}$.

- $S_{i} \circ H_{i} \circ S_{i+1}^{-1}=B\left(P_{i}\right) \circ P_{i+1} \circ F\left(P_{i+2}\right)^{-1} \in \mathcal{F}$ for even $i \in\{2,4, \ldots, d-3\}$.

- $S_{i} \circ H_{i} \circ S_{i+1}^{-1}=F\left(P_{i+1}\right) \circ P_{i+1} \circ B\left(P_{i+1}\right)^{-1} \in \mathcal{F}$ for odd $i \in\{3,5, \ldots, d-2\}$.

- $S_{d-1} \circ H_{d-1} \circ S_{d}^{-1}=B\left(P_{d-1}\right) \circ P_{d} \circ B\left(Q_{2}\right)^{-1}=T(P) \circ T(Q)^{-1} \in \mathcal{F}$.

- $S_{d} \circ H_{d} \circ S_{1}^{-1}=B\left(Q_{2}\right) \circ Q_{2}^{-1} \circ P_{1}^{-1}=F\left(P_{2}\right) \circ P_{1}^{-1} \in \mathcal{F}$.

Thus, we can obtain a basic $\mathcal{F}$-packing of size $|y|+\frac{1}{2}$ by removing the $(d-1) / 2$ integral paths intersecting $P$ and by inserting the wheel of degree $d$. 


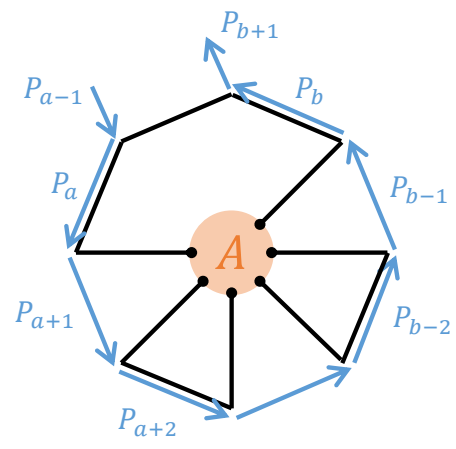

$y$

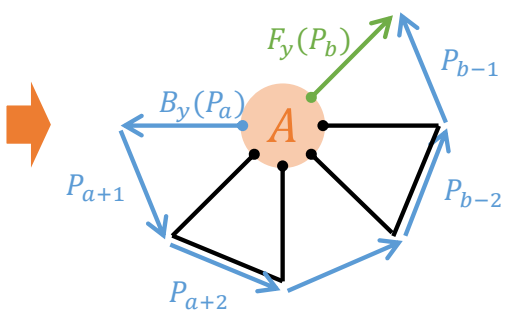

$y^{\prime}$
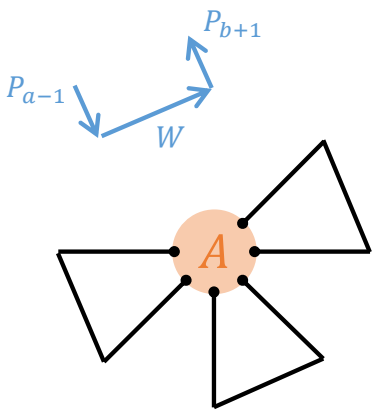

$\bar{y}$

Figure 9: Applying Lemma 11.

Next, we provide two lemmas for weakening the assumptions in the abovementioned lemma.

Lemma 11. Given a basic $\mathcal{F}$-packing y and a y-augmenting pair $\left(P=P_{1} \circ \cdots \circ P_{p}, Q=Q_{1} \circ \cdots \circ Q_{q}\right)$ satisfying all the following conditions, a basic $\mathcal{F}$-packing of size $|y|+\frac{1}{2}$ can be constructed in linear time.

1. None of the $P_{i}$ 's are contained in the spokes.

2'. Any two segments of $P$ contained in the same integral path have the same direction.

3. One of the following two conditions is satisfied:

(a) $p \geq q=1$ or

(b) $p \geq q=2, P_{1}=Q_{1}$, and $P_{2}$ and $Q_{2}$ are contained in an integral path in the opposite direction.

Proof. Note that the conditions 1 and 3 are the same as those in Lemma 10. Besides, if the condition $3 \mathrm{~b}$ is satisfied, from the condition $5 \mathrm{~b}$ of $y$-alternating paths (Definition 2), the integral path containing $P_{2}$ and $Q_{2}$ never contain any other segments. We call a segment of $P$ obstructive if it is contained in an integral path containing multiple segments of $P$. If there exist no obstructive segments, the condition 2 of Lemma 10 is satisfied. Thus, we can obtain a basic $\mathcal{F}$-packing of size $|y|+\frac{1}{2}$ by applying Lemma 10 . We repeat the following process while obstructive segments exist.

Let $P_{a}$ be the first obstructive segment and $P_{b}(b>a)$ be the next segment contained in the integral path containing $P_{a}$. From the condition $5 \mathrm{~b}$ of the $y$-alternating paths (Definition 2 ), $P_{b}$ is contained in $F\left(P_{a}\right)$, and $B\left(P_{a-2}\right) \circ P_{a-1} \equiv B\left(P_{a}\right) \circ P_{a}^{-1}$ holds. We construct a basic $\mathcal{F}$-packing $\bar{y}$ of the same size and a $\bar{y}$-augmenting pair $(\bar{P}, Q)$ such that the precondition of this lemma is satisfied and the number of obstructive segments strictly decreases as follows (see Figure 9).

First, we construct a basic $\mathcal{F}$-packing $y^{\prime}$ of size $|y|-1$ by removing the integral path containing $P_{a}$ and $P_{b}$ from $y$. Observe that $P^{\prime}:=\left(B_{y}\left(P_{a}\right) \circ P_{a+1}\right) \circ P_{a+2} \circ \cdots \circ P_{b-1}$ is a $y^{\prime}$-alternating path. Then, by applying Corollary 4 against $y^{\prime}$ and $P^{\prime}$, we obtain a basic $\mathcal{F}$-packing $y^{\prime \prime}$ of size $|y|-1$ and a single-segment $y^{\prime \prime}$-alternating path $P^{\prime \prime}$ satisfying $P^{\prime \prime} \equiv T_{y^{\prime}}\left(P^{\prime}\right)=B_{y^{\prime}}\left(P_{b-2}\right) \circ P_{b-1}=B_{y}\left(P_{b-2}\right) \circ P_{b-1}$. From the condition $5 \mathrm{~b}$ of the $y$-alternating path $P, B_{y}\left(P_{b-2}\right) \circ P_{b-1} \not \equiv F_{y}\left(P_{b}\right)$ holds. From the choice of $P_{b}, F_{y}\left(P_{b}\right)$ is internally disjoint from $P^{\prime \prime}$. Thus, we can obtain a basic $\mathcal{F}$-packing $\bar{y}$ of size $|y|$ 
by introducing a new integral path $P^{\prime \prime} \circ F_{y}\left(P_{b}\right)$. Let $W$ be the path from $s\left(P_{a}\right)$ to $t\left(P_{b}\right)$ along the integral path and let $\bar{P}:=P_{1} \circ \cdots \circ P_{a-2} \circ\left(P_{a-1} \circ W \circ P_{b+1}\right) \circ P_{b+2} \circ \cdots \circ P_{p}$.

Finally, we prove that $(\bar{P}, Q)$ is a $\bar{y}$-augmenting pair satisfying the preconditions of this lemma. From the construction of $\bar{y}$ and $\bar{P}$, the conditions $1-4$ of the $\bar{y}$-alternating paths (Definition 2) are clearly satisfied. The condition $5 \mathrm{~b}$ is satisfied because $B_{\bar{y}}\left(P_{a-2}\right) \circ\left(P_{a-1} \circ W \circ P_{b+1}\right) \equiv B_{y}\left(P_{a-2}\right) \circ$ $P_{a-1} \circ W \circ P_{b+1} \equiv B_{y}\left(P_{a}\right) \circ P_{a}^{-1} \circ W \circ P_{b+1} \equiv B_{y}\left(P_{b}\right) \circ P_{b+1}$ holds. Thus, $\bar{P}$ is a $\bar{y}$-alternating path. Because $T_{\bar{y}}(\bar{P}) \equiv T_{y}(P) \neq T_{y}(Q) \equiv T_{\bar{y}}(Q)$ holds and $(\bar{P}, Q)$ satisfies the precondition 3 of the lemma, $(\bar{P}, Q)$ is a $\bar{y}$-augmenting pair. Because no segments of $\bar{P}$ are newly contained in the spokes in $\bar{y}$, the condition 1 of the lemma is satisfied. From the choice of $P_{a}$, for any even $i \in\{a+2, \ldots, b-2\}, B_{y^{\prime}}\left(P_{i}\right)$ contains no segments from $\left\{P_{2}, \ldots, P_{a-2}\right\}$. Therefore, no two segments of $\bar{P}$ are newly contained in a same integral path in $\bar{y}$. Thus, the condition 2' is satisfied. When $(P, Q)$ satisfies the condition $3 \mathrm{a},(\bar{P}, Q)$ also satisfies the condition $3 \mathrm{a}$. When $(P, Q)$ satisfies the condition $3 \mathrm{~b},(\bar{P}, Q)$ also satisfies the condition $3 \mathrm{~b}$ because the integral path containing $P_{2}$ and $Q_{2}$ remains in $\bar{y}$. Thus, all the conditions in the lemma are satisfied.

We can find the pair $\left(P_{a}, P_{b}\right)$ by gradually increasing an index $i$, which is not reset during the repetition, and by searching for $P_{j}$ contained in $F\left(P_{i}\right)$ by traversing the integral path. Therefore, each edge is traversed at most once through the whole process. Thus, the total running time is linear in the graph size.

Lemma 12. Given a basic $\mathcal{F}$-packing y and a y-augmenting pair $\left(P=P_{1} \circ \cdots \circ P_{p}, Q=Q_{1} \circ \cdots \circ Q_{q}\right)$, either of a y-augmenting path or a y-augmenting pair $(\bar{P}, \bar{Q})$ satisfying all the following conditions can be constructed in linear time.

1. All the segments of $\bar{Q}$, except for the last one, are contained in $\bar{P}$.

2. $\bar{P}$ can be written as $\bar{P}=P \circ Q_{q}^{-1} \circ Q_{q-1}^{-1} \circ \cdots \circ Q_{q^{\prime}}^{-1}$ for some $q^{\prime}$.

3. The common prefix of $(\bar{P}, \bar{Q})$ contains the common prefix of $(P, Q)$.

4. The following two conditions are satisfied for the new segments $S:=\left\{Q_{q}^{-1}, \ldots, Q_{q^{\prime}}^{-1}\right\}$ of $\bar{P}$ :

(a) no segments in $S$ are contained in the spokes, and

(b) any two segments in $S$ contained in the same integral path have the same direction.

Proof. Initially, the conditions 2-4 are trivially satisfied. We repeat the following process, which preserves these conditions. The condition 1 is satisfied when $q$ becomes one or $Q_{q-1}$ gets contained in $P$.

(Case 1) If both of $p$ and $q$ are odd, we update $P^{\prime} \leftarrow P_{1} \circ \cdots \circ P_{p-1} \circ\left(P_{p} \circ Q_{q}^{-1}\right)$ and $Q^{\prime} \leftarrow$ $Q_{1} \circ \cdots \circ Q_{q-1}$. Because $P_{p}$ and $Q_{q}$ share no edges and because $T\left(P^{\prime}\right) \circ T\left(Q^{\prime}\right)^{-1}=\left(B\left(P_{p-1}\right) \circ\right.$ $\left.\left(P_{p} \circ Q_{q}^{-1}\right)\right) \circ B\left(Q_{q-1}\right)^{-1}=\left(B\left(P_{p-1}\right) \circ P_{p}\right) \circ\left(B\left(Q_{q-1}\right) \circ Q_{q}\right)^{-1}=T(P) \circ T(Q)^{-1}$ holds, $\left(P^{\prime}, Q^{\prime}\right)$ is a $y$-augmenting pair.

(Case 2) If $p$ is even and $q$ is odd, we update $P^{\prime} \leftarrow P_{1} \circ \cdots \circ P_{p-1} \circ P_{p} \circ Q_{q}^{-1}$ and $Q^{\prime} \leftarrow Q_{1} \circ \cdots \circ Q_{q-1}$. Because $P_{p}$ and $Q_{q}$ share no edges and because $T\left(P^{\prime}\right) \circ T\left(Q^{\prime}\right)^{-1}=\left(B\left(P_{p}\right) \circ Q_{q}^{-1}\right) \circ B\left(Q_{q-1}\right)^{-1}=$ $B\left(P_{p}\right) \circ\left(B\left(Q_{q-1}\right) \circ Q_{q}\right)^{-1}=T(P) \circ T(Q)^{-1}$ holds, $\left(P^{\prime}, Q^{\prime}\right)$ is a $y$-augmenting pair. 

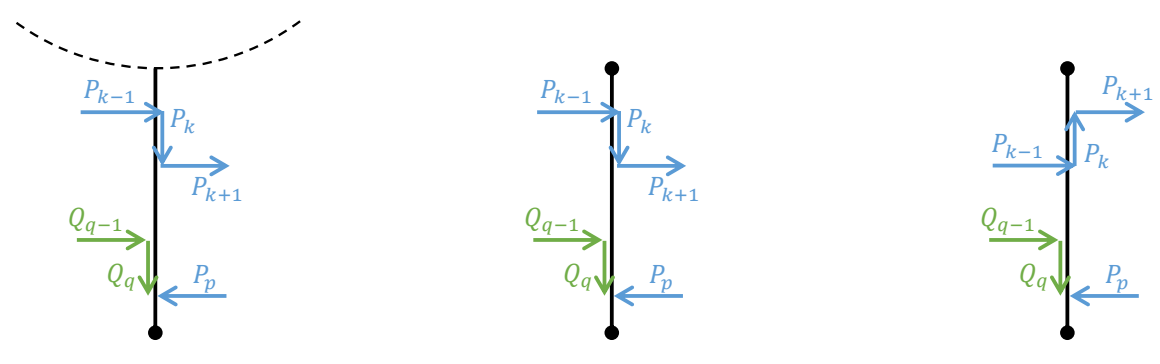

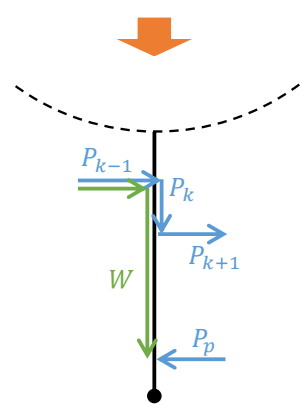

(Case 3)

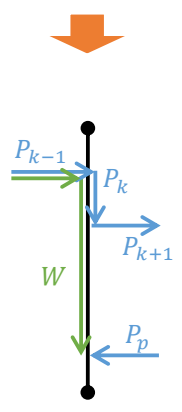

(Case $4(1))$

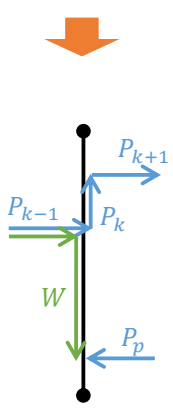

(Case $4(2))$

Figure 10: Applying Lemma 12.

(Case 3) If $p$ is odd, $q$ is even, and $Q_{q}$ is contained in a spoke, then we search for the nearest segment of $P$ contained in $F\left(Q_{q}\right)$ by traversing the spoke. Note that none of the other segments of $Q$ are contained in $F\left(Q_{q}\right)$ from the condition 5 c of the $y$-alternating paths (Definition 2). Moreover, each segment of $P$ is fully contained in $F\left(Q_{q}\right)$ or internally disjoint from $F\left(Q_{q}\right)$ because $Q_{q}$ shares no edges with $P$. Thus, each edge is traversed at most once through the entire process, and the total running time of this part is linear in the graph size. If none of the $P_{i}$ 's are contained in $F\left(Q_{q}\right)$, then $P$ is a $y$-augmenting path because $t(P)$ is contained in the spoke, and $T(P) \not \equiv T(Q)=B(t(P))$ holds. Otherwise, let $P_{k}$ be the nearest segment of $P$ contained in $F\left(Q_{q}\right)$; let $W$ be the path from $s\left(P_{k}\right)$ to $t(P)$ along the spoke; and let $\bar{Q}:=P_{1} \circ \cdots \circ P_{k-1} \circ W$ (Figure 10 (left)). Because $T(\bar{Q})=B(W)=B\left(Q_{q}\right)=T(Q)$ holds, $(P, \bar{Q})$ is a $y$-augmenting pair. Note that this finishes the process.

(Case 4) If $p$ is odd, $q$ is even, and $Q_{q}$ is contained in an integral path, then we search for the nearest segment of $P$ or $Q$ contained in $F\left(Q_{q}\right)$ by traversing the integral path. Because each edge is traversed at most twice (in two directions) through the entire process, the total running time of this part is linear in the graph size. Note that from the condition $5 \mathrm{~b}$ of the $y$-alternating path $Q$ (Definition 2), $F\left(Q_{q}\right)$ cannot contain a segment $Q_{k}$ such that $Q_{k}$ has the same direction as $Q_{q}$ or has the opposite direction as $Q_{q}$ and $B\left(Q_{k-2}\right) \circ Q_{k-1} \not \equiv B\left(Q_{k}\right) \circ Q_{k}^{-1}$ holds.

If the nearest segment is $P_{k}$ such that $P_{k}$ has the same direction as $Q_{q}$ or has the opposite direction as $Q_{q}$ and $B\left(P_{k-2}\right) \circ P_{k-1} \not \equiv B\left(P_{k}\right) \circ P_{k}^{-1}$ holds, let $W$ be the path from $s\left(P_{k}\right)$ to $t(P)$ along the integral path and $\bar{Q}:=P_{1} \circ \cdots \circ P_{k-1} \circ W$ (see Figure 10 (right)). Because $T(\bar{Q})=B(W)=B\left(Q_{q}\right)=T(Q)$ holds, $(P, \bar{Q})$ is a $y$-augmenting pair, and we then finish the process. 
Otherwise (i.e., (a) no segments are contained in $F\left(Q_{q}\right)$, (b) the nearest segment is $Q_{k}$ that has the opposite direction as $Q_{q}$ and $B\left(Q_{k-2}\right) \circ Q_{k-1} \equiv B\left(Q_{k}\right) \circ Q_{k}^{-1}$ holds, or (c) the nearest segment is $P_{k}$ that has the opposite direction as $Q_{q}$ and $B\left(P_{k-2}\right) \circ P_{k-1} \equiv B\left(P_{k}\right) \circ P_{k}^{-1}$ holds), we update $P^{\prime} \leftarrow P_{1} \circ \cdots \circ P_{p} \circ Q_{q}^{-1}$ and $Q^{\prime} \leftarrow Q_{1} \circ \cdots \circ Q_{q-1}$. Note that in this case, from the condition $5 \mathrm{~b}$ of the $y$-alternating paths (Definition 2) and the condition 4 of $y$-augmenting pairs (Definition 4), conditions (b) and (c) hold not only against the nearest segment $Q_{k}$ or $P_{k}$, but also against any segments contained in $F\left(Q_{q}\right)$.

First, we prove that $P^{\prime}$ is a $y$-alternating path. The first four conditions of the $y$-alternating paths (Definition 2) are clearly satisfied. The condition 5 is satisfied for $i=p+1$ because $B\left(P_{p-1}\right) \circ$ $P_{p}=T(P) \not \equiv T(Q)=B\left(Q_{q}\right)=F\left(Q_{q}^{-1}\right)$ holds. For checking the condition 5 against the other $P_{i}$ 's, it suffices to show that for any segment $P_{i}$ contained in the same integral path as $Q_{q}$, it holds that $B\left(P_{i-2}\right) \circ P_{i-1} \equiv B\left(P_{i}\right) \circ P_{i}^{-1}$, and $Q_{q}$ is contained in $F\left(P_{i}\right)$. Let $P_{i}$ be a segment contained in $B\left(Q_{q}\right)$. From the condition 4 of the $y$-augmenting pairs (Definition 4 ), $P_{i}$ has the same direction as $Q_{q}$. Thus, $Q_{q}$ is contained in $F\left(P_{i}\right)$. Therefore, from the condition 4 again, $B\left(P_{i-2}\right) \circ P_{i-1} \equiv B\left(P_{i}\right) \circ P_{i}^{-1}$ holds. Let $P_{i}$ be a segment contained in $F\left(Q_{q}\right)$. As we have discussed earlier, $B\left(P_{i-2}\right) \circ P_{i-1} \equiv B\left(P_{i}\right) \circ P_{i}^{-1}$ then holds, and $P_{i}$ has the opposite direction as $Q_{q}$, implying that $Q_{q}$ is contained in $F\left(P_{i}\right)$.

Next, we prove that $\left(P^{\prime}, Q^{\prime}\right)$ is a $y$-augmenting pair. Conditions 1 and 3 of the $y$-augmenting pairs (Definition 4) are clearly satisfied. Because $T\left(P^{\prime}\right)=B\left(Q_{q}^{-1}\right)=F\left(Q_{q}\right) \not \equiv B\left(Q_{q-2}\right) \circ Q_{q-1}=$ $T\left(Q^{\prime}\right)$ holds, the condition 2 is satisfied. For checking the condition 4, it suffices to show that (1) none of the $Q_{j}$ 's with $j<q$ are contained in $B\left(Q_{q}^{-1}\right)=F\left(Q_{q}\right)$ in the same direction as $Q_{q}$; (2) if $B\left(P_{p-1}\right) \circ P_{p} \not \equiv B\left(Q_{q}^{-1}\right) \circ\left(Q_{q}^{-1}\right)^{-1}=F\left(Q_{q}\right) \circ Q_{q}$, none of the $Q_{j}$ 's with $j<q$ are contained in $F\left(Q_{q}^{-1}\right)=B\left(Q_{q}\right)$ in the opposite direction as $Q_{q} ;(3)$ for any $Q_{j}$ contained in an integral path, $Q_{q}$ is not contained in $B\left(Q_{j}\right)$ in the same direction as $Q_{j}$; and (4) if $B\left(Q_{j-2}\right) \circ Q_{j-1} \not \equiv B\left(Q_{j}\right) \circ Q_{j}^{-1}$ holds, $Q_{q}$ is not contained in $F\left(Q_{j}\right)$ in the opposite direction as $Q_{j}$. All these conditions directly follow from the condition $5 \mathrm{~b}$ of the $y$-alternating path $Q$ (Definition 2).

Finally, we prove that $\left(P^{\prime}, Q^{\prime}\right)$ satisfies the conditions $2-4$ of this lemma. Conditions 2 and 3 are clearly satisfied. From the condition $5 \mathrm{~b}$ of the initial $y$-alternating path $Q, B\left(Q_{q}\right)$ contains none of the new segments $S$. Thus, if a segment in $S$ is contained in the same integral path as $Q_{q}$, it must be contained in $F\left(Q_{q}\right)$. Therefore, it has the same direction as $Q_{q}^{-1}$.

Finally, we prove Lemma 3 by combining Lemmas 11 and 12 .

Proof of Lemma 3. First, we apply Lemma 12 against $(Q, P)$. If we obtain a $y$-augmenting path, we obtain a basic $\mathcal{F}$-packing of size $|y|+\frac{1}{2}$ by applying Lemma 2. Otherwise, we obtain an updated $y$-augmenting pair $(\bar{Q}, \bar{P})$ such that all the segments of $\bar{P}$, except for the last one, are contained in the common prefix.

Next, we apply Lemma 12 against $(\bar{P}, \bar{Q})$. We finish if we obtain a $y$-augmenting path; otherwise, we obtain an updated $y$-augmenting pair $(\hat{P}, \hat{Q})$. Let $\hat{P}:=\hat{P}_{1} \circ \cdots \circ \hat{P}_{\hat{p}}$ and $\hat{Q}:=\hat{Q}_{1} \circ \cdots \circ \hat{Q}_{\hat{q}}$. Then, $\hat{Q}_{\hat{q}-1}$ is contained in $\hat{P}_{\hat{q}-1}$. Note that this implies that $\hat{P}_{i}=\hat{Q}_{i}$ holds for any $i \in\{1, \ldots, \hat{q}-2\}$. Moreover, $\hat{P}_{\hat{q}-1}=\hat{Q}_{\hat{q}-1}$ also holds if $\hat{q}$ is even. We have $\hat{q} \geq \bar{p}-1$ because the common prefix of $(\hat{P}, \hat{Q})$ contains the common prefix of $(\bar{P}, \bar{Q})$ and because $\hat{P}_{\bar{p}-1}$ is contained in the common prefix of $(\bar{P}, \bar{Q})$. Let us assume that $\hat{q}=\bar{p}-1$ holds, and $\hat{q}$ is odd. In this case, $\hat{P}_{\hat{q}}$ is contained in $\hat{Q}_{\hat{q}}$. Therefore, $\hat{P}_{\hat{q}}=\hat{Q}_{\hat{q}}$ holds. Then, from the condition 3 of the $y$-augmenting pair $(\hat{P}, \hat{Q})$ (Definition 4), $\hat{p}$ must be even, and $\hat{P}_{\hat{p}}$ must be contained in the same integral path as $\hat{P}_{\bar{p}}$. However, 
this violates the condition $5 \mathrm{~b}$ of the $y$-alternating path $\hat{P}$ because $B\left(\hat{P}_{\bar{p}-2}\right) \circ \hat{P}_{\bar{p}-1}=T(\hat{Q}) \not \equiv T(\hat{P})=$ $B\left(\hat{P}_{\hat{p}}\right)=B\left(\hat{P}_{\bar{p}}\right) \circ \hat{P}_{\bar{p}}^{-1}$ holds. Thus, $\hat{q} \geq \bar{p}$ holds if $\hat{q}$ is odd. We consider two cases.

If $\hat{q}$ is odd or $\hat{P}_{\hat{q}}$ and $\hat{Q}_{\hat{q}}$ have the same direction, let $r$ be the maximum even integer at most $\hat{q}$. We apply Corollary 3 independently against $\hat{P}$ and $\hat{Q}$ and obtain a basic $\mathcal{F}$-packing $y^{\prime}$ of the same size and $y^{\prime}$-alternating paths $P^{\prime}$ and $Q^{\prime}$. Here, $\hat{P}_{i}=\hat{Q}_{i}$ holds for any $i \in\{1, \ldots, r-1\}$, and $F\left(\hat{P}_{r}\right)=F\left(\hat{Q}_{r}\right)$ holds. Hence, the basic $\mathcal{F}$-packing obtained by these two applications are the same. We then, thus, use the same symbol $y^{\prime}$. Because $T_{y^{\prime}}\left(P^{\prime}\right) \equiv T_{y}(\hat{P}) \not \equiv T_{y}(\hat{Q}) \equiv T_{y^{\prime}}\left(Q^{\prime}\right)$ holds, $\left(P^{\prime}, Q^{\prime}\right)$ is a $y^{\prime}$-augmenting pair. We now show that $\left(P^{\prime}, Q^{\prime}\right)$ satisfies the conditions in Lemma 11 . Because $Q^{\prime}$ is single-segment, the third condition is satisfied. We have $r+2=\hat{q}+1>\bar{p}$ when $\hat{q}$ is odd, and we have $r+2=\hat{q}+2>\bar{p}$ when $\hat{q}$ is even. Therefore, from the condition 4 of Lemma 12, the first and the second conditions hold. Thus, we obtain a basic $\mathcal{F}$-packing of size $|y|+\frac{1}{2}$ by applying Lemma 11 against $\left(P^{\prime}, Q^{\prime}\right)$.

If $\hat{q}$ is even and $\hat{P}_{\hat{q}}$ and $\hat{Q}_{\hat{q}}$ have the opposite direction, let $r:=\hat{q}-2$. We apply Corollary 3 against independently $\hat{P}$ and $\hat{Q}$ and obtain a basic $\mathcal{F}$-packing $y^{\prime}$ of the same size and $y^{\prime}$-alternating paths $P^{\prime}$ and $Q^{\prime}$. Here, $\hat{P}_{i}=\hat{Q}_{i}$ holds for any $i \in\{1, \ldots, r\}$. Hence, the basic $\mathcal{F}$-packings obtained by these two applications are the same. We, thus, use the same symbol $y^{\prime}$. Because $T_{y^{\prime}}\left(P^{\prime}\right) \equiv T_{y}(\hat{P}) \not \equiv T_{y}(\hat{Q}) \equiv T_{y^{\prime}}\left(Q^{\prime}\right)$ holds, $\left(P^{\prime}, Q^{\prime}\right)$ is a $y^{\prime}$-augmenting pair. We now show that $\left(P^{\prime}, Q^{\prime}\right)$ satisfies the conditions in Lemma 11. Because we set $r:=\hat{q}-2$, the number of segments of $Q^{\prime}$ is two. Because $\hat{P}_{\hat{q}-1}=\hat{Q}_{\hat{q}-1}$ holds, the first segments of $P^{\prime}$ and $Q^{\prime}$ are the same. Because $\hat{P}_{\hat{q}}$ and $\hat{Q}_{\hat{q}}$ have the opposite direction, the second segments of $P^{\prime}$ and $Q^{\prime}$ have the opposite direction. Thus, the third condition is satisfied. Note that in this case, $\hat{P}_{\hat{q}}$ and $\hat{Q}_{\hat{q}}$ are contained in an integral path and, from the condition $5 \mathrm{~b}$ of the $y$-alternating paths (Definition 2), the integral path containing $\hat{P}_{\hat{q}}$ and $\hat{Q}_{\hat{q}}$ never contain any other segments. Moreover, because $r+4=\hat{q}+2>\bar{p}$, the first and second conditions follow from the condition 4 of Lemma 12. Thus, we obtain a basic $\mathcal{F}$-packing of size $|y|+\frac{1}{2}$ by applying Lemma 11 against $\left(P^{\prime}, Q^{\prime}\right)$.

\section{Farthest Cover}

This section provides an algorithm for computing a farthest minimum half-integral $\mathcal{F}$-cover and proves the following theorem.

Theorem 3. Let $C$ be a set of $0 / 1 /$ all constraints on variables $V$ and $\varphi_{A}$ be a partial assignment for a subset $A \subseteq V$. Given the primal graph of $C$, the set $A$, an incremental-test oracle for $\left(C, \varphi_{A}\right)$, and an integer $k$, we can compute a pair of a farthest minimum half-integral $\mathcal{F}_{C, \varphi_{A}}$-cover $x$ and a maximum half-integral $\mathcal{F}_{C, \varphi_{A}}$-packing y with $|x|=|y| \leq \frac{k}{2}$ or correctly conclude that the size of the minimum half-integral $\mathcal{F}_{C, \varphi_{A}}$-cover is at least $\frac{k+1}{2}$ in $O(k m T)$ time, where $m$ is the number of constraints, and $T$ is the running time of the incremental-test oracle.

We use the following structure of a minimum half-integral $\mathcal{F}$-cover.

Lemma 13. The following holds for any maximum basic $\mathcal{F}$-packing $y$ and any minimum halfintegral $\mathcal{F}$-cover $x$.

1. $x(V(I))=1$ for any integral path $I$ of $y$.

2. $x(V(S))=\frac{1}{2}$ for any spoke $S$ of $y$. 
Proof. For a wheel $W$, we denote the degree of $W$ by $d(W)$ and the set of vertices contained in $W$ by $V(W)$. Note that $V(W)$ is not a multiset. First, we prove that $x(V(W))$ is always at least $\frac{d(W)}{2}$ and if the equality holds, then $x(V(S))=\frac{1}{2}$ holds for any spoke $S$ of $W$. Let $W$ be a wheel with a halfintegral cycle $H_{1} \circ \cdots \circ H_{d}$ and spokes $\left\{S_{1}, \ldots, S_{d}\right\}$. We define $H:=V(W) \backslash\left(V\left(S_{1}\right) \cup \ldots \cup V\left(S_{d}\right)\right)$. We then have

$$
\begin{aligned}
x(V(W)) & =x(H)+\sum_{i=1}^{d} x\left(V\left(S_{i}\right)\right) \\
& =\frac{1}{2} x(H)+\frac{1}{2} \sum_{i=1}^{d} x\left(V\left(S_{i} \circ H_{i} \circ S_{i+1}^{-1}\right)\right) \\
& \geq \frac{1}{2} \sum_{i=1}^{d} 1 \\
& =\frac{d}{2} .
\end{aligned}
$$

If $x(V(W))=\frac{d}{2}$ holds, we have $x(H)=0$ and $x\left(V\left(S_{i}\right)\right)+x\left(V\left(S_{i+1}\right)\right)=1$ for any $i \in\{1, \ldots, d\}$. Because $d$ is odd, this implies that $x\left(V\left(S_{i}\right)\right)=\frac{1}{2}$ holds for any $i \in\{1, \ldots, d\}$.

We now prove the lemma. Because all the integral paths and wheels do not share any vertices, we have

$$
\begin{aligned}
|x| & \geq \sum_{I: \text { integral path }} x(V(I))+\sum_{W: \text { wheel }} x(V(W)) \\
& \geq \sum_{I: \text { integral path }} 1+\sum_{W: \text { wheel }} \frac{d(W)}{2} \\
& =|y| \\
& =|x| .
\end{aligned}
$$

Therefore, $x(V(I))=1$ and $x(V(W))=\frac{d(W)}{2}$ must hold for any integral path $I$ and any wheel $W$.

Fix an arbitrary maximum basic $\mathcal{F}$-packing. From the above lemma, for every minimum halfintegral $\mathcal{F}$-cover $x$, we can construct unique indices $a_{x}$ and $b_{x}$ satisfying the following.

1. For every integral path $I=\left(v_{0}, \ldots, v_{\ell}\right), x\left(v_{a_{x}(I)}\right) \geq \frac{1}{2}, x\left(v_{b_{x}(I)}\right) \geq \frac{1}{2}$ and $a_{x}(I) \leq b_{x}(I)$ hold.

2. For every spoke $S=\left(v_{0}, \ldots, v_{\ell}\right), x\left(v_{a_{x}(S)}\right)=\frac{1}{2}$ holds.

Therefore, we obtain the following corollary.

Corollary 5. A minimum half-integral $\mathcal{F}$-cover $x^{\prime}$ dominates a minimum half-integral $\mathcal{F}$-cover $x$ if and only if the following conditions hold.

1. For every integral path $I, a_{x}(I) \leq a_{x^{\prime}}(I) \leq b_{x^{\prime}}(I) \leq b_{x}(I)$ holds.

2. For every spoke $S, a_{x}(S) \leq a_{x^{\prime}}(S)$ holds. 


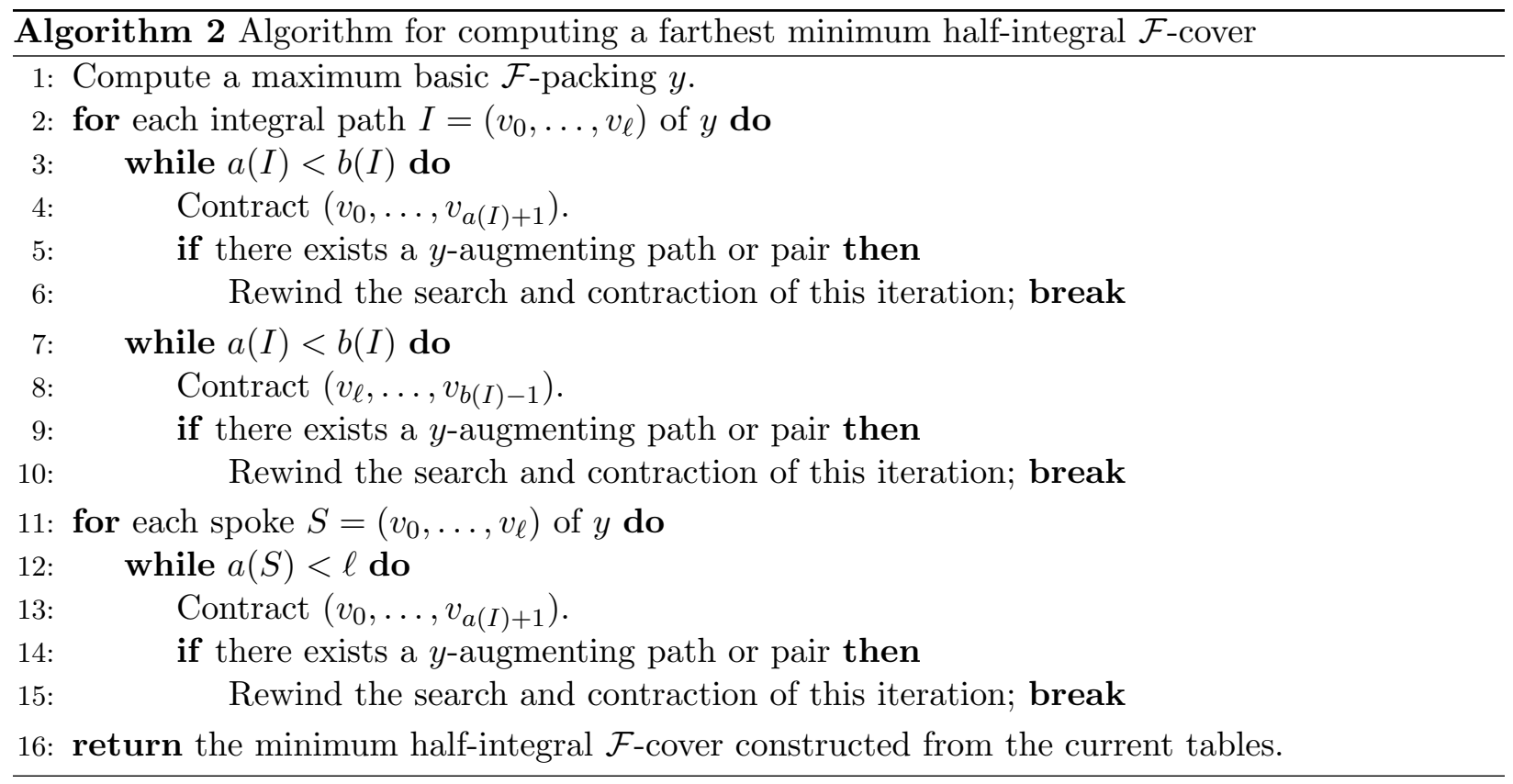

We use the following operation in our algorithm. Let $P$ be an implicational walk ending at a vertex $t$. First, we create a new vertex $t^{\prime}$ and introduce a constraint $\varphi(t)=\varphi\left(t^{\prime}\right)$ (along with a new edge $\left.t t^{\prime}\right)$. We then insert $t^{\prime}$ into $A$ and set $\varphi_{A}\left(t^{\prime}\right)=\operatorname{imp}(P)$. We call this operation as contracting $P$.

Lemma 14. Let $P$ be an implicational walk ending at a vertex $t$ and let $\mathcal{F}^{\prime}$ be the set of conflicting walks after contracting $P$. Then, any half-integral $\mathcal{F}^{\prime}$-cover $x$ is also a half-integral $\mathcal{F}$-cover, and any half-integral $\mathcal{F}$-cover $x$ satisfying $x(V(P) \backslash\{t\})=0$ is also a half-integral $\mathcal{F}^{\prime}$-cover.

Proof. The first claim is trivial because $\mathcal{F} \subseteq \mathcal{F}^{\prime}$. We now prove the second claim. Suppose that $x$ is not a half-integral $\mathcal{F}^{\prime}$-cover. Then, there exists a walk $W \in \mathcal{F}^{\prime}$ with $x(V(W))<1$. Because $x$ is a half-integral $\mathcal{F}$-cover, $W$ must contain the edge $t^{\prime} t$. Therefore, we can write $W=t^{\prime} t \circ Q^{-1}$ for some implicational walk $Q$ with $\operatorname{imp}(Q) \neq \operatorname{imp}(P)$. We then have $x\left(V\left(P \circ Q^{-1}\right)\right)=x(V(W))<1$ and $P \circ Q^{-1} \in \mathcal{F}$, which contradicts the fact that $x$ is a half-integral $\mathcal{F}$-cover.

We now describe the algorithm for computing a farthest minimum half-integral $\mathcal{F}$-cover (see Algorithm 2). We iteratively apply the contraction in the algorithm. We denote the current set of the conflicting walks by $\mathcal{F}$ and the original set by $\mathcal{F}_{\text {orig. }}$. First, we compute a maximum basic $\mathcal{F}$-packing $y$ using the algorithm in Section 3. We keep and reuse the tables $(a, b$, prev, and tail) used in the last execution of Algorithm 1, which returned NO. We process the integral paths and the spokes of $y$ one by one, whose detail will be described later, by preserving the following invariants.

Lemma 15. The following invariants hold at any step of Algorithm 2.

1. y is a maximum basic $\mathcal{F}$-packing.

2. Let $x$ be the minimum half-integral $\mathcal{F}$-cover constructed from the current tables as described in Section 3.3 (i.e., $a_{x}=a$ and $b_{x}=b$ hold). Then, any minimum half-integral $\mathcal{F}_{\text {orig-cover }}$ dominating $x$ is also a minimum half-integral $\mathcal{F}$-cover dominating $x$. 
3. For any processed integral path $I$, there exists no minimum half-integral $\mathcal{F}$-cover $x^{\prime}$ satisfying $a(I)<a_{x^{\prime}}(I) \leq b_{x^{\prime}}(I) \leq b(I)$ or $a(I) \leq a_{x^{\prime}}(I) \leq b_{x^{\prime}}(I)<b(I)$.

4. For any processed spoke $S$, there exists no minimum half-integral $\mathcal{F}$-cover $x^{\prime}$ satisfying $a(S)<$ $a_{x^{\prime}}(S)$.

When all the integral paths and the spokes are processed, we return the minimum half-integral $\mathcal{F}$-cover $x$ constructed from the current tables. We can easily prove the correctness of the algorithm from these invariants.

Lemma 16. When all the integral paths and the spokes are processed, the minimum half-integral $\mathcal{F}$-cover $x$ of $G$ constructed from the current tables is a farthest minimum half-integral $\mathcal{F}_{\text {orig-cover. }}$

Proof. From the invariants 3 and 4 and Corollary $5, x$ is a farthest minimum half-integral $\mathcal{F}$-cover. Because $\mathcal{F}_{\text {orig }} \subseteq \mathcal{F}$ and from the invariant 1, $x$ is also a minimum half-integral $\mathcal{F}_{\text {orig-cover. Suppose }}$ that there exists a minimum half-integral $\mathcal{F}_{\text {orig-cover }} x^{\prime}$ dominating $x$. Then, from the invariant 2 , $x^{\prime}$ is a minimum half-integral $\mathcal{F}$-cover dominating $x$, which contradicts the fact that $x$ is a farthest minimum half-integral $\mathcal{F}$-cover.

We now describe how to process the integral paths and spokes. For each integral path $I=$ $\left(v_{0}, \ldots, v_{\ell}\right)$, we first repeat the following while $a(I)<b(I)$ holds (lines 3-6). Let $i:=a(I)+1$. We first contract the implicational path $\left(v_{0}, \ldots, v_{i}\right)$. When using the incremental-test oracle, this operation can be done in a constant time by setting $\mathcal{I}\left(v_{i}^{\prime}\right)=\mathcal{A}^{*}\left(\left(v_{0}, \ldots, v_{i}\right)\right)$, which has been precomputed. We then search for a $y$-augmenting path or pair using Algorithm 1. Instead of searching for a $y$-augmenting path/pair from scratch by initializing the tables, we restart the search from line 4 of Algorithm 1 by setting $s \leftarrow v_{i}^{\prime}$ and reusing the current tables. If the restarted search returns NO, we keep the tables updated by the restarted search and continue the repetition. Because $v_{i}^{\prime} v_{i} \not \equiv\left(v_{\ell}, \ldots, v_{i}\right)$ holds, we have $a(I) \geq i$ after the search. If the restarted search finds a $y$-augmenting path or pair, we rewind all the changes in this step (i.e., we restore the tables to the state before the search and remove the edge $v_{i}^{\prime} v_{i}$ inserted by the contraction), and then exit the repetition. Note that we do not rewind the changes in the previous steps where the restarted searches returned NO.

Claim 5. The restarted search can correctly compute a y-augmenting path or pair if exists.

Proof. We can virtually think as follows. Because the order of $A \backslash V(y)$ at line 3 of Algorithm 1 is arbitrary, we can choose completely the same order as the one used in the last failed search that has constructed the current tables. Note that, from the invariant $3 \mathrm{~b}$ in Lemma $5, v_{i}$ was not visited in the last execution. Therefore, the insertion of the edge $v_{i}^{\prime} v_{i}$ does not affect the search at all. In the end, the execution reaches to the final iteration of the while loop with $s=v_{i}^{\prime}$, and all the tables are completely the same as the current tables. Thus, instead of running Algorithm 1 from scratch, we can use the restarted search.

Claim 6. Lines 3-6 preserve all the invariants.

Proof. Because we keep the changes only when the search fails, the invariant 1 is satisfied. The invariant 2 follows from Lemma 14 against $P:=\left(v_{0}, \ldots, v_{i}\right)$. The invariants 3 and 4 are trivial. Note that the current integral path $I$ is still under processing. 
Claim 7. When the repetition of lines 3-6 ends, there exists no minimum half-integral $\mathcal{F}$-cover $x^{\prime}$ satisfying $a(I)<a_{x^{\prime}}(I) \leq b_{x^{\prime}}(I) \leq b(I)$.

Proof. The repetition ends when $a(I)$ becomes equal to $b(I)$ or when a $y$-augmenting path or pair is found. The former case is trivial. In the latter case, let $\mathcal{F}^{\prime}$ be the set of the conflicting walks after contracting $\left(v_{0}, \ldots, v_{a(I)+1}\right)$. Suppose that there exists a minimum half-integral $\mathcal{F}$ cover $x^{\prime}$ satisfying the condition in the lemma. Then, from Lemma $14, x^{\prime}$ is also a half-integral $\mathcal{F}^{\prime}$-cover. In contrast, the size of the maximum half-integral $\mathcal{F}^{\prime}$-packing $y^{\prime}$ is strictly larger than $|y|$ because a $y$-augmenting path or pair is found. Thus, we have $\left|y^{\prime}\right|>|y|=\left|x^{\prime}\right| \geq\left|y^{\prime}\right|$, which is a contradiction.

After repeating lines 3-6, we repeat the following while $a(I)<b(I)$ holds (lines 7-10). Let $i:=b(I)-1$. We first contract the implicational path $\left(v_{\ell}, \ldots, v_{i}\right)$, and then we restart the search by setting $s \leftarrow v_{i}^{\prime}$. If the restarted search fails, we continue the repetition, and if the restarted search succeeds, we rewind all the changes in this step and exit the repetition. By the same argument as in the case of lines 3-6, all the invariants are preserved and, when the repetition ends, there exists no minimum half-integral $\mathcal{F}$-cover $x^{\prime}$ satisfying $a(I) \leq a_{x^{\prime}}(I) \leq b_{x^{\prime}}(I)<b(I)$. Thus, the invariant 3 is satisfied for $I$ when we have finished processing $I$.

Next, we repeat the following for each spoke $S=\left(v_{0}, \ldots, v_{\ell}\right)$ while $a(S)<\ell$ holds (lines 12-15). Let $i:=a(S)+1$. We first contract the implicational path $\left(v_{0}, \ldots, v_{i}\right)$, and then restart the search by setting $s \leftarrow v_{i}^{\prime}$. We continue the repetition if the restarted search fails, and, otherwise, we rewind all the changes in this step and exit the repetition. By the same argument as in the case of integral paths, all the invariants are preserved and, when the repetition ends, there exists no minimum half-integral $\mathcal{F}$-cover $x^{\prime}$ satisfying $a(S)<a_{x^{\prime}}(S)$. Thus, the invariant 4 is satisfied for $S$ when we have finished processing $S$.

Finally, we analyze the running time of Algorithm 2. The number of inserted edges is $O(n)$, and the number of while loops (lines $3-6,7-10$, or 12-15) is $2 k$. For each while loop, a series of the restarted searches can be regarded as a single execution of Algorithm 1, which takes $O(m T)$ time. Therefore, the total running time is $O(k m T)$. Thus, we obtain Theorem 3.

\section{Linear-Time FPT Algorithms}

\subsection{Algorithm for 0/1/all Deletion}

This section proposes an $O\left(d^{2 k} k m\right)$-time algorithm for 0/1/ALL DeLETION. Our algorithm is based on the branch-and-bound algorithm in [19]. We exploit the farthest minimum half-integral $\mathcal{F}$-cover and parallel unit-propagation to obtain a linear-time FPT algorithm.

Let $I=\left(C, \varphi_{A}\right)$ be a pair of $0 / 1 /$ all constraints $C$ on a variable set $V$ and a partial-assignment $\varphi_{A}$ on a subset $A \subseteq V$. We denote $\mathcal{F}_{C, \varphi_{A}}$ by $\mathcal{F}_{I}$. For a variable $u \in V \backslash A$ and an element $a \in D(u)$, we denote by $I[u \leftarrow a]$ a pair $\left(C, \varphi_{A \cup\{u\}}\right)$ such that $\varphi_{A \cup\{u\}}(u)=a$. We denote by $I-u$ a pair $\left(C[V \backslash\{u\}], \varphi_{A \backslash\{u\}}\right)$ for a variable $u \in V$, where $\varphi_{A \backslash\{u\}}$ is the restriction of $\varphi_{A}$ to $A \backslash\{u\}$. We call this operation deleting $u$. Let $N_{u}:=\left\{v \in A \mid u v \in E, \varphi_{A}\right.$ does not satisfy $\left.C_{u v}\right\}$ for a variable $u \in A$. We denote by $I / u$ a pair $\left(C\left[V \backslash\left(\{u\} \cup N_{u}\right)\right], \varphi_{A^{\prime}}\right)$ such that $A^{\prime}=\left(A \cup\left\{v \mid C_{u v}\left(\varphi_{A}(u)\right) \neq\right.\right.$ all $\}) \backslash\left(\{u\} \cup N_{u}\right)$ and $\varphi_{A^{\prime}}(v)=C_{u v}\left(\varphi_{A}(u)\right)$ for $v \in A^{\prime} \backslash A$. We call this operation fixing $u$. We can obtain an incremental-test oracle for $I / u$ by setting $\mathcal{I}(v)=\mathcal{A}(\mathcal{I}(u), u v)$ for each $v \in A^{\prime} \backslash A$. We can observe the following. 
Lemma 17. The following holds for any pair $I=\left(C, \varphi_{A}\right)$ and $u \in V$.

1. I admits a deletion set of size $k$ containing $u$ if and only if $I-u$ admits a deletion set of size $k-1$.

2. For any half-integral $\mathcal{F}_{I-u}$-cover $x^{\prime}$, the following function $x$ is a half-integral $\mathcal{F}_{I^{-} \text {-cover: }}$ $x(u)=1$ and $x(v)=x^{\prime}(v)$ for $v \in V \backslash\{u\}$.

Proof. First, we prove the first claim. For a deletion set $X$ for $I$ containing $u, X \backslash\{u\}$ is a deletion set for $I-u$. For a deletion set $X^{\prime}$ for $I-u, X^{\prime} \cup\{u\}$ is a deletion set for $I$.

Next, we prove the second claim. Let $W$ be a walk in $\mathcal{F}_{I}$. We have $x(V(W)) \geq x^{\prime}(V(W)) \geq 1$ if $W \in \mathcal{F}_{I-u}$. Otherwise, $W$ visits $u$; therefore, we have $x(V(W)) \geq x(u)=1$.

Lemma 18. The following holds for any pair $I=\left(C, \varphi_{A}\right)$ and $u \in A$.

1. I admits a deletion set of size $k$ not containing $u$ if and only if $I / u$ admits a deletion set of size $k-\left|N_{u}\right|$.

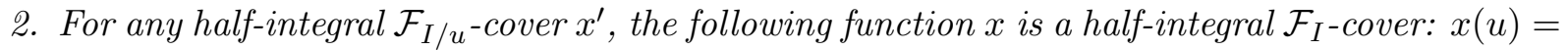
$0, x(v)=1$ for $v \in N_{u}$, and $x(v)=x^{\prime}(v)$ for $v \in V \backslash\left(\{u\} \cup N_{u}\right)$.

Proof. First, we prove the first claim. Let $X$ be a deletion set for $I$ not containing $u$. Because $X$ must contain all of $N_{u}, X \backslash N_{u}$ is a deletion set for $I / u$ of size $|X|-\left|N_{u}\right|$. For a deletion set $X^{\prime}$ for $I / u, X^{\prime} \cup N_{u}$ is a deletion set for $I$.

Next, we prove the second claim. Let $W$ be a walk in $\mathcal{F}_{I}$. If $W \in \mathcal{F}_{I / u}$, we have $x(V(W)) \geq$ $x^{\prime}(V(W)) \geq 1$. If $W$ visits a vertex in $N_{u}$, we have $x(V(W)) \geq 1$. Otherwise, we can write $W=u v \circ W^{\prime}$. Then, we have $W^{\prime} \in \mathcal{F}_{I / u}$, and thus, we have $x(V(W)) \geq x^{\prime}\left(V\left(W^{\prime}\right)\right) \geq 1$.

For a minimum half-integral $\mathcal{F}_{I}$-cover $x$, we define an operation called a persistency reduction as follows. We first delete every vertex in $x^{-1}(1)$ in an arbitrary order, and then fix every vertex in

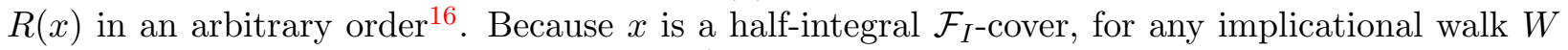
with $x(V(W) \backslash\{t(W)\})=0$ and $x(t(W)) \leq \frac{1}{2}$, the value $\operatorname{imp}(W)$ depends only on $t(W)$. Therefore, the ordering does not affect the result and $N_{u}=\emptyset$ for every fixing. We denote the obtained pair $\left(C\left[V \backslash\left(x^{-1}(1) \cup R(x)\right)\right], \varphi_{A^{\prime}}\right)$ by $I / x$.

Lemma 19. I admits a deletion set of size $k$ if and only if $I / x$ admits a deletion set of size $k-\left|x^{-1}(1)\right|$.

Proof. From Theorem 1, there exists a minimum deletion set $X$ for $I$ such that $x^{-1}(1) \subseteq X \subseteq$ $V \backslash R(x)$. Therefore, from Lemma 17 and 18, the claim holds.

Lemma 20. For a farthest minimum half-integral $\mathcal{F}_{I}$-cover $x$, the restriction of $x$ to $V \backslash\left(x^{-1}(1) \cup\right.$ $R(x))$ is the unique minimum half-integral $\mathcal{F}_{I / x^{-}}$cover.

Proof. Let $x^{\prime}$ be the restriction of $x$. Then, $x^{\prime}$ is a half-integral $\mathcal{F}_{I / x^{-c o v e r}}$ because $x^{\prime}(u)=\frac{1}{2}$ for every $u \in A^{\prime}$. Suppose that there exists a half-integral $\mathcal{F}_{I / x^{-c o v e r}} z^{\prime}$ with $\left|z^{\prime}\right| \leq\left|x^{\prime}\right|$ and $z^{\prime} \neq x^{\prime}$. From Lemmas 17 and 18, the following function $z$ is a half-integral $\mathcal{F}$-cover: $z(u)=z^{\prime}(u)$ for $u \in V \backslash\left(x^{-1}(1) \cup R(x)\right), z(u)=1$ for $u \in x^{-1}(1), z(u)=0$ for $u \in R(x)$. Then, we have $|z|=\left|z^{\prime}\right|+\left|x^{-1}(1)\right| \leq\left|x^{\prime}\right|+\left|x^{-1}(1)\right|=|x|$. Therefore, $z$ is a minimum half-integral $\mathcal{F}$-cover dominating $x$, which is a contradiction.

\footnotetext{
${ }^{16}$ We pick an arbitrary vertex $u \in R(x) \cap A$ and fix $u$. This changes $R(x)$ and $A$, and we repeat the process until $R(x)$ becomes the empty set.
} 


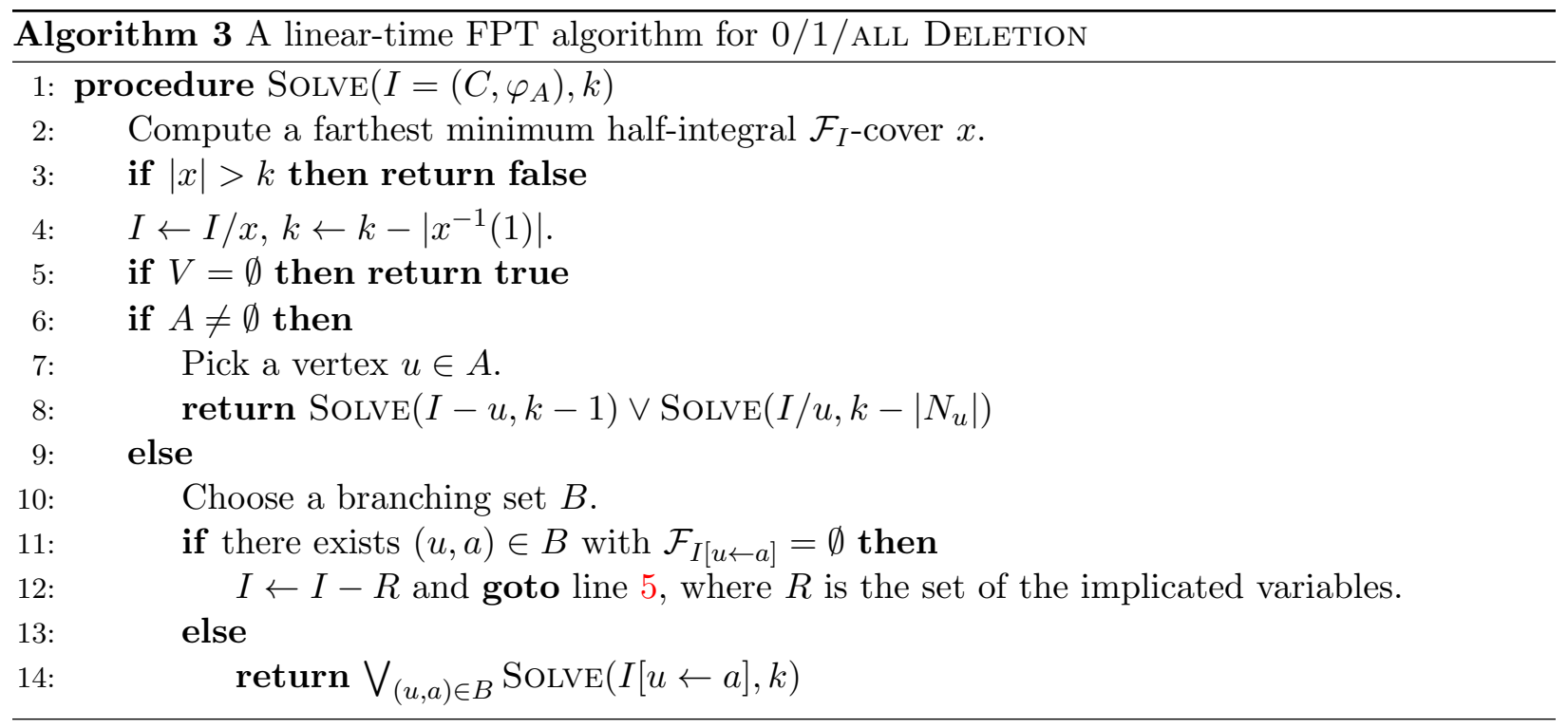

Let $I=\left(C, \varphi_{\emptyset}\right)$ be a pair with $A=\emptyset$. A set of pairs $B \subseteq\{(u, a) \mid u \in V, a \in D(u)\}$ is called a branching set for $I$ if it has the following property: any deletion set for $I$ is a deletion set for at least one of $I[u \leftarrow a]$ with $(u, a) \in B$. Note that any deletion set for $I[u \leftarrow a]$ is a deletion set for $I$. The running time of our algorithm depends on the choice of branching sets. In general, we can use the following standard choice: pick a vertex $u \in V$ and set $B:=\{(u, a) \mid a \in D(u)\}$. In the next section, we choose different branching sets for problem-specific improvements.

Lemma 21. $B:=\{(u, a) \mid a \in D(u)\}$ for some $u \in V$ is a branching set.

Proof. Let $X$ be a deletion set for $I$, and let $\varphi$ be a satisfying assignment for $C[V \backslash X]$. If $u \in X, X$ is a deletion set for every $I[u \leftarrow a]$. Otherwise, $X$ is a deletion set for $I[u \leftarrow a]$ with $a=\varphi(u)$.

We now provide a linear-time FPT algorithm for 0/1/ALL Deletion (Algorithm 3). We denote

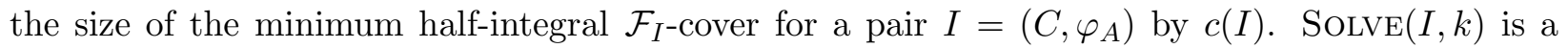
procedure that returns true if and only if $I$ admits a deletion set of size at most $k$. We prove the following.

Theorem 4. Let $I=\left(C, \varphi_{A}\right)$ be a pair of a set $C$ of $0 / 1 /$ all constraints on a variable set $V$ and a partial assignment $\varphi_{A}$ for a subset $A \subseteq V$. We are given the primal graph of $C$, the set $A$, an $O(T)$-time incremental-test oracle for $\left(C, \varphi_{A}\right)$, and an integer $k$. Under the following assumptions, Algorithm 3 correctly answers whether $\left(C, \varphi_{A}\right)$ admits a deletion set of size at most $k$ or not in $O\left(\max (2, b)^{2(k-c(I))} k m T\right)$ time, where $b$ is the integer in the assumption and $m$ is the number of constraints.

1. For any $V^{\prime} \subseteq V$, we can choose a branching set for $\left(C\left[V^{\prime}\right], \varphi_{\emptyset}\right)$ of size at most $b$.

2. For any $V^{\prime} \subseteq V$ and any $(u, a) \in B$ for a possible branching set $B$ for $\left(C\left[V^{\prime}\right], \varphi_{\emptyset}\right)$, we have an $O(T)$-time incremental-test oracle for $\left(C\left[V^{\prime}\right], \varphi_{\{u\}}\right)$ with $\varphi_{\{u\}}(u)=a$.

Note that for 0/1/ALL DELETION, we assume that each constraint is given as a table of size $O(d)$. Therefore, the naive implementation of the incremental-test oracle runs in a constant time. When 
using the naive implementation of the incremental-test oracle, the second assumption trivially holds. Thus, by using the standard choice of branching sets, the algorithm runs in $O\left(d^{2(k-c(I))} k m\right)=$ $O\left(d^{2 k} k m\right)$ time.

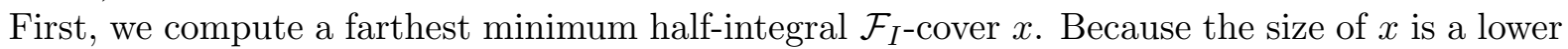
bound on the size of the minimum deletion set, if $|x|>k$ holds, there exists no deletion set of size at most $k$. Otherwise, we have $k-c(I) \geq 0$. From Lemma 19, we can apply the persistency reduction and decrease $k$ by $\left|x^{-1}(1)\right|$. This does not change the difference $k-c(I)$ because $c(I)$ also decreases by $\left|x^{-1}(1)\right|$. We rename the reduced instance to $I=\left(C, \varphi_{A}\right)$ for simplicity of the notation, and let $G=(V, E)$ be the primal graph of $C$. We rename the restriction of $x$ to $V$ to $x$.

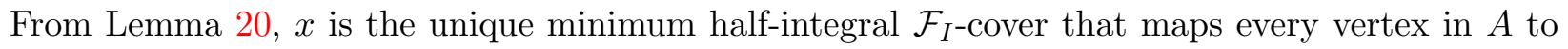
$\frac{1}{2}$ and every other vertex to 0 . This part can be done in $O(k m T)$ time and we can construct an incremental-test oracle for the new $I$ from the incremental-test oracle for the old $I$.

If $A \neq \emptyset$, we pick a vertex $u \in A$ and $\operatorname{return} \operatorname{Solve}(I-u, k-1) \vee \operatorname{Solve}\left(I / u, k-\left|N_{u}\right|\right)$.

Claim 8. I admits a deletion set of size at most $k$ if and only if $I-u$ admits a deletion set of size at most $k-1$ or $I / u$ admits a deletion set of size at most $k-\left|N_{u}\right|$. Moreover, both of $k-1-c(I-u)<k-c(I)$ and $k-\left|N_{u}\right|-c(I / u)<k-c(I)$ hold.

Proof. The first claim follows from Lemma 17 and 18. We now prove the second claim.

Suppose that $I-u$ admits a half-integral $\mathcal{F}_{I-u}$-cover $z^{\prime}$ of size at most $|x|-1=c(I)-1$. Then,

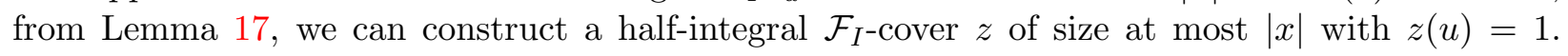

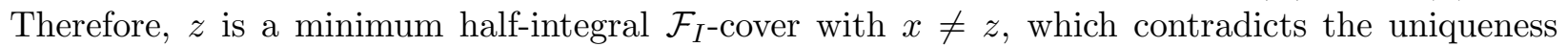
of $x$.

Suppose that $I / u$ admits a half-integral $\mathcal{F}_{I / u}$-cover $z^{\prime}$ of size at most $|x|-\left|N_{u}\right|=c(I)-\left|N_{u}\right|$. Then, from Lemma 18 , we can construct a half-integral $\mathcal{F}_{I}$-cover $z$ of size at most $|x|$ with $z(u)=0$.

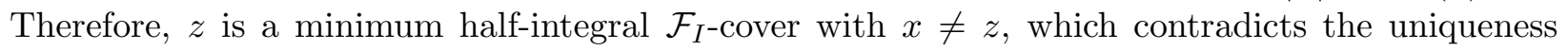
of $x$.

If $A=\emptyset$, we choose a branching set $B$ of size at most $b$. For each $(u, a) \in B$, we check whether $\mathcal{F}_{I[u \leftarrow a]}=\emptyset$ or not by the unit-propagation (i.e., by applying Algorithm 1 against the empty packing). If there exists such $(u, a)$, let $R$ be the set of the implicated variables (i.e., $R(\mathbf{0})$ for the empty cover $\mathbf{0}$ that always returns 0$)$. Let $m^{\prime}$ be the number of edges incident to a vertex in $R$, and suppose that $(u, a)$ is the pair minimizing $m^{\prime}$. The unit-propagation for $(u, a)$ takes only $O\left(m^{\prime} T\right)$ time. Hence, by running the unit-propagation for every $(u, a) \in B$ in parallel, which is simulated in a single processor in a round-robin fashion, we can find such $(u, a)$ in $O\left(b m^{\prime} T\right)$ time. We then set $I \leftarrow I-R$, where $I-R$ is the pair obtained by deleting every vertex $v \in R$, and go back to line 5 .

Claim 9. I admits a deletion set of size at most $k$ if and only if $I-R$ admits a deletion set of size at most $k$.

Proof. Any deletion set for $I$ is also a deletion set for $I-R$. Let $X$ be a deletion set for $I-R$, and let $\varphi_{V \backslash(R \cup X)}$ be a satisfying assignment for $C[V \backslash(R \cup X)]$. From the construction of $R$, $C[R]$ admits a satisfying assignment $\varphi_{R}$, and every constraint $C_{v w}$ with $v \in R$ and $w \notin R$ is a two-fan $(\varphi(v)=a) \vee(\varphi(w)=b)$ with $a=\varphi_{R}(v)$. Therefore, an assignment $\varphi_{V \backslash X}$ such that $\varphi_{V \backslash X}(v)=\varphi_{R}(v)$ for $v \in R$ and $\varphi_{V \backslash X}(v)=\varphi_{V \backslash(R \cup X)}(v)$ for $v \in V \backslash(R \cup X)$ satisfies $C[V \backslash X]$. Thus, $X$ is also a deletion set for $I$. 
If $\mathcal{F}_{I[u \leftarrow a]} \neq \emptyset$ for all $(u, a) \in B$, we return $\bigvee_{(u, a) \in B} \operatorname{SoLvE}(I[u \leftarrow a])$.

Claim 10. I admits a deletion set of size at most $k$ if and only if at least one of $I[u \leftarrow a]$ admits a deletion set of size $k$. Moreover, $k-c(I[u \leftarrow a])<k-c(I)$ for every $(u, a) \in B$.

Proof. The first claim follows from the definition of the branching set. Because $\mathcal{F}_{I[u \leftarrow a]} \neq \emptyset$, we have $k-c(I[u \leftarrow a]) \leq k-\frac{1}{2}<k=k-c(I)$.

We now have proved the correctness of the algorithm. Finally, we analyze the running time. Let $\mathcal{T}(\Delta)$ be the running time of $\operatorname{Solve}(I, k)$ when $\Delta:=k-c(I)$. We can compute a farthest minimum half-integral $\mathcal{F}_{I}$-cover in $O(k m T)$ time. If $A \neq \emptyset$, we branch into two cases and $\Delta$ decreases by at least $\frac{1}{2}$ for each case. Therefore, we have $\mathcal{T}(\Delta) \leq 2 \mathcal{T}\left(\Delta-\frac{1}{2}\right)+O(k m T)$. If $A=\emptyset$, we search for $(u, a) \in B$ with $\mathcal{F}_{I[u \leftarrow a]}=\emptyset$ by the parallel unit-propagation. If there exists such $(u, a)$, the parallel unit-propagation takes $O\left(b m^{\prime} T\right)$ time, and $m$ decreases by $m^{\prime}$. Therefore, in $O(b m T)$ time, we reach to the state that either $V=\emptyset$ or there exists no such $(u, a)$. In the latter

case, we branch into at most $b$ cases, and $\Delta$ decreases by at least $\frac{1}{2}$ for each case. Therefore, we have $\mathcal{T}(\Delta) \leq b \mathcal{T}\left(\Delta-\frac{1}{2}\right)+O(k m T+b m T)$. Thus, we have $\mathcal{T}(\Delta)=O\left(\max (2, b)^{2 \Delta} k m T\right)$.

\subsection{Applications to Other Problems}

Finally, we show that various NP-hard problems can be expressed as a special case of $0 / 1 /$ ALL Deletion. Note that we use $A=\emptyset$ for every problem other than Node Multiway Cut. We obtain a linear-time FPT algorithm for each problem by giving an incremental-test oracle and a specialized choice of branching sets.

Node Unique Label Cover Parameter: $k,|\Sigma|$

Input: A finite alphabet $\Sigma$, a graph $G=(V, E)$, a permutation $\pi_{e}$ of $\Sigma$ for every edge $e \in \hat{E}$ such that $\pi_{u v}=\pi_{v u}^{-1}$ given as a table of size $|\Sigma|$, and an integer $k$.

Question: Is there a pair of set $X \subseteq V$ of at most $k$ vertices and assignment $\varphi: V \backslash X \rightarrow \Sigma$ such that $\pi_{u v}(\varphi(u))=\varphi(v)$ for every $u v \in E[V \backslash X]$ ?

TWO-FAN DELETION

Parameter: $k$

Input: A set of variables $V$, a set of two-fan constraints on $V$ of the form $(\varphi(u)=a) \vee(\varphi(v)=$ $b)$ given as a pair $(a, b)$, and an integer $k$.

Question: Is there a pair of set $X \subseteq V$ of at most $k$ variables and assignment $\varphi$ satisfying every constraint $C_{u v} \in C[V \backslash X]$ ?

These two problems are special cases of $0 / 1$ /ALL DELETION such that the set of constraints is limited to permutation or two-fan constraints. Note that the size of the domain is not a parameter for Two-fan Deletion. The naive implementation of the incremental-test oracle for these problems runs in a constant time. Thus, we can solve Node Unique Label Cover in $O\left(|\Sigma|^{2 k} k m\right)$ time. We can use the following choice of a branching set for TWO-FAN DELETION: pick a two-fan constraint $(\varphi(u)=a) \vee(\varphi(v)=b)$ and set $B:=\{(u, a),(v, b)\}$. Thus, we can solve TwO-FAN DELETION in $O\left(2^{2 k} k m\right)=O\left(4^{k} k m\right)$ time.

Lemma 22. $B:=\{(u, a),(v, b)\}$ for a two-fan constraint $(\varphi(u)=a) \vee(\varphi(v)=b)$ is a branching set. 
Proof. Let $X$ be a deletion set for $I$ and let $\varphi$ be an assignment for $V \backslash X$ satisfying $C[V \backslash X]$. If $u \in X, X$ is a deletion set for $I[u \leftarrow a]$, and if $v \in X, X$ is a deletion set for $I[v \leftarrow b]$. Otherwise, at least one of $\varphi(u)=a$ or $\varphi(v)=b$ holds. $X$ is a deletion set for $I[u \leftarrow a]$ in the former case, while $X$ is a deletion set for $I[v \leftarrow b]$ in the latter case.

The next two problems generalize Pseudoforest Deletion [5] in different directions, where a pseudoforest is a graph in which the number of edges is at most the number of vertices for every connected component.

A graph is called monochromatically orientable if there exists an edge orientation such that, for every vertex, all the incoming edges are monochromatic. It is known that a graph is a pseudoforest if and only if there exists an edge orientation such that, for every vertex, the number of incoming edges is at most one. Therefore, when every edge has a distinct color, a graph is monochromatically orientable if and only if it is a pseudoforest. Thus, the following problem is a generalization of PSEudoforest Deletion.

Monochromatically Orientable Deletion

Parameter: $k$

Input: An edge-colored graph $G=(V, E)$ and an integer $k$.

Question: Is there a set $X \subseteq V$ of at most $k$ vertices such that $G-X$ is monochromatically orientable?

We can solve Monochromatically Orientable Deletion in $O\left(4^{k} \mathrm{~km}\right)$ time by the following reduction to Two-fan Deletion. Let $L$ be the set of colors. For each vertex $v \in V$, we create a variable $v$ with domain $D(v)=L$, which represents the color of the incoming edges. We create a two-fan constraint $(\varphi(u)=c) \vee(\varphi(v)=c)$ for each edge $u v \in E$ of color $c \in L$.

Lemma 23. A graph $G=(V, E)$ is monochromatically orientable if and only if the corresponding set $C$ of two-fan constraints is satisfiable.

Proof. From a monochromatic orientation of $G$, we can construct a satisfying assignment $\varphi$ as follows: we set $\varphi(v):=c$ for each vertex $v \in V$, where $c$ is the unique color of the incoming edges or an arbitrary color if $v$ has no incoming edges. From a satisfying assignment $\varphi$, we can construct a monochromatic orientation by orienting each edge $u v \in E$ of color $c$ so that the edge is directed toward $u$ if $\varphi(u)=c$, and toward $v$ otherwise.

Another generalization of PSEUdoforest DELetion is presented as follows. For a graph $G=(V, E)$ and an edge $e=u v \in E$, contracting $e$ is an operation deleting the edge $e$ and merging $u$ and $v$ into a new vertex $e$. Note that this operation may create parallel edges (edges $u w$ and $v w$ become parallel edges) and self-loops. If $G$ has parallel edges connecting $u$ and $v$, the operation only removes one of them, and the rest becomes self-loops.

Let $S$ be a subset of edges. A subset $X \subseteq V$ is called a subset feedback vertex set if $G-X$ has no simple cycle passing through an edge of $S$, or equivalently, the graph obtained from $G-X$ by contracting every edge $e \in E[V \backslash X] \backslash S$ is a forest. Similarly, we call $X$ a subset pseudoforest deletion set if the graph obtained from $G-X$ by contracting every edge $e \in E[V \backslash X] \backslash S$ is a pseudoforest.

Subset Pseudoforest Deletion

Parameter: $k$

Input: A graph $G=(V, E)$, a set $S \subseteq E$, and an integer $k$.

Question: Is there a set $X \subseteq V$ of at most $k$ vertices such that the graph obtained from $G-X$ by contracting every edge $e \in E[V \backslash X] \backslash S$ is a pseudoforest? 
This problem can be expressed as 0/1/ALL DeLETion as follows. Every vertex $v$ has the same domain $D(v)=S$. We introduce a two-fan constraint $(\varphi(u)=e) \vee(\varphi(v)=e)$ for every edge $e=u v \in S$. We also introduce an equality (identity permutation) constraint $\varphi(u)=\varphi(v)$ for every edge $u v \notin S$. Let $C$ be the obtained set of constraints. If $C\left[V^{\prime}\right]$ has no two-fan constraints for some $V^{\prime} \subseteq V,\left(C\left[V^{\prime}\right], \varphi_{\emptyset}\right)$ has a deletion set of size zero. Therefore, we can use the same choice of branching sets as for Two-fAn Deletion. Thus, the algorithm runs in $O\left(4^{k} k m\right)$ time. The correctness of the expression follows from the following lemma.

Lemma 24. Let $G=(V, E)$ be a graph with a subset $S \subseteq E$ and let $C$ be the corresponding set of $0 / 1 /$ all constraints. Then, the graph obtained by contracting every edge $e \in E \backslash S$ is a pseudoforest if and only if $C$ is satisfiable.

Proof. We modify $C$ for each contraction of an edge $e=u v$ by removing the constraint $C_{u v}$ and replacing every occurrence of $u$ and $v$ by $e$. When we obtain the graph $G^{\prime}$ by contracting every edge $e \in E \backslash S$, we also obtain the set $C^{\prime}$ of constraints corresponding to $G^{\prime}$. Because every contracted edge $e=u v$ has the equality constraint, $C$ is satisfiable if and only if $C^{\prime}$ is satisfiable. From Lemma 23, $C^{\prime}$ is satisfiable if and only if $G^{\prime}$ is a pseudoforest. Therefore, $C$ is satisfiable if and only if $G^{\prime}$ is a pseudoforest.

As mentioned in Section 1.3, Node Multiway Cut is also a special case of our problem.

Node Multiway Cut

Parameter: $k$

Input: A graph $G=(V, E)$, a set of terminals $T \subseteq V$, and an integer $k$.

Question: Is there a set $X \subseteq V \backslash T$ of size at most $k$ such that every terminal in $T$ lies in a different connected component of $G-X$ ?

This problem can be expressed as 0/1/ALL DeLETion as follows. First, we split each terminal $s \in T$ as follows to make $s$ undeletable: for each edge $s v \in \delta(s)$, we create a new vertex $s_{v}$ and replace the edge $s v$ with $s_{v} v$. Let $G^{\prime}=\left(V^{\prime}, E^{\prime}\right)$ be the obtained graph. We introduce a variable $v$ with $D(v):=T$ for each vertex $v \in V^{\prime}$ and an equality (identity permutation) constraint $\varphi(u)=\varphi(v)$ for each edge $u v \in E^{\prime}$. Finally, we set $A=\left\{s_{v} \mid s \in T, s v \in \delta(s)\right\}$ and $\varphi_{A}\left(s_{v}\right):=s$. We can observe that a minimum deletion set $X$ avoiding every $s_{v}$ always exists because each vertex $s_{v}$ has degree one, and such $X$ is actually a minimum multiway cut.

Let $I=\left(C, \varphi_{A}\right)$ be the obtained instance. We can construct a multiway cut of size at most $2|x|$

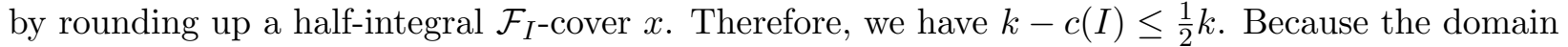
size is $|T|=O(n)$, the naive implementation of the incremental-test oracle runs in a constant time. Because any $I=\left(C\left[V^{\prime}\right], \varphi_{\emptyset}\right)$ has a deletion set of size zero, we do not need branching sets. Thus, we can solve Node Multiway Cut in $O\left(2^{2 \cdot \frac{1}{2} k} k m\right)=O\left(2^{k} k m\right)$ time.

We finally show Group Feedback Vertex Set and its applications.

Group Feedback Vertex Set

Parameter: $k$

Input: A group $\Gamma=(D, \cdot)$ given as an $O\left(T_{\Gamma}\right)$-time oracle performing the group operation $(\cdot)$, a $\Gamma$-labeled graph $G=(V, E)$ with labeling $\lambda: \hat{E} \rightarrow D$ with $\lambda(u v) \cdot \lambda(v u)=1_{\Gamma}$ for every $u v \in \hat{E}$, where $1_{\Gamma}$ is the unity of $\Gamma$, and an integer $k$.

Question: Is there a set $X \subseteq V$ of at most $k$ vertices such that $G-X$ has a consistent labeling? That is, is there a labeling $\varphi: V \backslash X \rightarrow D$ such that $\varphi(u) \cdot \lambda(u v)=\varphi(v)$ for every $u v \in E[V \backslash X]$ ? 
This problem can be expressed as 0/1/ALL Deletion because a function $\pi_{e}(a):=a \cdot \lambda(e)$ is a permutation. Note that $G-X$ has a consistent labeling if and only if it admits no non-zero cycles (i.e., it admits no cycle $\left(v_{0}, \ldots, v_{\ell}\right)$ with $\left.\lambda\left(v_{0} v_{1}\right) \cdot \lambda\left(v_{1} v_{2}\right) \cdots \lambda\left(v_{\ell-1} v_{\ell}\right) \neq 1_{\Gamma}\right)$. In contrast to NoDE UNIQUe LABEL COVER, the domain size is not a parameter, and each permutation is given not as a table of size $|D|$, but as an $O\left(T_{\Gamma}\right)$-time oracle answering $a \cdot b$ for given $a, b \in D$. Therefore, the naive implementation of the incremental-test oracle runs in $O\left(T_{\Gamma}\right)$ time. We can use the following choice of a branching set: pick a vertex $s$ and set $B:=\left\{\left(s, 1_{\Gamma}\right)\right\}$. Thus, we can solve Group FEEDBACK Vertex Set in $O\left(2^{2 k} k m\right)=O\left(4^{k} k m\right)$ time.

Lemma 25. $B:=\left\{\left(s, 1_{\Gamma}\right)\right\}$ is a branching set for Group Feedback Vertex Set.

Proof. Let $X$ be a deletion set for $I$, and let $\varphi$ be an assignment for $V \backslash X$ satisfying $C[V \backslash X]$. If $s \in X, X$ is a deletion set for $I\left[s \leftarrow 1_{\Gamma}\right]$; otherwise, $\varphi^{\prime}$ such that $\varphi^{\prime}(v):=\varphi(v) \cdot \varphi(s)^{-1}$ is a satisfying assignment for $C[V \backslash X]$ with $\varphi^{\prime}(s)=1_{\Gamma}$.

Subset Feedback Vertex Set

Input: A graph $G=(V, E)$, a set $S \subseteq E$, and an integer $k$.

Question: Is there a set $X \subseteq V$ of at most $k$ vertices such that no cycle passes through an edge of $S$ in $G-X$ ?

This problem can be expressed as Group Feedback Vertex Set as follows. We use a group $\Gamma=\left(2^{S}, \oplus\right)$, where $\oplus$ is the XOR operator $(X \oplus Y=(X \backslash Y) \cup(Y \backslash X))$. We set $\lambda(e)=\{e\}$ for each edge $e \in S$ and $\lambda(e)=\emptyset$ for each edge $e \in E \backslash S$. Then, a cycle is non-zero if and only if it contains an edge in $S$.

The group operation takes $O(|S|)=O(m)$ time. Therefore, the naive implementation of the incremental-test oracle takes $O(m)$ time. We now provide a constant-time incremental-test oracle for $\left(C\left[V^{\prime}\right], \varphi_{\{s\}}\right)$ with $\varphi_{\{s\}}(s)=\emptyset$. We use the following implementation.

$$
U:=S \cup\{\epsilon\} . \quad \mathcal{I}(s)=\epsilon . \quad \mathcal{A}(a, e)=\left\{\begin{array}{ll}
e & (e \in S) \\
a & (e \notin S) .
\end{array} \quad \mathcal{T}(a, b)= \begin{cases}\text { true } & (a \neq b) \\
\text { false } & (a=b) .\end{cases}\right.
$$

For a walk $W$, the function $\mathcal{A}^{*}(W)$ returns the last edge of $W$ contained in $S$ or $\epsilon$ if $W$ contains no edges in $S$. Then, for a single-branching pair $(P, Q)$, we have $P \not \equiv Q \Longleftrightarrow$ the simple cycle contained in $P \circ Q^{-1}$ contains an edge in $S \Longleftrightarrow \mathcal{A}^{*}(P) \neq \mathcal{A}^{*}(Q)$. Therefore, the abovementioned implementation is correct. Thus, we can solve Subset Feedback Vertex Set in $O\left(4^{k} k m\right)$ time.

Non-monochromatic Cycle Transversal

Parameter: $k$

Input: An edge-colored graph $G=(V, E)$ and an integer $k$.

Question: Is there a set $X \subseteq V$ of at most $k$ vertices such that $G-X$ contains no nonmonochromatic cycles?

This problem can be expressed as Group Feedback Vertex Set as follows. Let $L$ be the set of colors, and let $c(e) \in L$ denote the color of an edge $e$. We use the group $\Gamma=\left(2^{V \times L}, \oplus\right)$. We set $\lambda(e)=\{(u, c(e)),(v, c(e))\}$ for each edge $e=u v \in E$. Then, a cycle is non-zero if and only if it is non-monochromatic.

The naive implementation of the incremental-test oracle takes $O(n|L|)=O(n m)$ time. We now provide a constant-time incremental-test oracle for $\left(C\left[V^{\prime}\right], \varphi_{\{s\}}\right)$ with $\varphi_{A}(s)=\emptyset$. We use the 
following implementation.

$$
\begin{gathered}
U:=(V \times L) \cup\{\epsilon, *\} . \quad \mathcal{I}(s)=\epsilon . \quad \mathcal{T}(a, b)= \begin{cases}\text { true } & (* \neq a \neq b \neq *) \\
\text { false } & \text { otherwise. }\end{cases} \\
\mathcal{A}(\epsilon, s v)=(s, c(s v)) . \quad \mathcal{A}((w, c), u v)= \begin{cases}* & (w=v \wedge c=c(u v)) \\
(w, c) & (w \neq v \wedge c=c(u v)) \\
(u, c(u v)) & (c \neq c(u v)) .\end{cases}
\end{gathered}
$$

Let $W=\left(v_{0}, \ldots, v_{\ell}\right)$ be a walk with $\ell>0$, and let $c=c\left(v_{\ell-1} v_{\ell}\right)$. A suffix $\left(v_{i}, \ldots, v_{\ell}\right)$ is called the longest monochromatic suffix of $W$ if $c\left(v_{j} v_{j+1}\right)=c$ for every $j \geq i$ and $c\left(v_{i-1} v_{i}\right) \neq c$ or $i=0$ holds. We can observe that the longest monochromatic suffix of $W$ starts from $v_{i}$ and has the color $c$ if $\mathcal{A}^{*}(W)=\left(v_{i}, c\right)$, and the longest monochromatic suffix of $W$ forms a monochromatic cycle if $\mathcal{A}^{*}(W)=*$. Then, for a single-branching pair $(P, Q)$, we have $P \not \equiv Q \Longleftrightarrow$ the simple cycle contained in $P \circ Q^{-1}$ is non-monochromatic $\Longleftrightarrow$ none of $P$ and $Q$ induces a monochromatic cycle and the longest monochromatic suffixes of $P$ and $Q$ start from different vertices or have different colors $\Longleftrightarrow * \neq \mathcal{A}^{*}(P) \neq \mathcal{A}^{*}(Q) \neq *$. Therefore, the abovementioned implementation is correct. Thus, we can solve Non-monochromatic Cycle Transversal in $O\left(4^{k} k m\right)$ time.

\section{References}

[1] M. A. Babenko. A fast algorithm for the path 2-packing problem. Theory of Computing Systems, 46(1):59-79, 2010.

[2] M. L. Balinski. Integer programming: Methods, uses, computations. Management Science, 12(3):253-313, 1965.

[3] A. Becker, R. Bar-Yehuda, and D. Geiger. Randomized algorithms for the loop cutset problem. Journal of Artificial Intelligence Research, 12:219-234, 2000.

[4] H. L. Bodlaender. A linear-time algorithm for finding tree-decompositions of small treewidth. SIAM Journal on Computing, 25(6):1305-1317, 2006.

[5] H. L. Bodlaender, H. Ono, and Y. Otachi. A faster parameterized algorithm for pseudoforest deletion. In Proceedings of the 11th International Symposium on Parameterized and Exact Computation (IPEC 2016), pages 7:1-7:12, 2017.

[6] J. Chen, Y. Liu, and S. Lu. An improved parameterized algorithm for the minimum node multiway cut problem. Algorithmica, 55(1):1-13, 2009.

[7] M. C. Cooper, D. A. Cohen, and P. G. Jeavons. Characterising tractable constraints. Artificial Intelligence, 65(2):347-361, 1994.

[8] M. Cygan, F. V. Fomin, Ł. Kowalik, D. Lokshtanov, D. Marx, M. Pilipczuk, M. Pilipczuk, and S. Saurabh. Parameterized Algorithms. Springer International Publishing, 2015.

[9] M. Cygan, M. Pilipczuk, M. Pilipczuk, and J. O. Wojtaszczyk. On multiway cut parameterized above lower bounds. ACM Transactions on Computation Theory, 5(1):3-11, 2013. 
[10] R. G. Downey and M. R. Fellows. Parameterized Complexity. Springer Verlag, 2012.

[11] J. Edmonds. Paths, trees, and flowers. Canadian Journal of mathematics, pages 449-467, 1965 .

[12] A. Frank and É. Tardos. An application of simultaneous diophantine approximation in combinatorial optimization. Combinatorica, 7(1):49-65, 1987.

[13] S. Fujishige and X. Zhang. New algorithms for the intersection problem of submodular systems. Japan Journal of Industrial and Applied Mathematics, 9(3):369-382, 1992.

[14] N. Garg, V. V. Vazirani, and M. Yannakakis. Multiway cuts in node weighted graphs. Journal of Algorithms, 50(1):49-61, 2004.

[15] S. Guillemot. FPT algorithms for path-transversal and cycle-transversal problems. Discrete Optimization, 8(1):61-71, 2011.

[16] H. Hirai. A dual descent algorithm for node-capacitated multiflow problems and its applications. 2015. arXiv:1508.07065.

[17] Y. Iwata. Linear-time kernelization for feedback vertex set. In Proceedings of 44 th International Colloquium on Automata, Languages, and Programming (ICALP), pages 68:1-68:14, 2017.

[18] Y. Iwata, K. Oka, and Y. Yoshida. Linear-time FPT algorithms via network flow. In Proceedings of the 25th Annual ACM-SIAM Symposium on Discrete Algorithms (SODA), pages 1749-1761, 2014.

[19] Y. Iwata, M. Wahlström, and Y. Yoshida. Half-integrality, LP-branching, and FPT algorithms. SIAM Journal on Computing, 45(4):1377-1411, 2016.

[20] D. Lokshtanov, N. S. Narayanaswamy, V. Raman, M. S. Ramanujan, and S. Saurabh. Faster parameterized algorithms using linear programming. ACM Transactions on Algorithms, 11(2):15:1-15:31, 2014.

[21] D. Lokshtanov, M. S. Ramanujan, and S. Saurabh. Linear time parameterized algorithms for subset feedback vertex set. In Proceedings of 42nd International Colloquium on the Automata, Languages, and Programming (ICALP), pages 935-946, 2015.

[22] D. Lokshtanov, M. S. Ramanujan, and S. Saurabh. A Linear Time Parameterized Algorithm for Directed Feedback Vertex Set. 2016. arXiv:1609.04347.

[23] D. Lokshtanov, M. S. Ramanujan, and S. Saurabh. A linear-time parameterized algorithm for node unique label cover. In Proceedings of the 25th Annual European Symposium on Algorithms (ESA), pages 57:1-57:15, 2017.

[24] G. Nemhauser and L. Trotter. Vertex packing: structural properties and algorithms. Mathematical Programming, 8:232-248, 1975.

[25] G. Pap. A constructive approach to matching and its generalizations. PhD thesis, Eötvös Loránd University, 2006. 
[26] G. Pap. Packing non-returning A-paths. Combinatorica, 27(2):247-251, 2007.

[27] G. Pap. Some new results on node-capacitated packing of $A$-paths. In Proceedings of the 39th Annual ACM Symposium on Theory of Computing (STOC), pages 599-604, 2007.

[28] G. Pap. Packing non-returning A-paths algorithmically. Discrete Mathematics, 308(8):14721488, 2008.

[29] G. Pap. Strongly polynomial time solvability of integral and half-integral node-capacitated multiflow problems. Technical report, EGRES Technical Report, TR-2008-12, Eötvös Loránd University, 2008.

[30] J.-C. Picard and M. Queyranne. On the structure of all minimum cuts in a network and applications. In Combinatorial Optimization II, volume 13 of Mathematical Programming Studies, pages 8-16. Springer Berlin Heidelberg, 1980.

[31] M. S. Ramanujan and S. Saurabh. Linear time parameterized algorithms via skew-symmetric multicuts. In Proceedings of the 25th Annual ACM-SIAM Symposium on Discrete Algorithms (SODA), pages 1739-1748, 2014.

[32] A. Schrijver. Combinatorial optimization: polyhedra and efficiency, volume 24. Springer Science \& Business Media, 2002.

[33] M. Wahlström. LP-branching algorithms based on biased graphs. In Proceedings of the 28th Annual ACM-SIAM Symposium on Discrete Algorithms (SODA), pages 1559-1570, 2017.

[34] Y. Yamaguchi. Packing $A$-paths in group-labelled graphs via linear matroid parity. SIAM Journal on Discrete Mathematics, 30(1):474-492, 2016. 


\section{A Proof of Persistency}

First, we review the results of [19]. Let $D$ be a set containing a special element $\perp$, and let $D_{I}:=D \backslash\{\perp\}$. Consider a poset on $D$ such that $\perp<a$ for every $a \in D_{I}$ and all the other pairs $(a, b)$ with $a \neq b$ are not comparable. Let $a \sqcap b$ be a binary operator that returns the minimum of $a$ and $b$ if they are comparable and returns $\perp$ otherwise. Similarly, let $a \sqcup b$ be a binary operator that returns the maximum of $a$ and $b$ if they are comparable and returns $\perp$ otherwise. We define $\boldsymbol{a} \cdot \boldsymbol{b}:=\left(a_{1} \cdot b_{1}, \ldots, a_{n} \cdot b_{n}\right)$ for a binary operator $(\cdot)$ and tuples of elements $\boldsymbol{a}=\left(a_{1}, \ldots, a_{n}\right)$, $\boldsymbol{b}=\left(b_{1}, \ldots, b_{n}\right) \in D^{n}$. Each index $i$ herein might have a distinct domain $D_{i}$ containing $\perp$, and $D^{n}$ is an abbreviation for $D_{1} \times D_{2} \times \ldots \times D_{n}$. A function $f: D^{n} \rightarrow \mathbb{R}$ is called $k$-submodular if the following inequality holds for any pair of inputs $\boldsymbol{a}, \boldsymbol{b} \in D^{n}$ :

$$
f(\boldsymbol{a})+f(\boldsymbol{b}) \geq f(\boldsymbol{a} \sqcap \boldsymbol{b})+f(\boldsymbol{a} \sqcup \boldsymbol{b}) .
$$

We denote the restriction of a function $f: D^{n} \rightarrow \mathbb{R}$ to the domain $D_{I}^{n}$ by $f_{I}$.

Lemma 26 ( [19]). For a k-submodular function $f: D^{n} \rightarrow \mathbb{R}$ and a minimizer $\boldsymbol{b} \in D^{n}$ of $f$, there exists a minimizer $\boldsymbol{a} \in D_{I}^{n}$ of $f_{I}$ such that $a_{i}=b_{i}$ for every $i$ with $b_{i} \neq \perp$.

Lemma 27 ( [19]). The following three functions are k-submodular.

- For a permutation $\pi$ on $D_{I}$, a function $p_{\pi}: D \times D \rightarrow\{0, \infty\}$ defined as

$$
p_{\pi}(x, y)= \begin{cases}0 & \text { if }(x=y=\perp) \vee\left(x, y \in D_{I} \wedge \pi(x)=y\right) \\ \infty & \text { otherwise }\end{cases}
$$

- For elements $a, b \in D_{I}$, a function $t_{a, b}: D \times D \rightarrow\{0, \infty\}$ defined as

$$
t_{a, b}(x, y)= \begin{cases}0 & \text { if }(x=y=\perp) \vee(x=a) \vee(y=b), \\ \infty & \text { otherwise }\end{cases}
$$

- A function e : $D^{r} \rightarrow\left\{0, \frac{1}{2}, 1\right\}$ defined as

$$
e(\boldsymbol{x})= \begin{cases}0 & \text { if } x_{1}=x_{2}=\cdots=x_{r} \\ 1 & \text { if } \exists i, j \text { such that } \perp \neq x_{i} \neq x_{j} \neq \perp, \\ \frac{1}{2} & \text { otherwise. }\end{cases}
$$

Proof of Theorem 1. Let $f: D^{\hat{E}} \rightarrow \mathbb{R}$ be a function obtained by taking the sum of the following functions. Each variable $u v \in \hat{E}$ here has a domain $D(u) \cup\{\perp\}$. We add the function $p_{\pi}$ on $(u v, v u)$ for each permutation constraint $\pi(\varphi(u))=\varphi(v)$ of $C$. We add the function $t_{a, b}$ on $(u v, v u)$ for each two-fan constraint $(\varphi(u)=a) \vee(\varphi(v)=b)$ of $C$. Meanwhile, we add the $d$-ary function $e$ on $\delta(v)$ for each variable $v \in V \backslash A$, where $d=|\delta(v)|$ is the degree of $v$. Similarly for each variable $v \in A$, we add the $(d+1)$-ary function $e$ on $\delta(v)$ with one argument fixed to $\varphi(v)$ (hence it acts as a $d$-ary function), where $d=|\delta(v)|$. Then, from Lemma $27, f$ is $k$-submodular.

We first observe that we can convert an input $\boldsymbol{b} \in D^{\hat{E}}$ of $f$ with $f(\boldsymbol{b})<\infty$ to a half-integral $\mathcal{F}_{C, \varphi_{A}}$-cover $x$ with $x(V)=f(\boldsymbol{b})$ and convert a half-integral $\mathcal{F}_{C, \varphi_{A}}$-cover $x$ to an input $\boldsymbol{b} \in D^{\hat{E}}$ 
with $f(\boldsymbol{b}) \leq x(V)$. For each vertex $v$, we denote by $f_{v}(\boldsymbol{b})$ the value of the function $e$ for $v$, that is, for $v \in V \backslash A, f_{v}(\boldsymbol{b}):=e\left(\left.\boldsymbol{b}\right|_{\delta(v)}\right)$, where $\left.\boldsymbol{b}\right|_{\delta(v)}$ is the restriction of $\boldsymbol{b}$ to $\delta(v)$, and for $v \in A$, $f_{v}(\boldsymbol{b}):=e\left(\left.\boldsymbol{b}\right|_{\delta(v)}, \varphi_{A}(v)\right)$. We have $f(\boldsymbol{b})=\sum_{v \in V} f_{v}(\boldsymbol{b})$ when $f(\boldsymbol{b})<\infty$.

Let $\boldsymbol{b} \in D^{\hat{E}}$ be an input of $f$ with $f(\boldsymbol{b})<\infty$. We construct $x$ by setting $x(v)=f_{v}(\boldsymbol{b})$ for each $v \in V$. To see that $x$ is a (half-integral) $\mathcal{F}_{C, \varphi_{A}}$-cover, take a $\varphi_{A}$-conflicting walk $W=\left(v_{0}, \ldots, v_{\ell}\right)$ with $v_{0}, v_{\ell} \in A$. Now, consider a sequence of variables for $f$ along the walk $W$ :

$$
v_{0} v_{1}, v_{1} v_{0}, v_{1} v_{2}, v_{2} v_{1}, \ldots, v_{\ell-2} v_{\ell-1}, v_{\ell-1} v_{\ell-2}, v_{\ell-1} v_{\ell}, v_{\ell} v_{\ell-1}
$$

Let $b_{v_{0} v_{-1}}=\varphi_{A}\left(v_{0}\right)$ and $b_{v_{\ell} v_{\ell+1}}=\varphi_{A}\left(v_{\ell}\right)$. As the walk $P$ is $\varphi_{A}$-conflicting, we must have an index $0 \leq i \leq \ell$ such that $\perp \neq b_{v_{i} v_{i-1}} \neq b_{v_{i} v_{i+1}} \neq \perp$ or two indices $0 \leq i<j \leq \ell$ such that $b_{v_{i} v_{i-1}} \neq \perp$, $b_{v_{i} v_{i+1}}=\perp, b_{v_{j-1} v_{j}}=\perp$, and $b_{v_{j} v_{j+1}} \neq \perp$. In both cases, $x(V(P)) \geq 1$.

Next, let $x$ be a half-integral $\mathcal{F}_{C, \varphi_{A}}$-cover. Let $\varphi_{R(x)}$ be the satisfying assignment for $C[R(x)]$. We then define an input $\boldsymbol{b}$ for $f$ as follows:

$$
b_{u v}= \begin{cases}\varphi_{R(x)}(u) & \text { if } u \in R(x), \\ C_{v u}\left(\varphi_{R(x)}(v)\right) & \text { if } u \notin R(x), v \in R(x), \text { and } C_{v u}\left(\varphi_{R(x)}(v)\right) \neq \text { all }, \\ \perp & \text { otherwise. }\end{cases}
$$

We have $f(\boldsymbol{b})<\infty$ from the construction. For every vertex $u \in V$ with $x(u)=0$, we have $b_{u v}=\varphi_{R(x)}(u)$ for every $u v \in \delta(u)$ if $u \in R(x)$. Otherwise, we have $b_{u v}=\perp$ for every $u v \in \delta(u)$. We have $f_{v}(\boldsymbol{b})=0$ in both cases. For any vertex $u \in V$ with $x(u)=\frac{1}{2}$, there exist no edges $u v_{1}, u v_{2} \in \delta(u)$ such that $\perp \neq b_{u v_{1}} \neq b_{u v_{2}} \neq \perp$ because, otherwise, there exists a $\varphi_{A}$-conflicting walk $W$ with $x(V(W))=x(u)=\frac{1}{2}<1$, which is a contradiction. Thus, we have $f(\boldsymbol{b}) \leq x(V)$.

Now, we prove the claim. Let $x$ be a minimum half-integral $\mathcal{F}_{C, \varphi_{A}}$-cover. We then construct a minimizer $\boldsymbol{b} \in D^{\hat{E}}$ of $f$ with $f(\boldsymbol{b})=x(V)$. From Lemma 26, there is a minimizer $\boldsymbol{a} \in D_{I}^{\hat{E}}$ of $f_{I}$ such that $a_{u v}=b_{u v}$ for every $u v \in \hat{E}$ with $b_{u v} \neq \perp$. From the construction above, this means the existence of the desired deletion set $X:=\left\{v \in V \mid f_{v}(\boldsymbol{a})=1\right\}$.

\section{B Axiomatic Model}

We introduce an equivalent formulation of conflicting/implicational walks. The merit of this formulation is that checking whether a set of walks satisfies the conditions below is often easier than finding an explicit expression as 0/1/all constraints.

Definition 6. A pair $\left(\mathcal{F}, \mathcal{F}^{*}\right)$ of (possibly infinite) sets of walks in the same graph is called nice if it satisfies the following conditions.

1. $\mathcal{F} \subseteq \mathcal{F}^{*}$.

2. $\mathcal{F}^{*}$ is closed under taking a prefix (i.e., for any walk $W \in \mathcal{F}^{*}$ and any prefix-subwalk $P$ of $W, P \in \mathcal{F}^{*}$ holds).

3. For two walks $P, Q \in \mathcal{F}^{*}$ ending at the same vertex, we write $P \equiv Q$ if and only if $P \circ Q^{-1} \notin \mathcal{F}$. The relation (三) then becomes an equivalence relation, that is, (1) $P \equiv P$, (2) $P \equiv Q \Longleftrightarrow$ $Q \equiv P$, and (3) $P \equiv Q \wedge Q \equiv R \Longrightarrow P \equiv R$ hold for every walks $P, Q, R \in \mathcal{F}^{*}$ ending at the same vertex. 
4. For any equivalent walks $P, Q \in \mathcal{F}^{*}$ ending at $u$ and any edge $u v \in E, P \circ u v \in \mathcal{F}^{*}$ if and only if $Q \circ u v \in \mathcal{F}^{*}$.

For two walks $P, Q \in \mathcal{F}^{*}$ ending at the same vertex, we write $P \not \equiv Q$ if and only if $P \circ Q^{-1} \in \mathcal{F}$. The notations $P \equiv Q$ or $P \not \equiv Q$ implicitly imply that $P, Q \in \mathcal{F}^{*}$ and $t(P)=t(Q)$. Note that, from the definition, $P \in \mathcal{F} \Longleftrightarrow P^{-1} \in \mathcal{F}$ always holds. However, $P \in \mathcal{F}^{*} \Longleftrightarrow P^{-1} \in \mathcal{F}^{*}$ may not hold. When $\mathcal{F}^{*}$ is the set of all walks starting from a set of vertices $A,\left(\mathcal{F}, \mathcal{F}^{*}\right)$ is nice if and only if $\mathcal{F}$ is the set of non-returning $A$-walks (cf. [25, pp. 109-111]).

Lemma 28. Let $\mathcal{F}^{*}$ be the set of all implicational walks and let $\mathcal{F}$ be the set of all conflicting walks. Then $\left(\mathcal{F}, \mathcal{F}^{*}\right)$ is nice.

Proof. Conditions 1, 2, and 4 are trivial from the definition, and we only prove Condition 3. From Lemma 1, for any two walks $P, Q \in \mathcal{F}^{*}$ ending at the same vertex, $P \equiv Q$ if and only if $\operatorname{imp}(P)=\operatorname{imp}(Q)$ holds. Therefore, $(\equiv)$ is an equivalence relation.

Lemma 29. For any nice pair $\left(\mathcal{F}, \mathcal{F}^{*}\right)$ for a graph $G=(V, E)$, there exist domains for $V, 0 / 1 /$ all constraints for $E$, and a partial assignment $\varphi_{A}$ for some $A \subseteq V$ such that $\mathcal{F}$ is exactly the set of all $\varphi_{A}$-conflicting walks and $\mathcal{F}^{*}$ is exactly the set of all $\varphi_{A}$-implicational walks.

Proof. For each vertex $v \in V$, let $D(v)$ be the classes of equivalent walks in $\mathcal{F}^{*}$ ending at $v$. We denote the class containing $W$ by $[W] \in D(v)$. Let $A:=\left\{v \mid(v) \in \mathcal{F}^{*}\right\}$ and $\varphi_{A}(v):=[(v)]$. For each edge $u v \in \hat{E}$, we define a subdomain $D(u v) \subseteq D(u)$ and a function $f_{u v}: D(u v) \rightarrow D(v)$ as follows.

1. For any walk $W \in \mathcal{F}^{*}$ ending at $u,[W] \in D(u v)$ if and only if $W \circ u v \in \mathcal{F}^{*}$.

2. For any walk $W \circ u v \in \mathcal{F}^{*}, f_{u v}([W])=[W \circ u v]$ holds.

Claim 11. $D(u v)$ and $f_{u v}$ are well-defined.

Proof. Let $W^{\prime} \in[W]$. We have $W^{\prime} \circ u v \in \mathcal{F}^{*}$ if $W \circ u v \in \mathcal{F}^{*}$ (by Condition 4).

Suppose that $W^{\prime} \circ u v \not \equiv W \circ u v$, which implies $W^{\prime} \not \equiv W \circ u v \circ v u$. Meanwhile, by Condition 3 -(1), $W \circ u v \equiv W \circ u v$ holds and, hence, $W \circ u v \circ v u \equiv W$. Thus, we have $W^{\prime} \not \equiv W \circ u v \circ v u \equiv W$, which is a contradiction.

Claim 12. If $f_{u v}([W]) \in D(v u)$ holds for some class $[W] \in D(u v)$, we have $D(u)=D(u v)$, $D(v)=D(v u)$, and $f_{u v}=f_{v u}^{-1}$.

Proof. We have $f_{v u}\left(f_{u v}([W])\right)=[W \circ u v \circ v u]=[W]$ because $W \circ u v \circ v u \equiv W$ holds. Suppose that there exists a class $\left[W^{\prime}\right] \in D(u) \backslash D(u v)$ (i.e., $\left.W^{\prime} \circ u v \notin \mathcal{F}^{*}\right)$.

Since $\left[W^{\prime}\right] \neq[W] \in D(u v)$, we have $W^{\prime} \not \equiv W \equiv W \circ u v \circ v u$, implying $W^{\prime} \circ u v \in \mathcal{F}^{*}$, which is a contradiction. Therefore, we have $D(u)=D(u v)$. By the same argument, we have $D(v)=D(v u)$. Thus, the claim holds.

Claim 13. If $f_{u v}([W]) \notin D(v u)$ holds for some class $[W] \in D(u v)$, there exist classes $a \in D(u)$ and $b \in D(v)$ such that $D(u v)=D(u) \backslash\{a\}, D(v u)=D(v) \backslash\{b\}, f_{u v}\left(a^{\prime}\right)=b$ for every class $a^{\prime} \in D(u v)$, and $f_{v u}\left(b^{\prime}\right)=a$ for every class $b^{\prime} \in D(v u)$. 
Proof. Let $b:=f_{u v}([W]) \notin D(v u)$. Suppose that there exists a class $\left[W^{\prime}\right] \in D(u v)$ such that $f_{u v}\left(\left[W^{\prime}\right]\right) \neq f_{u v}([W])$. Because $W \circ u v \not \equiv W^{\prime} \circ u v$ holds, we have $W \circ u v \circ v u \in \mathcal{F}^{*}$, which is a contradiction. Therefore, we have $f_{u v}\left(a^{\prime}\right)=b$ for every class $a^{\prime} \in D(u v)$. Let $[Q] \in D(v) \backslash\{b\}$. Because $Q \not \equiv W \circ u v$ holds, we have $Q \circ v u \in \mathcal{F}^{*}$. Therefore, we have $D(v u)=D(v) \backslash\{b\}$.

Pick an arbitrary class $b^{\prime} \in D(v u)$. If $a:=f_{v u}\left(b^{\prime}\right) \in D(u v)$, from Claim 12, we have $D(v u)=$ $D(v)$, which is a contradiction. Therefore, by the same argument, we have $D(u v)=D(u) \backslash\{a\}$ and $f_{v u}\left(b^{\prime}\right)=a$ for every class $b^{\prime} \in D(v u)$.

Now, we prove the lemma. We introduce a constraint for each edge $u v \in E$ as follows: Pick an arbitrary class $c \in D(u v)$. If $f_{u v}(c) \in D(v u)$ holds, from Claim 12, the edge $u v$ can be expressed as $f_{u v}(\varphi(u))=\varphi(v)$ for the permutation $f_{u v}$. If $f_{u v}(c) \notin D(v u)$ holds, from Claim 13, the edge $u v$ can be expressed as $(\varphi(u)=a) \vee(\varphi(v)=b)$ for some classes $a \in D(u)$ and $b \in D(v)$. 